\title{
Strategies for increasing the operating frequency range of vibration energy harvesters: a review
}

\author{
Dibin Zhu, Michael J Tudor and Stephen P Beeby \\ School of Electronics and Computer Science, University of Southampton, SO17 1BJ, UK \\ E-mail: dz@ecs.soton.ac.uk,mjt@ecs.soton.ac.uk and spb@ecs.soton.ac.uk
}

Received 9 December 2008, in final form 1 October 2009

Published 15 December 2009

Online at stacks.iop.org/MST/21/022001

\begin{abstract}
This review presents possible strategies to increase the operational frequency range of vibration-based micro-generators. Most vibration-based micro-generators are spring-mass-damper systems which generate maximum power when the resonant frequency of the generator matches the frequency of the ambient vibration. Any difference between these two frequencies can result in a significant decrease in generated power. This is a fundamental limitation of resonant vibration generators which restricts their capability in real applications. Possible solutions include the periodic tuning of the resonant frequency of the generator so that it matches the frequency of the ambient vibration at all times or widening the bandwidth of the generator. Periodic tuning can be achieved using mechanical or electrical methods. Bandwidth widening can be achieved using a generator array, a mechanical stopper, nonlinear (e.g. magnetic) springs or bi-stable structures. Tuning methods can be classified into intermittent tuning (power is consumed periodically to tune the device) and continuous tuning (the tuning mechanism is continuously powered). This review presents a comprehensive review of the principles and operating strategies for increasing the operating frequency range of vibration-based micro-generators presented in the literature to date. The advantages and disadvantages of each strategy are evaluated and conclusions are drawn regarding the relevant merits of each approach.
\end{abstract}

Keywords: tuning strategy review, vibration energy harvesting, wider frequency range

(Some figures in this article are in colour only in the electronic version)

\section{Introduction}

Energy harvesting is the conversion of ambient energy present in the environment into electrical energy. The primary interest in the technology derives from its capability to act as an independent power supply for wireless self-powered microsystems, as an alternative to, or to augment the use of batteries. Vibration energy harvesting converts kinetic energy present in the environment and is just one form of energy harvesting [1]. Electrical energy can also be locally generated using photovoltaic or thermoelectric effects provided there is sufficient incident light or temperature gradient, respectively. Vibration energy harvesting is particularly well suited to industrial condition monitoring applications where sensors may be embedded in machines or located in dark or dirty environments. Other kinetic energy harvesting applications include human-powered devices where a range of devices have been developed to generate electrical power from foot-strikes, knee bends and backpacks [2-4].

In practical machine-based applications, vibration levels can be very low $\left(<1 \mathrm{~m} \mathrm{~s}^{-2}\right)$ at frequencies that often correspond to the frequency of the mains electricity powering 
the plant (e.g. 50 or $60 \mathrm{~Hz}$ or harmonics). Such low levels of vibration equate to amplitudes of vibration that are in the order of a few microns and the only way to extract mechanical energy in this case is to use an inertial generator that resonates at a characteristic frequency. The limitation to this approach is that the generator is, by definition, designed to work at a single frequency. A high Q-resonance means very limited practical bandwidths over which energy can be harvested. The purpose of this review is to provide an overview of existing strategies used to increase the frequency range of vibration energy harvesters thus addressing the aforementioned limitation. To date, there are, in general, two approaches to solving this problem.

The first is to adjust, or tune, the resonant frequency of a single generator so that it matches the frequency of the ambient vibration at all times. This can be achieved by changing the mechanical characteristics of the structure or electrical load on the generator. Changing the mechanical characteristics of a generator has previously been called passive or active tuning depending on the approach [5]. Passive tuning is defined as a tuning mechanism that operates periodically. This approach only consumes power during the tuning operation and uses negligible energy once the generator is matched to the frequency of the ambient vibrations. Active tuning is defined as a tuning mechanism that is continuously applied even if the resonant frequency equals the ambient vibration frequency. Since both of these approaches involve some form of active process, we will use a more precise classification scheme defining tuning mechanisms as either intermittent (previously called passive) or continuous (previously called active) throughout this review.

The second approach is to widen the bandwidth of the generator. This can be achieved by, for example, employing the following:

- an array of structures each with a different resonant frequency,

- an amplitude limiter,

- coupled oscillators,

- nonlinear (e.g. magnetic) springs,

- bi-stable structures,

- a large inertial mass (large device size) with a high degree of damping.

This review presents vibration-powered generators that demonstrate these approaches as well as those reported frequency tuning strategies for other resonant devices that are potentially applicable for tuning vibration-based microgenerators. The following section describes the theory behind tuning strategies, compares the power consumption for continuous and intermittent tuning mechanisms and suggests criteria for evaluating tuning mechanisms. Section 3 introduces the potential mechanical parameters that can be adjusted to achieve frequency tuning and reviews the approaches presented in the literature to date. Section 4 discusses the theory of electrical tuning for piezoelectric generators and presents example devices. Section 5 contains examples of wide-bandwidth generators that demonstrate the principles introduced above. Section 6 gives a tabulated

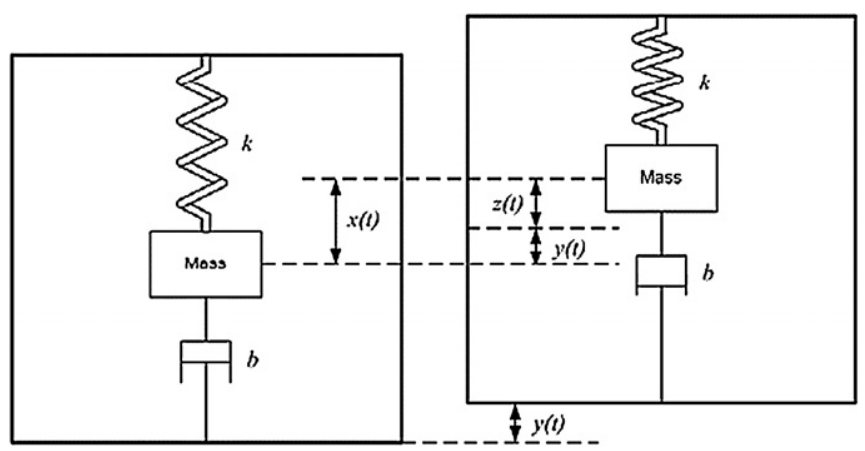

Figure 1. Generic model of a vibration-based micro-generator.

summary of devices presented to date categorized into three approaches: continuous tuning, intermittent tuning and generator arrays. Section 7 compares tuning strategies and presents theoretical power output versus frequency graphs for a large-size wide-bandwidth device, a generator array and a single tunable generator. This analysis considers four scenarios enabling a broad comparison between approaches.

\section{Strategies to tune the resonant frequency}

\subsection{Basic model of a second-order spring mass system}

Most vibration energy harvesters (also called vibration-based micro-generators) are modelled as single degree-of-freedom second-order spring-mass systems (see figure 1) as first described by Williams and Yates [6]. The system consists of an inertial frame that transmits the vibration to a suspended inertial mass, $m$, via the spring, $k$, and the damper, $b$, to produce a relative displacement or cause mechanical strain.

Transduction mechanisms such as electromagnetic [7], piezoelectric [8] and electrostatic [9] can be employed to generate electrical energy by exploiting the relative displacement or strain.

For high efficiency, the generator has to be designed to maximize the coupling between the mechanical energy source and the transduction mechanism. Mathematical analysis [6] gives the net power available within the generator as

$$
P=\frac{m \zeta_{T} Y^{2}\left(\frac{\omega}{\omega_{r}}\right)^{3} \omega^{3}}{\left[1-\left(\frac{\omega}{\omega_{r}}\right)^{2}\right]^{2}+\left[2 \zeta_{T} \frac{\omega}{\omega_{r}}\right]^{2}}
$$

where $m$ is the inertial mass and $\zeta_{T}$ is the total damping factor. $Y$ is the maximum amplitude of vibration and $\omega$ is the angular frequency of vibration. $\omega_{r}$ is the resonant frequency of the generator.

Figure 2 shows the power spectrum of the generator with various damping factors and Q-factors; the maximum power is generated when the resonant frequency of the generator matches the frequency of the ambient vibration. As generators are usually designed to have a high Q-factor to maximize harvested energy, the generated power drops dramatically if these two frequencies do not match. Most reported generators are designed to work only at one particular frequency [1]. In applications such as moving vehicles, human movement and wind-induced vibration where the frequency 


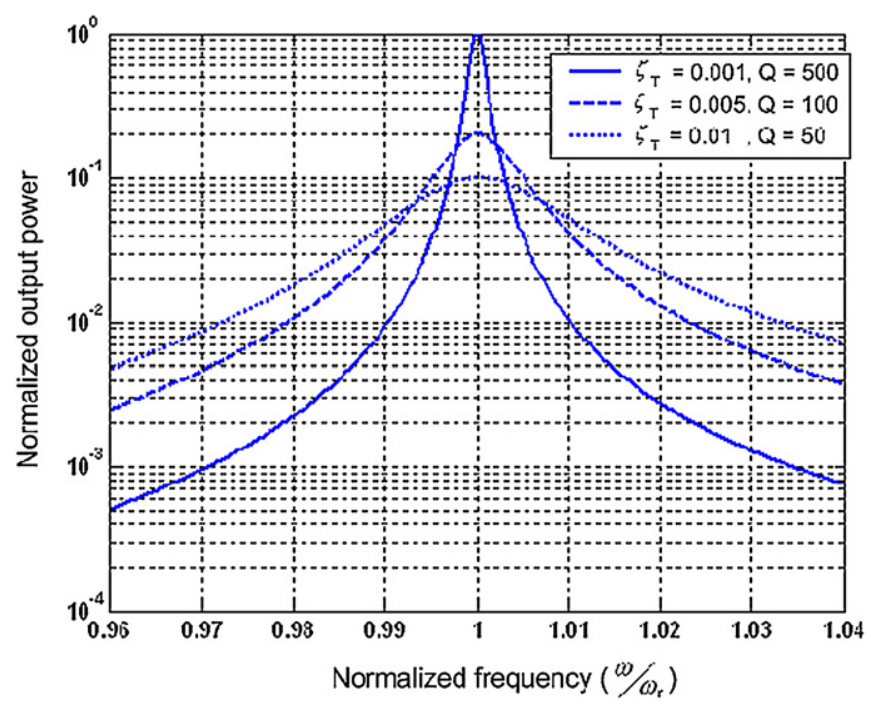

Figure 2. Power spectrum of a generator with various damping factors and Q-factors.

of ambient vibration changes periodically, the efficiency of generators with one fixed resonant frequency is significantly reduced since the generator will not always be at resonance. This limitation must be overcome if vibration-based microgenerators are to be widely applicable in powering wireless systems.

\subsection{Intermittent versus continuous tuning}

As described in the introduction, continuous tuning is applied constantly to the generator and therefore consumes more energy than intermittent tuning. It was concluded by Roundy [5] that generators using a continuous tuning mechanism can never produce a net increase in the power output as the power required to tune the resonant frequency will always exceed the increase in output power resulting from the frequency tuning. However, the derivation presented was not correct as is shown below in equations (12)-(16). For clarity the derivation in [5] is reproduced here in equations (2)-(11).

The analysis covers resonant frequency tuning achieved by providing an additional force proportional to the generator's displacement (i.e. altering stiffness) or acceleration (i.e. altering mass) using a tuning actuator [5].

The power required by the tuning actuator, $P_{a}(t)$, is

$$
P_{a}(t)=k_{a} z(t) \frac{\mathrm{d} z(t)}{\mathrm{d} t}=m_{a} \frac{\mathrm{d} z(t)}{\mathrm{d} t} \cdot \frac{\mathrm{d}^{2} z(t)}{\mathrm{d} t^{2}}
$$

where $k_{a}$ is the actuator stiffness, $z(t)$ is the displacement of the proof mass and $m_{a}$ is the mass of the actuator. The untuned resonant frequency, $\omega_{1}$, and the new resonant frequency, $\omega_{2}$, are given by

$$
\begin{gathered}
\omega_{1}=\sqrt{\frac{k}{m}} \\
\omega_{2}=\sqrt{\frac{k+k_{a}}{m} .}
\end{gathered}
$$

The actuator stiffness, $k_{a}$, can be represented as

$$
k_{a}=m \omega_{2}^{2}-k=m\left(\omega_{2}^{2}-\omega_{1}^{2}\right) .
$$

Hence, (2) can be written as

$$
P_{a}(t)=\frac{1}{2} m \omega_{2}\left(\omega_{2}^{2}-\omega_{1}^{2}\right) Z^{2} \sin \left(2 \omega_{2} t\right) .
$$

Therefore, the average power of the actuation is

$$
\begin{aligned}
\left|P_{a}\right| & =\frac{1}{T} \int_{0}^{T} P_{a}(t) \mathrm{d} t \\
& =\frac{1}{T} \int_{0}^{T}\left[\frac{1}{2} m \omega_{2}\left(\omega_{2}^{2}-\omega_{1}^{2}\right) Z^{2} \sin \left(2 \omega_{2} t\right)\right] \mathrm{d} t=0 .
\end{aligned}
$$

In practice, actuation power can never be zero if the tuning mechanism is activated. Roundy et al used the maximum power rather than the average power of actuation in their derivation and drew the conclusion that the active tuning can never produce net increase of output power. In addition, only the situation where the tuning force is proportional to the generator's displacement or acceleration was studied in [5]. For most cases, the tuning force is not linked to the generator's movement and methods of calculating actuation power may vary according to the way in which the tuning force is applied. Therefore, the analysis in [5] does not apply to all situations.

However, it is apparent that intermittent tuning has an advantage over a continuous tuning mechanism because it is switched off once the device is at resonance and therefore consumes less energy than continuous mechanisms.

\subsection{Evaluating tuning approaches}

The suitability of different tuning approaches will depend upon the application, but in general terms the key factors for evaluating a tuning mechanism for adjusting the resonant frequency of vibration-based micro-generators are as follows.

- The energy consumed by the tuning mechanism should be as small as possible and must not exceed the energy produced by the generator.

- The mechanism should achieve a sufficient operational frequency range.

- The tuning mechanism should achieve a suitable degree of frequency resolution.

- The generator should have as high as possible Q-factor to achieve the maximum power output and the strategy applied should not increase the damping, i.e. decrease Q-factor, over the entire operational frequency range.

A generator's resonant frequency can be tuned by both mechanical and electrical methods. Mechanical tuning alters the resonant frequency by changing mechanical properties of the structure. Electrical tuning alters the resonant frequency by adjusting the electrical load. The principles of both methods as well as existing approaches to realize them are described in the following sections.

\section{Mechanical tuning methods}

As most reported vibration energy-harvesting devices are based on a cantilever [1], we will use this structure in the following theoretical analyses of mechanical tuning. The principles demonstrated are, however, generally applicable to all mechanical resonator structures. This section covers mechanisms that achieve tuning by 


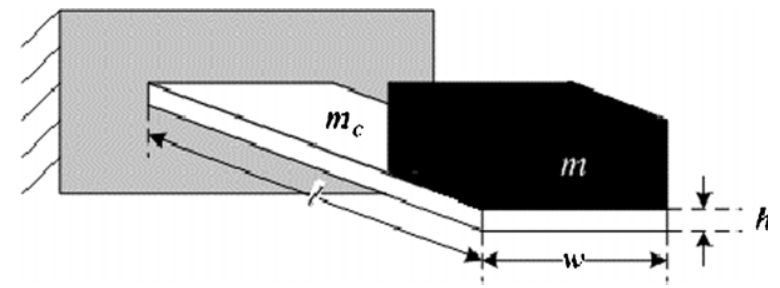

Figure 3. Cantilever with a mass.

- changing dimensions,

- moving the centre of gravity of the proof mass,

- variable spring stiffness,

- straining the structure.

A comprehensive review of each mechanical tuning mechanism reported in the literature to date is presented after a brief analysis of the theory.

The resonant frequency of a spring-mass structure is given by

$$
f_{r}=\frac{1}{2 \pi} \sqrt{\frac{k}{m}}
$$

where $k$ is the spring constant and $m$ is the inertial mass. When tuning the resonant frequency of the generator, one can change either the spring constant or the mass.

The spring constant of a resonator depends on its materials and dimensions. For a cantilever with a mass at the free end (figure 3), the resonant frequency is given by [10]

$$
f_{r}=\frac{1}{2 \pi} \sqrt{\frac{Y w h^{3}}{4 l^{3}\left(m+0.24 m_{c}\right)}}
$$

where $Y$ is Young's modulus of the cantilever material; $w$, $h$ and $l$ are the width, thickness and length of the cantilever, respectively, and $m_{c}$ is the mass of the cantilever. The resonant frequency can be tuned by adjusting all these parameters. Each parameter in equation (9) is discussed in the following sections.

In addition, it is important to mention that if actuators are involved in changing the mechanical properties of the resonant structure, the tuning mechanisms can be operated by a control system to automatically tune the generator.

\subsection{Changing dimensions}

In practice, it is difficult to change the width, $w$, and thickness, $h$, of a cantilever while changing the length, $l$, is feasible. Furthermore, modifying $l$ is suitable for intermittent tuning. The approach requires that the cantilever base clamp be released and reclamped in a new location along the length of the beam thereby changing the effective length (and hence frequency). There is no power required to maintain the new resonant frequency. Furthermore, as the resonant frequency is inversely proportional to $l^{3 / 2}$, thus modifying $l$ can significantly change $f_{r}$.

Suppose $l$ is the original length of the cantilever and $l^{\prime}$ is the modified length of the cantilever, $l^{\prime}=l+\Delta$, where $\Delta$ is the difference between them. The mass of a cantilever is then changed to $m_{c}{ }^{\prime}=w h l^{\prime} \rho$, where $\rho$ is the density of the

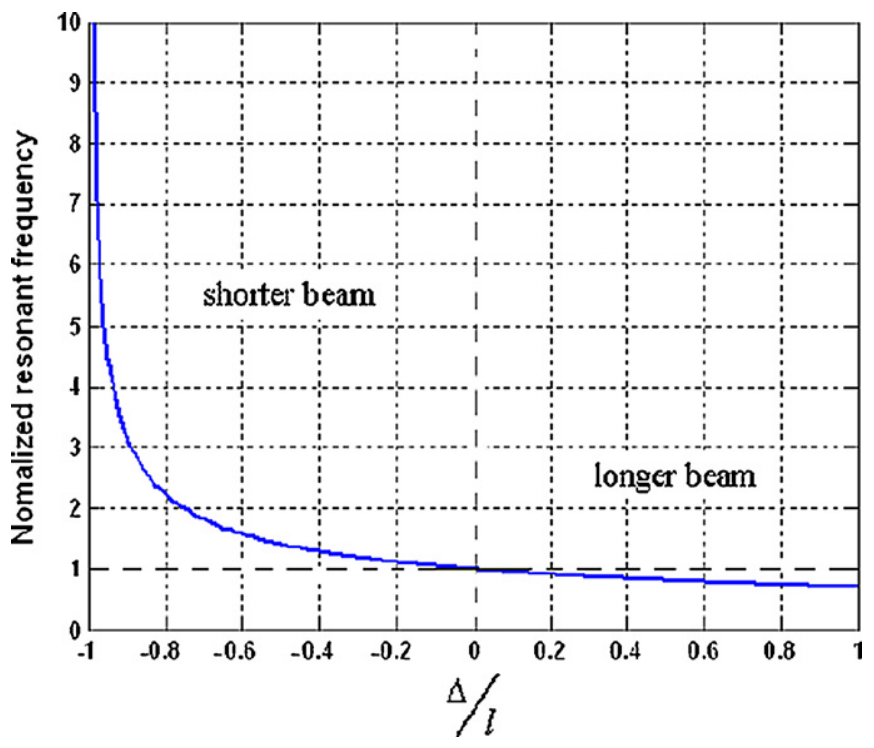

Figure 4. Normalized resonant frequency with a variation of cantilever length.

cantilever material while the original mass of a cantilever is $m_{c}=w h l \rho$. Then the new resonant frequency becomes

$$
\begin{aligned}
f_{r}^{\prime} & =\frac{1}{2 \pi} \sqrt{\frac{Y w h^{3}}{4 l^{\prime 3}\left(m+0.24 m_{c}^{\prime}\right)}} \\
& =\frac{1}{2 \pi} \sqrt{\frac{Y w h^{3}}{4(l+\Delta)^{3}\{m+0.24[w h(l+\Delta)] \rho\}}} .
\end{aligned}
$$

And the ratio of the tuned frequency to the original frequency called the normalized resonant frequency is

$$
\begin{aligned}
\frac{f_{r}^{\prime}}{f_{r}} & =\sqrt{\frac{l^{3}\left(m+0.24 m_{c}\right)}{l^{3}\left(m+0.24 m_{c}^{\prime}\right)}} \\
& =\sqrt{\frac{l^{3}(m+0.24 w h l \rho)}{(l+\Delta)^{3}\{m+0.24[w h(l+\Delta)] \rho\}}} .
\end{aligned}
$$

Figure 5 shows the normalized resonant frequency with the variation of cantilever length where a negative $\Delta / l$ means that the new cantilever beam is shorter than its original length and thus has a higher resonant frequency. A positive $\Delta / l$ means that the cantilever beam has been lengthened giving a lower resonant frequency. Figure 4 shows that it is more efficient to tune the resonant frequency by shortening the cantilever beam.

An example of this approach is described in a patent by Gieras et al [11]. Figure 5 shows the side view of the proposed device. The electromagnetic generator consists of a cantilever with a set of magnets fixed to its free end. The cantilever is clamped to a base using screws. A coil is placed between the magnets to pick up output power. A slider is connected to a linear actuator which moves the slider back and forth to adjust the effective length of the cantilever, $L$, and hence the resonant frequency of the generator.

\subsection{Moving centre of gravity of the proof mass}

It is difficult to add or remove a mass after a generator has been fabricated. However, the resonant frequency of a cantilever 


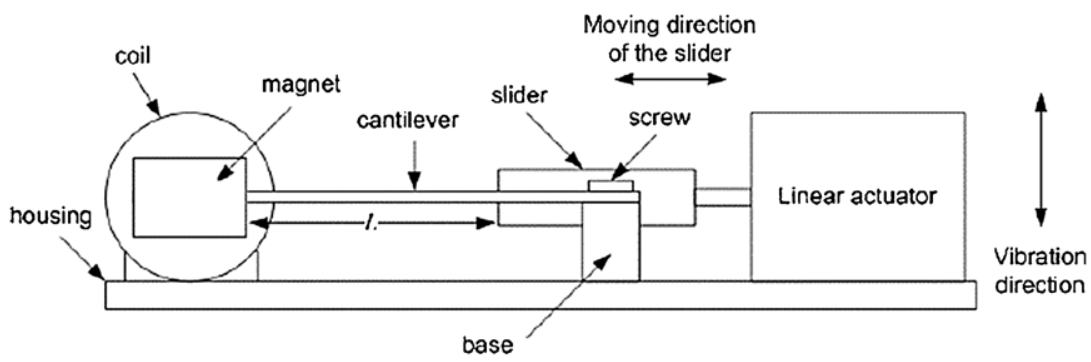

Figure 5. Side view of a proposed self-adjustable energy-harvesting system.

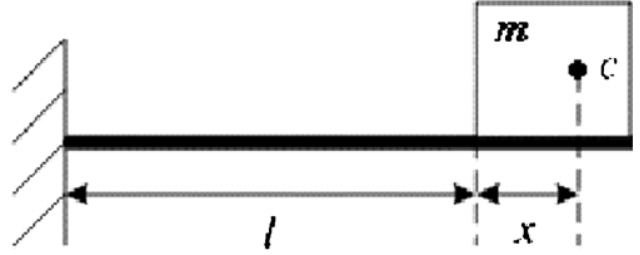

Figure 6. Side view of a cantilever structure.

structure can be adjusted by moving the centre of gravity of the inertial mass. Figure 6 shows the side view of a cantilever with a mass on the free end.

The length of the cantilever without the mass is $l$ and the proof mass on its free end is $m$. The centre of gravity of the proof mass is located at $c$ and the distance between $c$ and the end of the cantilever is $x$. The tuned resonant frequency of this structure can be approximated as [12]

$$
f_{r}^{\prime}=\frac{1}{2 \pi} \sqrt{\frac{Y w h^{3}}{12 m l^{3}} \cdot \frac{r^{2}+6 r+2}{8 r^{4}+14 r^{3}+\frac{21}{2} r^{2}+4 r+\frac{2}{3}}}
$$

where $w$ and $h$ are the width and thickness of the cantilever, respectively, and $r=\frac{x}{l}$.

Equation (9) for the resonant frequency of a cantileverbased generator, considering that the mass of the cantilever beam is negligible compared to the proof mass, can be rewritten as

$$
f_{r}=\frac{1}{2 \pi} \sqrt{\frac{Y w h^{3}}{4 l^{3} m}} .
$$

Hence, the ratio of the tuned frequency to the original frequency is

$$
\frac{f_{r}^{\prime}}{f_{r}}=\sqrt{\frac{1}{3} \cdot \frac{r^{2}+6 r+2}{8 r^{4}+14 r^{3}+\frac{21}{2} r^{2}+4 r+\frac{2}{3}}} .
$$

Figure 7 shows the normalized resonant frequency with variation of the position of the centre of gravity of the proof mass. The further the centre of gravity of the proof mass is from the end of the cantilever, the lower the resonant frequency.

$\mathrm{Wu}$ et al [13] reported a piezoelectric generator using this principle as shown in figure 8 . The proof mass of this device consisted of two parts: a fixed mass was attached to the cantilever and the other part was a movable screw. The position of the centre of gravity of the proof mass could be adjusted by changing the position of the movable screw. A fastening stud was used to fix the screw when tuning was

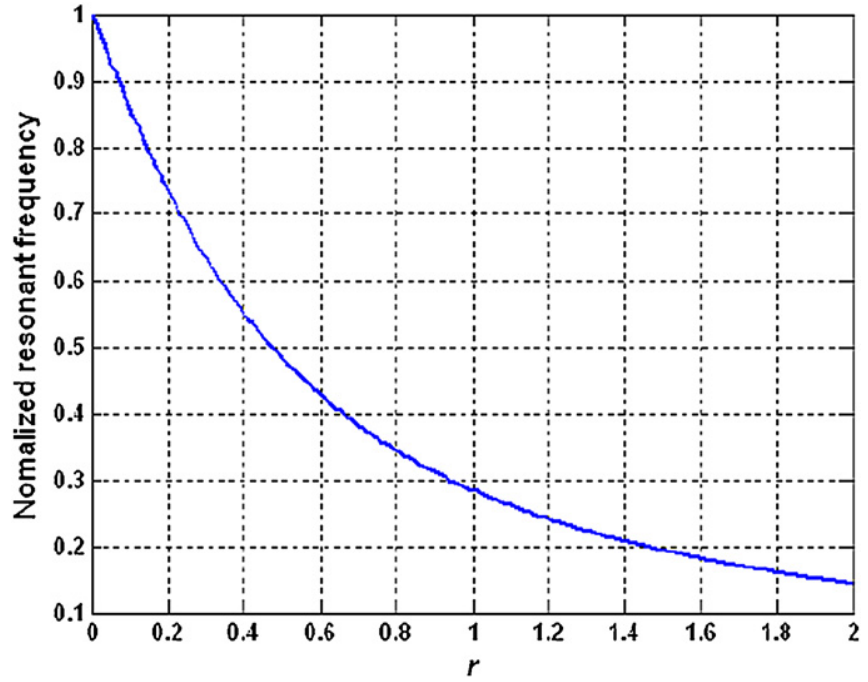

Figure 7. Normalized resonant frequency with a variation of centre of gravity positions.

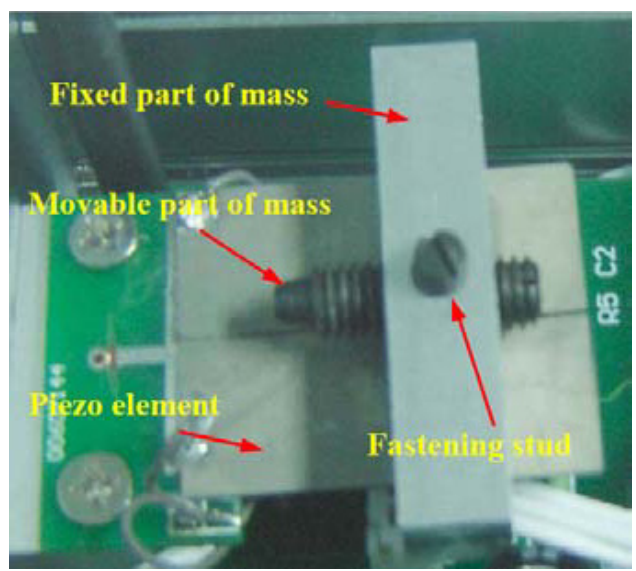

Figure 8. Picture of the piezoelectric cantilever prototype with a movable mass [13].

finished. The size of the fixed mass is $10 \mathrm{~mm} \times 12 \mathrm{~mm} \times 38$ $\mathrm{mm}$ and the movable mass is an M6 screw of length of $30 \mathrm{~mm}$. The resonant frequency of the device was tuned from $180 \mathrm{~Hz}$ to $130 \mathrm{~Hz}$ by moving the screw from one end to the other end (figure 9). The output voltage dropped with increasing resonant frequency.

This approach is suitable for fine frequency tuning of the generator before installation if the vibration frequency in the working environment is not time varying. 


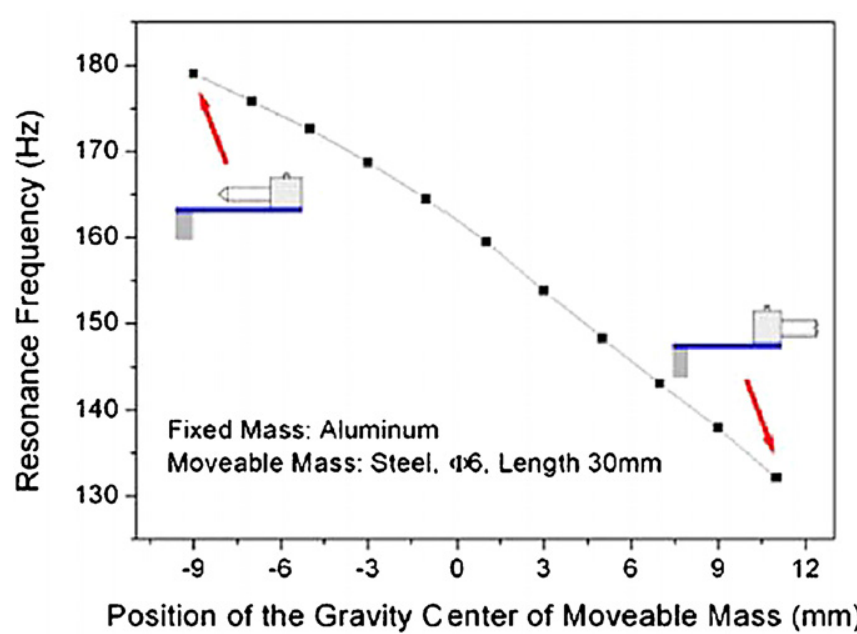

Figure 9. Experimental result of frequency adjustment [13].

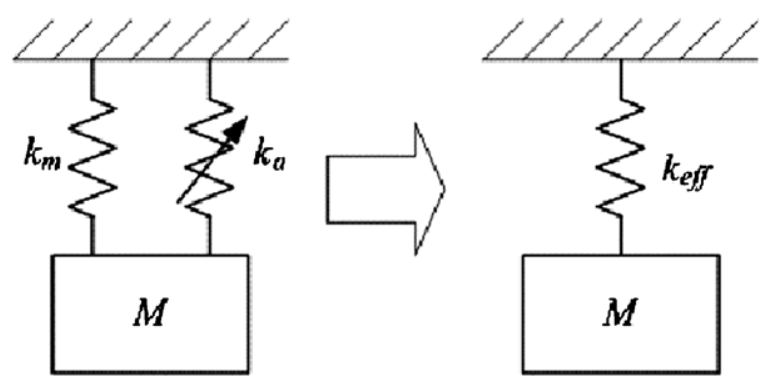

Figure 10. Model of devices with softened spring stiffness.

\subsection{Variable spring stiffness}

One commonly used method is to soften the spring stiffness. The principle is to apply a 'negative' spring in parallel to the mechanical spring. Therefore, the effective spring constant of such a device, $k_{\text {eff }}$, becomes

$$
k_{\mathrm{eff}}=k_{m}+k_{a}
$$

where $k_{m}$ is the mechanical spring constant and $k_{a}$ is an additional 'negative' spring stiffness (figure 10). The modified frequency becomes

$$
f_{r}=\frac{1}{2 \pi} \sqrt{\frac{k_{\mathrm{eff}}}{m}}=\frac{1}{2 \pi} \sqrt{\frac{k_{m}+k_{a}}{m} .}
$$

The negative spring $k_{a}$ can be applied electrostatically, piezoelectrically, magnetically or thermally. Examples of these approaches are described below. Most of these examples are tunable resonators and not energy harvesters, but the principles are identical. It is important to note, however, that the additional inertial mass present in an energy harvester (as opposed to the purely resonant structures) will reduce the tuning effectiveness and increase the power required to tune compared to the values quoted. It should also be noted that the following variable spring stiffness devices are all continuously operated expect the one on which the negative spring is applied magnetically.

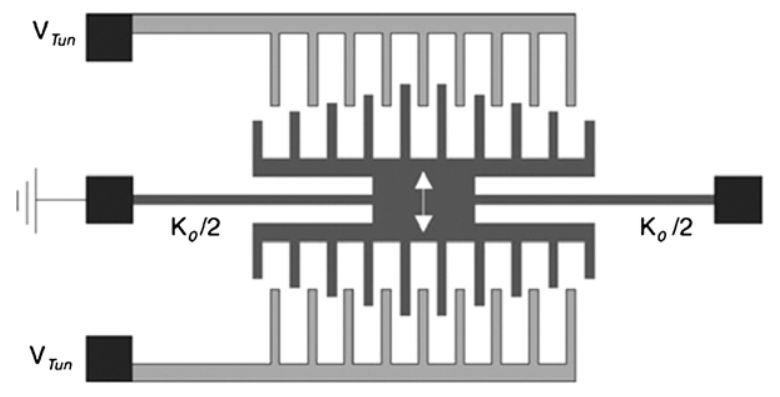

Figure 11. Resonance tuning by electrostatic softening [15].

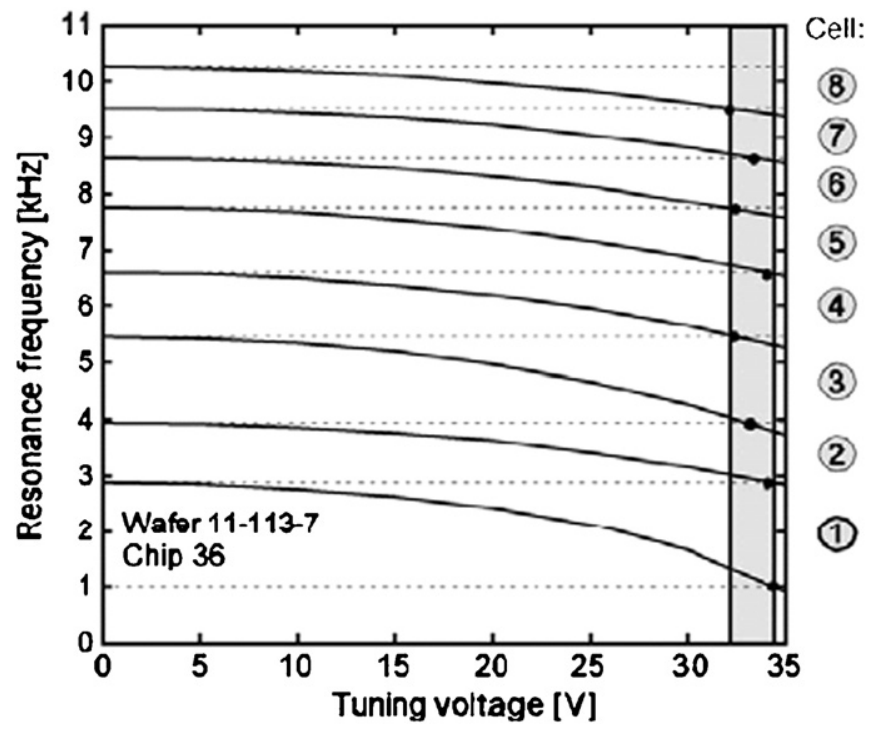

Figure 12. Resonance tuning of the array [15].

3.3.1. Electrostatic methods. Scheibner [14, 15] reported a vibration detector consisting of an array of eight comb resonators each with a different base resonant frequency. A single resonator is shown in figure 11. Each resonator comb is tuned by electrostatically softening the structure by applying a tuning voltage to the electrodes marked ' $V_{\text {Tun }}$ '. The device was designed so that the resonator array had overlapping tuning ranges which allowed continuous measurements in the frequency range of the device from $1 \mathrm{kHz}$ to $10 \mathrm{kHz}$. Figure 12 shows the tuning range of each resonator. The tuning voltage varied from $0 \mathrm{~V}$ to $35 \mathrm{~V}$. The total size of the sensor chip is $7 \mathrm{~mm} \times 10 \mathrm{~mm}$.

Adam [16] realized a tuning range from $7.7 \%$ to $146 \%$ of the central frequency of $25 \mathrm{kHz}$ of a resonator with a single comb structure (figure 13). The driving voltage ranged from $0 \mathrm{~V}$ to $50 \mathrm{~V}$ (figure 14). The total size was not mentioned in the paper but is estimated from the SEM scale to be no larger than $500 \mu \mathrm{m} \times 500 \mu \mathrm{m}$.

Lee et al [17] presented a frequency tunable comb resonator with curved comb fingers (figure 15). Fingers of the tuning comb were designed to be of curved shape to generate a constant electrostatic stiffness or linear electrostatic force that is independent of the displacement of the resonator under a control voltage. Experimentally, the resonant frequency of a laterally driven comb resonator with 186 pairs of curved 


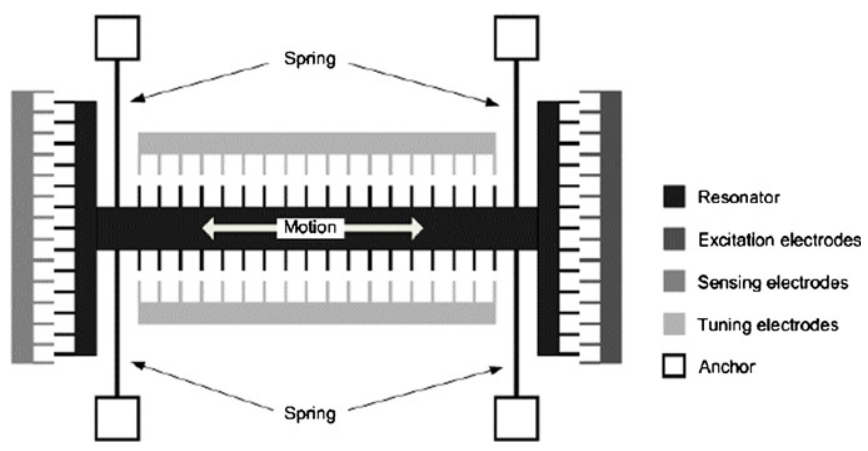

Figure 13. Schematic diagram of a single comb structure (after [16]).

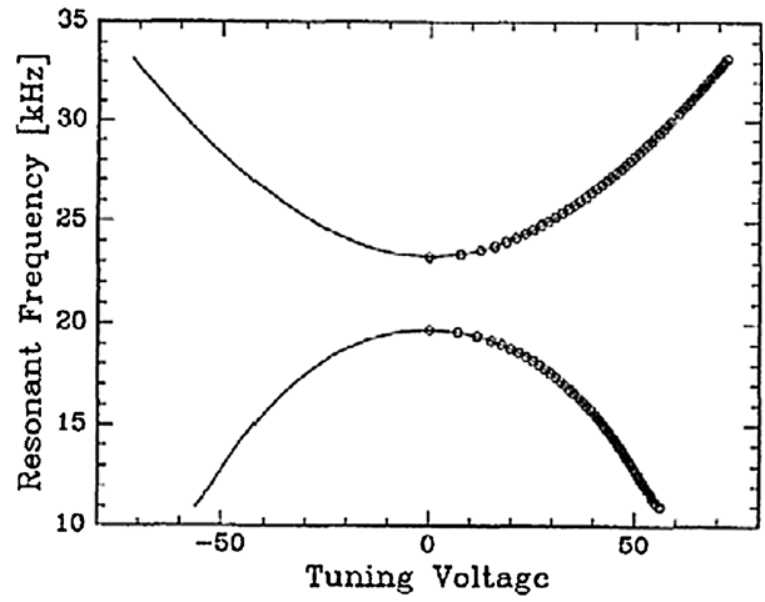

Figure 14. Resonance tuning of a single comb structure [16].

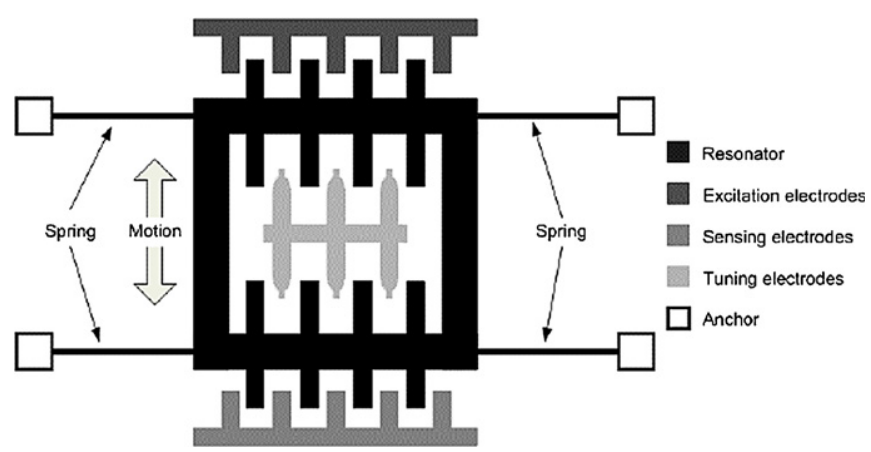

Figure 15. Schematic diagram of a comb resonator with curved tuning fingers (after [17]).

contour fingers was reduced by $55 \%$ from the initial frequency of $19 \mathrm{kHz}$ under a bias voltage of $150 \mathrm{~V}$ (figure 16). The corresponding effective stiffness was decreased by $80 \%$ from the initial value of $2.64 \mathrm{~N} \mathrm{~m}^{-1}$. The total size of the resonator is $460 \mu \mathrm{m} \times 840 \mu \mathrm{m}$. It was concluded that the closed-form approach of the comb-finger profile can be applied to other comb-shaped actuators for frequency control whilst achieving linear electrostatic stiffness with respect to displacement.

Piazza et al [18] developed a micromachined, piezoelectrically actuated and sensed, high-Q single-crystal silicon (SCS) resonator with a voltage-tunable centre frequency (figure 17). Piezoelectric transduction was

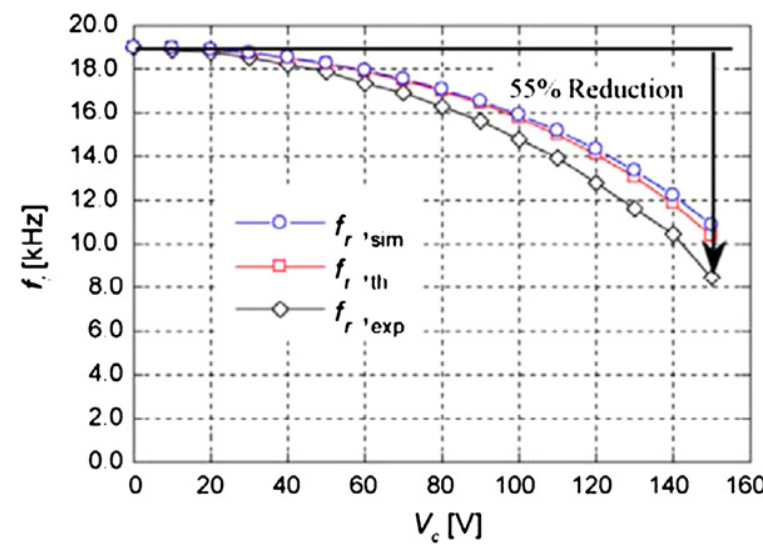

Figure 16. Resonance tuning of a comb resonator with curved tuning fingers [17].

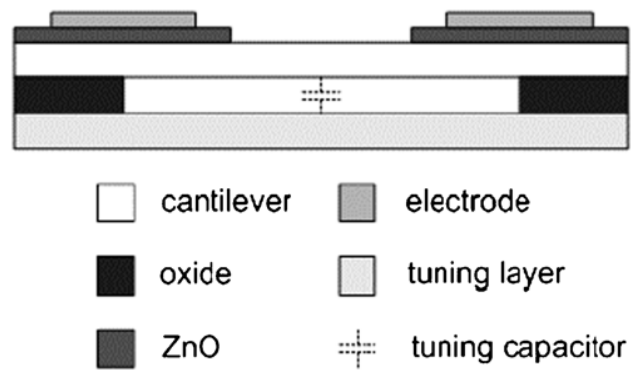

Figure 17. Voltage-tunable, piezoelectrically transduced SCS resonators: Q-enhanced configuration (after [18]).

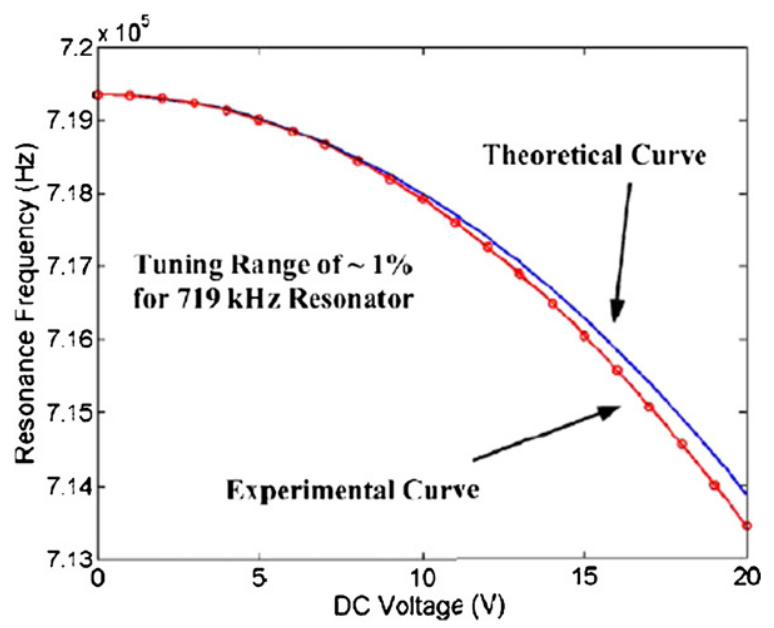

Figure 18. Electrostatic fine-tuning characteristic for a $719 \mathrm{kHz}$ piezo-resonator [18].

integrated with capacitive fine-tuning of the resonator centre frequency to compensate for any process variations. The resonant frequency could be tuned by $6 \mathrm{kHz}$ based on an untuned resonant frequency of $719 \mathrm{kHz}$ by applying an electrostatic force beneath the cantilever (figure 18). The driving voltage varied from 0 to $20 \mathrm{~V}$. The dimensions of this resonator are $200 \mu \mathrm{m} \times 20 \mu \mathrm{m} \times 4.2 \mu \mathrm{m}$.

Yao et al [19] compared frequency tuning by applying either an axial force (discussed further in section 3.4) or a transverse force on the resonator electrostatically as shown in 


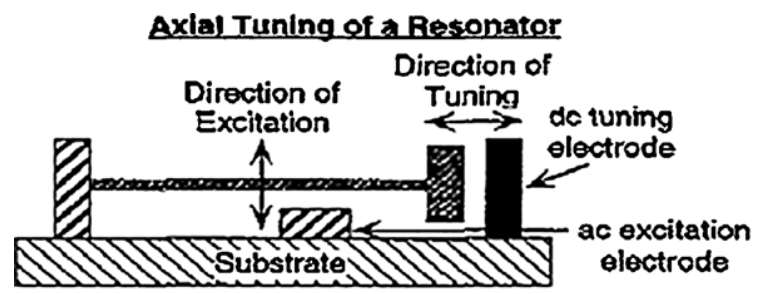

(a)

Transyerse_Tuning of a Resonator (\#-1)

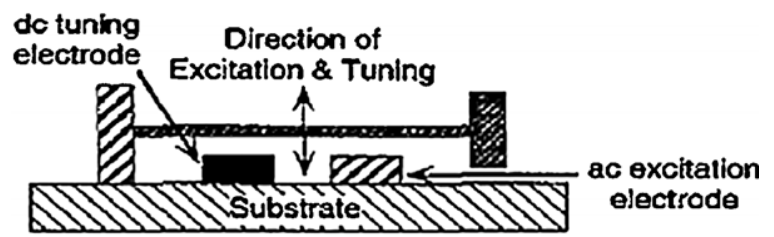

(b)

Iransverse Tuning of a Resonator (\#2)

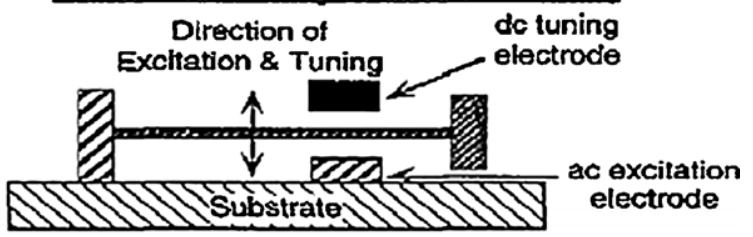

(c)

Figure 19. Schematic drawing of a simple resonator showing axial loading $(a)$, and transverse loading with the excitation and the tuning electrode on the same side $(b)$ and on the opposite side $(c)$ of the resonating rod [19].

figure 19. Frequency tuning by applying a transverse force was tested experimentally. It was found that the resonant frequency may increase or decrease with the applied tuning voltage depending on where the tuning electrode is placed with respect to the excitation electrode and the resonating rod. When the tuning electrode was placed on the same side of the excitation electrode as indicated in figure $19(b)$, the resonant frequency decreased with the increase of applied voltage. When the tuning electrode was placed on the opposite side of the excitation electrode as indicated in figure 19(c), the resonant frequency increased with the increase of applied voltage.

A micromachined resonator having an out-of-plane natural resonant frequency of $0.96 \mathrm{MHz}$ and a Q-factor of 4370 had a linear tuning range (with respect to the transverse tuning force) of $60 \mathrm{kHz}$ with a maximum required dc tuning voltage of $35 \mathrm{~V}$ (figure 20). Another resonator with an untuned resonant frequency of $149.5 \mathrm{kHz}$ was tuned to $139.5 \mathrm{kHz}$ by applying a dc tuning voltage of $30 \mathrm{~V}$ (figure 21). The actual dimensions of these devices were not mentioned. The idea was later patented by Thiesen and O'Brian in 2006 [20].

3.3.2. Piezoelectric methods. Peters et al [21] reported a tunable resonator, shown in figure 22(a), potentially suitable as a resonator structure for vibration energy harvesting. The adjustment of the resonant frequency was provided by mechanical stiffening of the structure using piezoelectric actuators. A piezoelectric actuator was used because

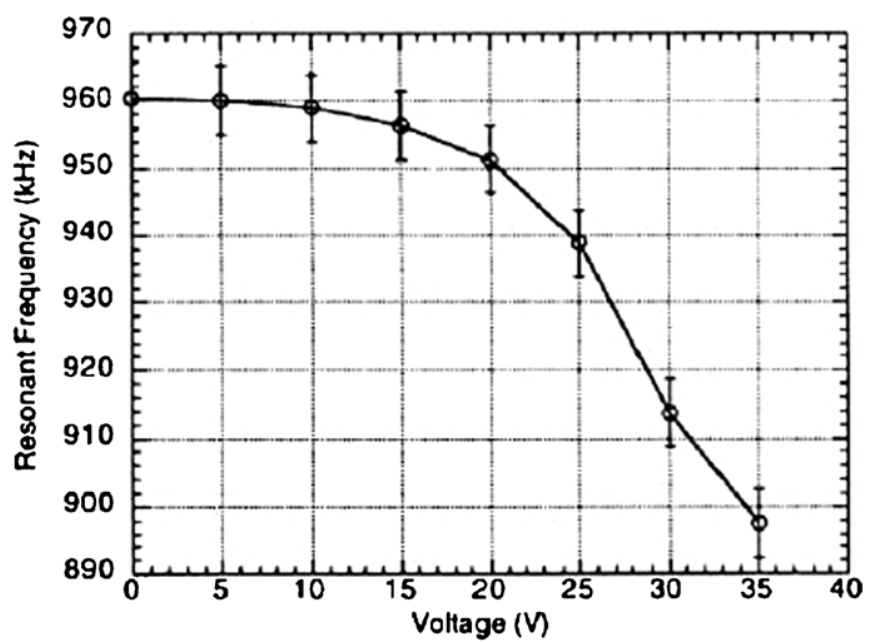

Figure 20. Measured resonant frequency versus the tuning dc voltage with an untuned resonant frequency of $0.96 \mathrm{MHz}$ (tuning mechanism as in figure 19(b)) [19].

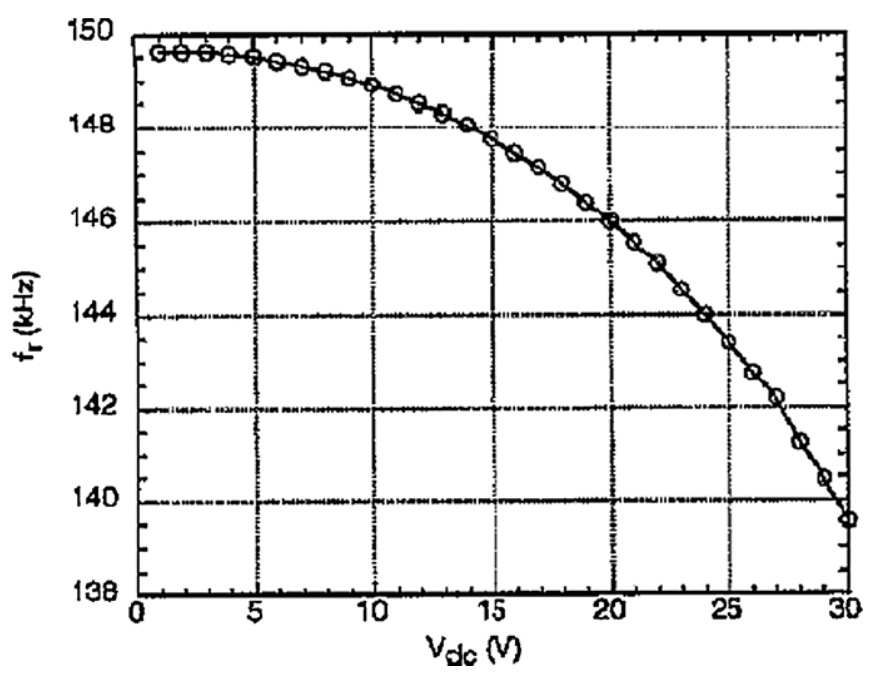

Figure 21. Measured resonant frequency versus the tuning dc voltage with an untuned resonant frequency of $1495.5 \mathrm{kHz}$ (tuning mechanism as in figure $19(b))$ [19].

piezoelectric materials can generate large forces with low power consumption. Two actuators, one clamped and one free, are connected together. The free actuator can oscillate around the axis of rotation if a suitable excitation is applied to the clamp. The stiffness of the structure was increased by applying an electrical potential to both actuators which changes the shape of the structure as shown in figure $22(c)$. Thus, the natural frequency of the rotational mass-spring system increased. The tuning voltage was chosen to be $\pm 5 \mathrm{~V}$ leading to a measured resonance shift of $\pm 15 \%$ around the initial resonant frequency of $78 \mathrm{~Hz}$, i.e. the tuning range was from $66 \mathrm{~Hz}$ to $89 \mathrm{~Hz}$ (figure 23).

3.3.3. Magnetic methods. Challa et al [22] reported an intermittently tuned piezoelectric micro-generator, $50 \mathrm{~cm}^{3}$ in volume, with a frequency range of $22-32 \mathrm{~Hz}$ based on an original resonant frequency of $26 \mathrm{~Hz}$. The tuning was realized by manually applying a magnetic force perpendicularly to 


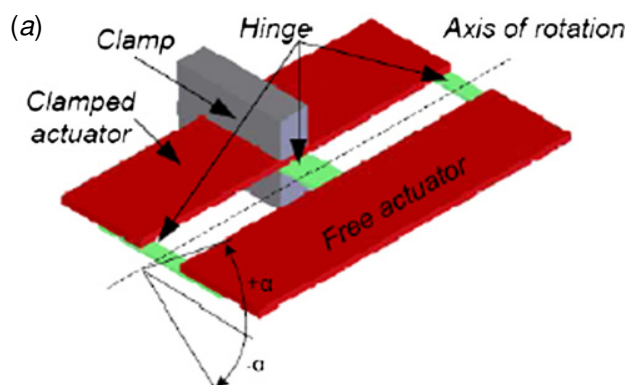

(b)

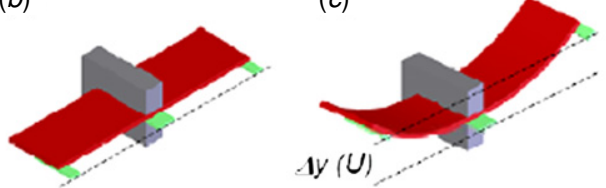

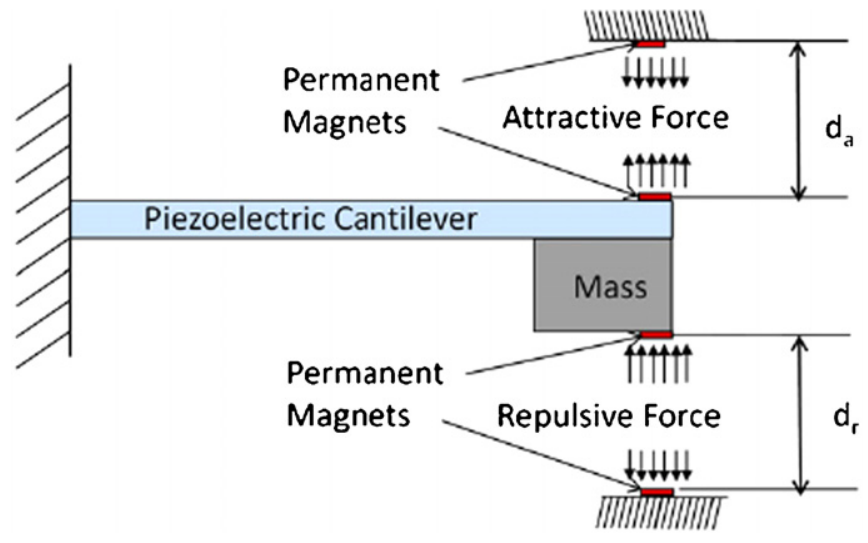

Figure 24. Schematic of the tunable piezoelectric generator [22].

Figure 22. (a) Schematic of the resonator, $(b)$ cross-section without applied voltage and $(c)$ with applied voltage [21].

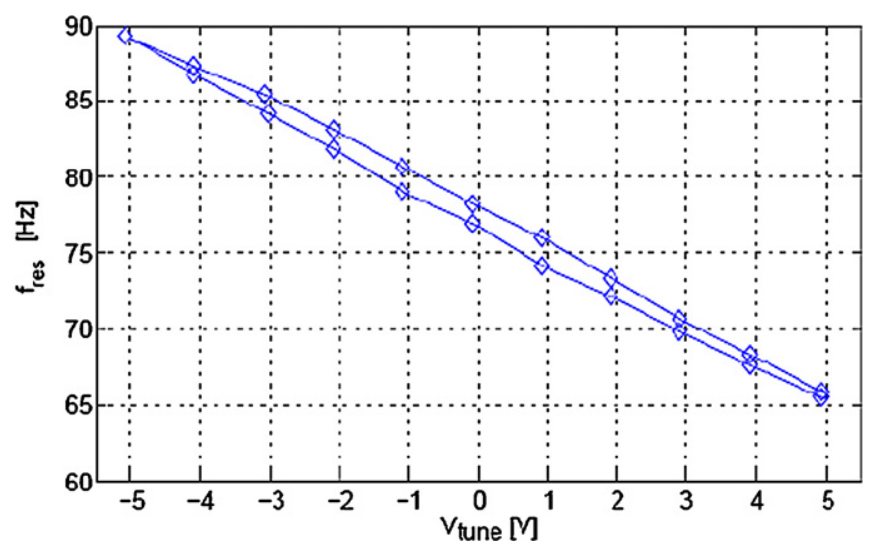

Figure 23. Measured resonant frequency versus applied tuning voltage [21].

the cantilever generator as shown in figure 24. By varying the distance between the two sets of tuning magnets on the beam and the stationary magnets, the resonant frequency of the generator can be altered. The maximum tuning distance was $3 \mathrm{~cm}$. The proposed generator produced $240-280 \mu \mathrm{W}$ power at $0.8 \mathrm{~m} \mathrm{~s}^{-2}$ acceleration, but the tuning mechanism had the unwanted side effect of varying damping over the frequency range as shown in figure 25 . The device was made of discrete components. The dimensions of the piezoelectric cantilever are $34 \mathrm{~mm} \times 20 \mathrm{~mm} \times 0.92 \mathrm{~mm}$ and the effective mass is $45.8 \mathrm{~g}$.

\subsubsection{Thermal methods. Remtema and Lin [23] used a} resistive heater to generate a thermal stress on a straight-beam spring (figure 26), which caused up to $6.5 \%$ frequency change based on a resonant frequency of $31 \mathrm{kHz}$ with a maximum temperature at $255{ }^{\circ} \mathrm{C}$. The power consumption during the process was $25 \mathrm{~mW}$. Figure 27 shows the percentage change of a resonant frequency with variation of power consumed in tuning. The size of the device is estimated to be less than $500 \mu \mathrm{m} \times 700 \mu \mathrm{m}$ from the author's description. The thermal approach is unlikely to be practical for energy harvesting since it is inherently of high power and is a continuous tuning

Syms et al [24] reported frequency tuning by applying constrained thermal expansion on a simple unfolded resonator (figure 28). The tuning range was from $-25 \%$ to $+50 \%$ with power consumption from 1.5 to $10 \mathrm{~mW}$ (figure 29). The tuning sensitivity obtained with this tuning method was $33 \%$ per $\mathrm{mW}$. It is estimated from the annotation shown in figure 28 that the device is no larger than $3000 \mu \mathrm{m} \times 3000 \mu \mathrm{m}$.

\subsection{Straining the structure}

The effective stiffness of the structure can be varied by applying a stress and therefore placing it under strain. The following theoretical analyses focus on straining a cantilever and a clamped-clamped beam. The resonant frequency of a cantilever structure can be tuned by applying an axial load. In vibration energy harvesting, most devices are based on cantilever structures especially the clamped-free (figure 30) and clamped-clamped (figure 31) cantilevers. An axial tensile load applied to a cantilever (figures 30(a) and 31(a)) increases the resonant frequency of the cantilever while an axial compressive load applied to a cantilever (figures $30(b)$ and $31(b)$ ) decreases the resonant frequency of the cantilever.

An approximate formula for the resonant frequency of a uniform cantilever in mode $i$ with an axial load, $f_{r i}$, , is given by [25]:

$$
f_{r i}^{\prime}=f_{r i} \cdot \sqrt{1+\frac{F}{F_{b}} \cdot \frac{\lambda_{1}^{2}}{\lambda_{i}^{2}}}
$$

where $f_{r}$ is the resonant frequency in mode $i$ without load, $F$ is the axial load and $F_{b}$ is the axial load required to buckle the beam, i.e. to make the fundamental resonant frequency zero. $F$ is positive for a tensile load and negative in the compressive case. Variable $\lambda_{i}$ is a dimensionless load parameter which is a function of the beam boundary conditions applied to the cantilever for the $i$ th mode of the beam. It is given by the $i$ th positive solution of equation (18) for a cantilever and of equation (19) for a clamped-clamped beam [26]:

$$
\begin{aligned}
\cos \lambda \cdot \cosh \lambda+1 & =0 \\
\cos \lambda \cdot \cosh \lambda-1 & =0 .
\end{aligned}
$$
mechanism. 


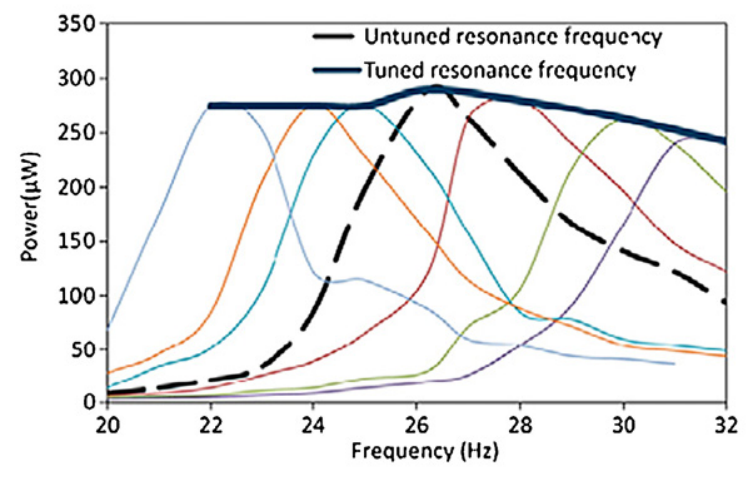

(a)

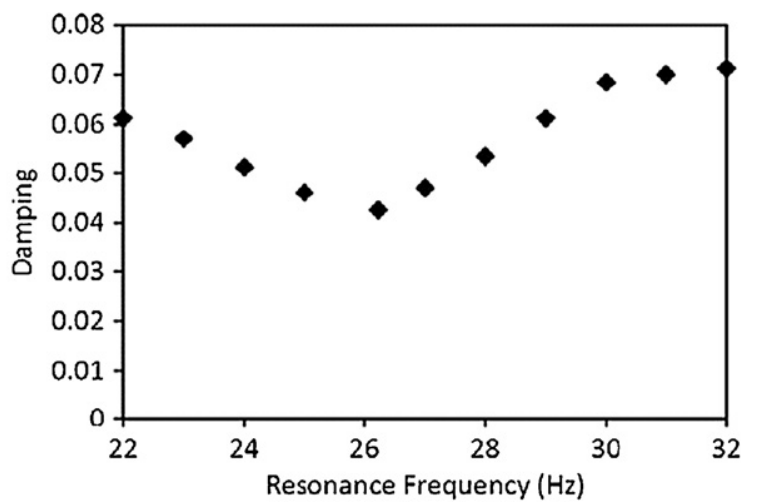

(b)

Figure 25. Output power $(a)$ and damping $(b)$ versus resonant frequency [22].

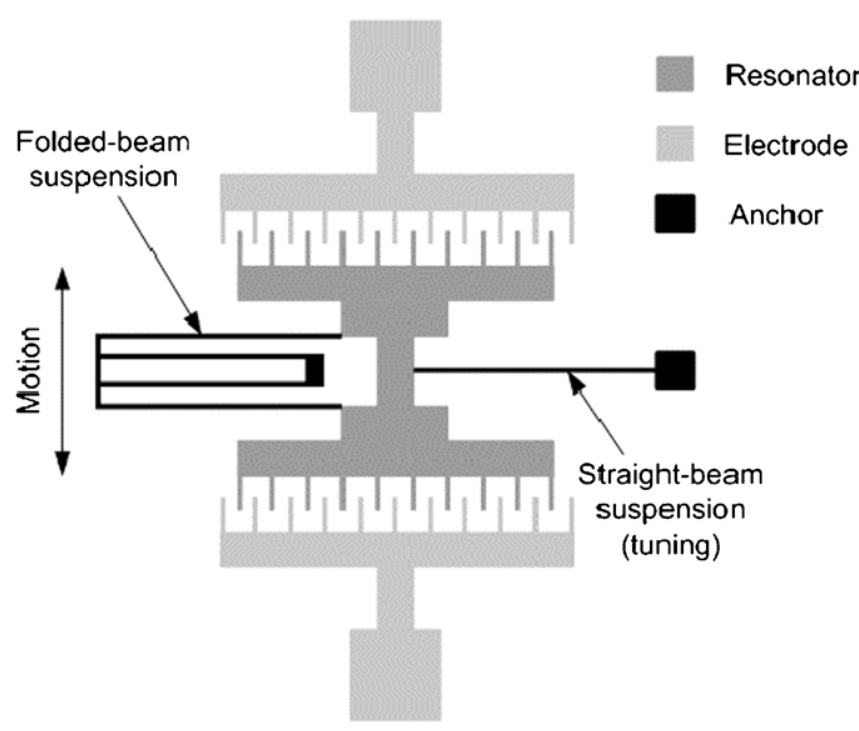

Figure 26. Schematic diagram of a comb-shape micro resonator with a straight-beam for active frequency tuning via localized stressing effects (after [23]).

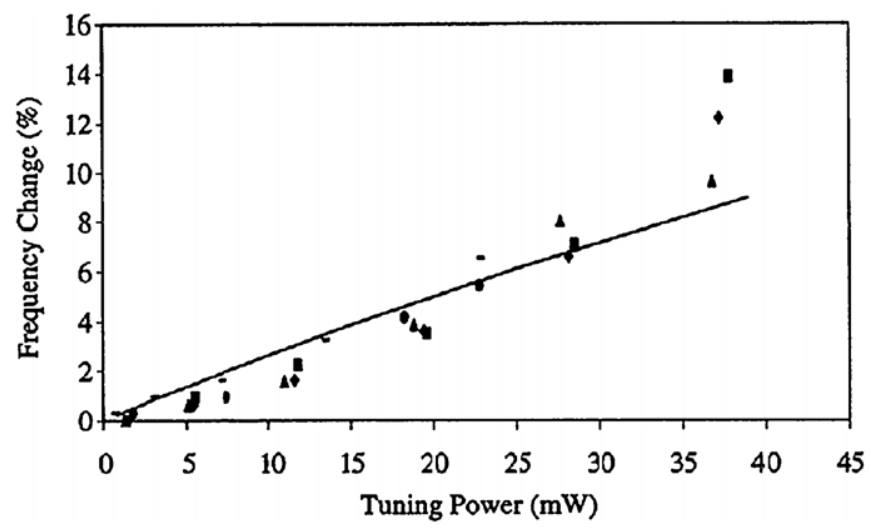

Figure 27. Measured frequency change versus tuning power [23].

The majority of cantilever-based micro-generators operate in the fundamental flexural mode (mode 1); the resonant

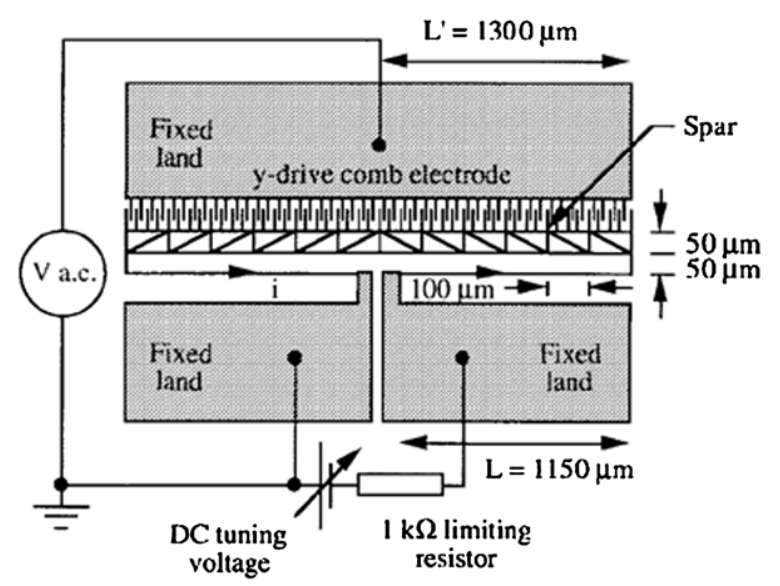

Figure 28. Layout and connection of laterally resonant comb-drive actuator used for tuning experiments [24].

frequency of a uniform cantilever in mode 1 with an axial load, $f_{r 1}{ }^{\prime}$, is given by

$$
f_{r 1}^{\prime}=f_{r 1} \cdot \sqrt{1+\frac{F}{F_{b}}} .
$$

The ratio of the tuned frequency to the original frequency is

$$
\frac{f_{r}^{\prime}}{f_{r}}=\sqrt{1+\frac{F}{F_{b}}} .
$$

The buckling load $F_{b}$ of a cantilever and a clampedclamped beam is given by equations (22) and (23), respectively [27]:

$$
\begin{aligned}
& F_{b}=\frac{\pi^{2} \cdot E \cdot w \cdot h^{3}}{48 \cdot l^{2}} \\
& F_{b}=\frac{\pi^{2} \cdot E \cdot w \cdot h^{3}}{3 \cdot l^{2}}
\end{aligned}
$$

where $E$ is Young's modulus of the material of the cantilever and $w, h$ and $l$ are the width, thickness and length of the cantilever, respectively.

Figure 32 shows the change in the resonant frequency of a cantilever with an axial load. It shows that a compressive 


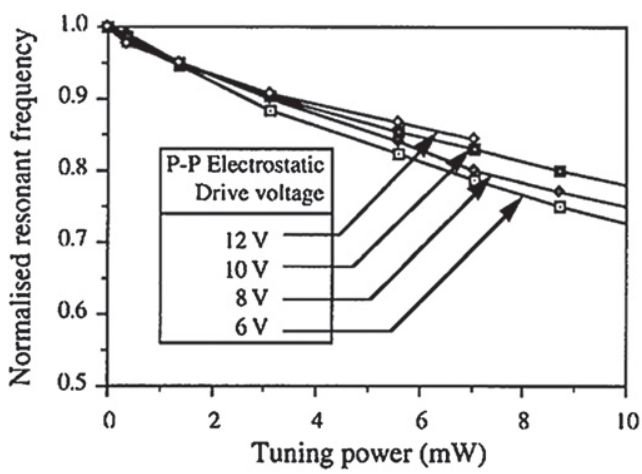

(a)

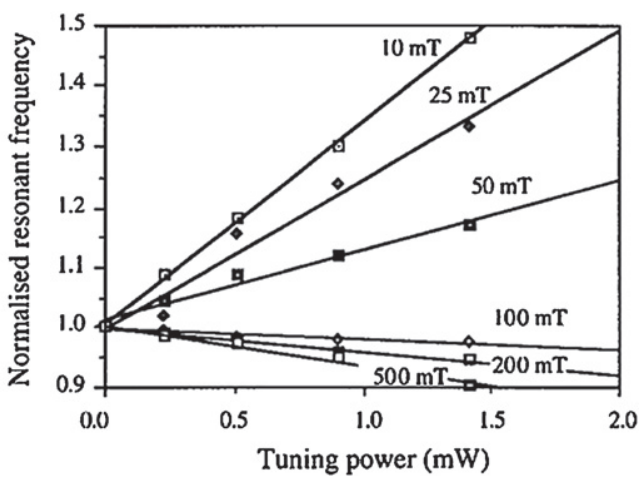

(b)

Figure 29. Variation of a resonant frequency with tuning power $(a)$ at a different electrostatic drive voltage and $(b)$ gas pressure [24].

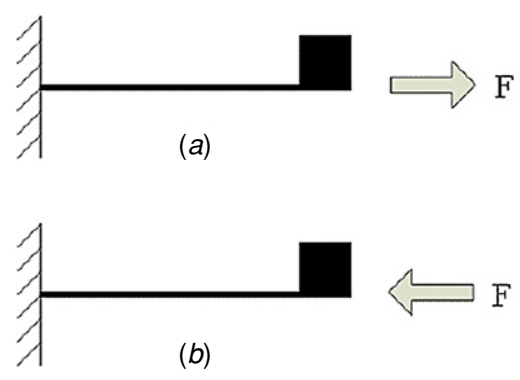

Figure 30. Axial tensile $(a)$ and compressive $(b)$ load on a clamped-free cantilever.

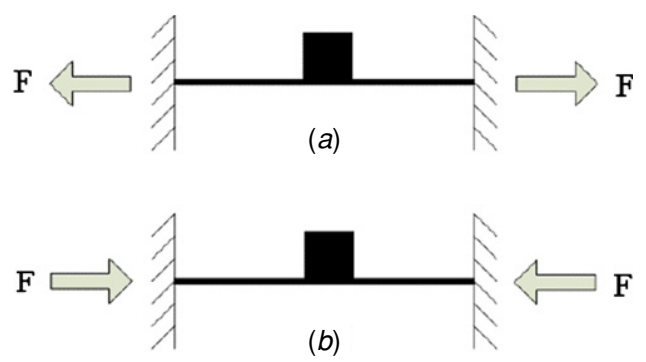

Figure 31. Axial tensile ( $a$ ) and compressive (b) load on a clamped-clamped beam.

load is more efficient in frequency tuning than a tensile load. If the compressive force is larger than the buckling load, the cantilever beam will buckle and no longer oscillate in mode 1. If a very large tensile force is axially applied to the cantilever, i.e. much greater than the buckling load, the resonant frequency will approach that of a straight tensioned cable as the force associated with the tension in the cantilever becomes much greater than the beam stiffness.

The following sections give examples of a clampedclamped beam and cantilever beam structures.

3.4.1. Clamped-clamped beam structures. Cabuz et al [28] realized resonant frequency tuning by applying an axial force on a micromachined resonant beam electrostatically as shown in figure 33. One end of the resonator was clamped on a fixed support while the other end was connected to a movable support. The moveable support could rotate around a torsion bar as a voltage was applied across two tuning electrodes.

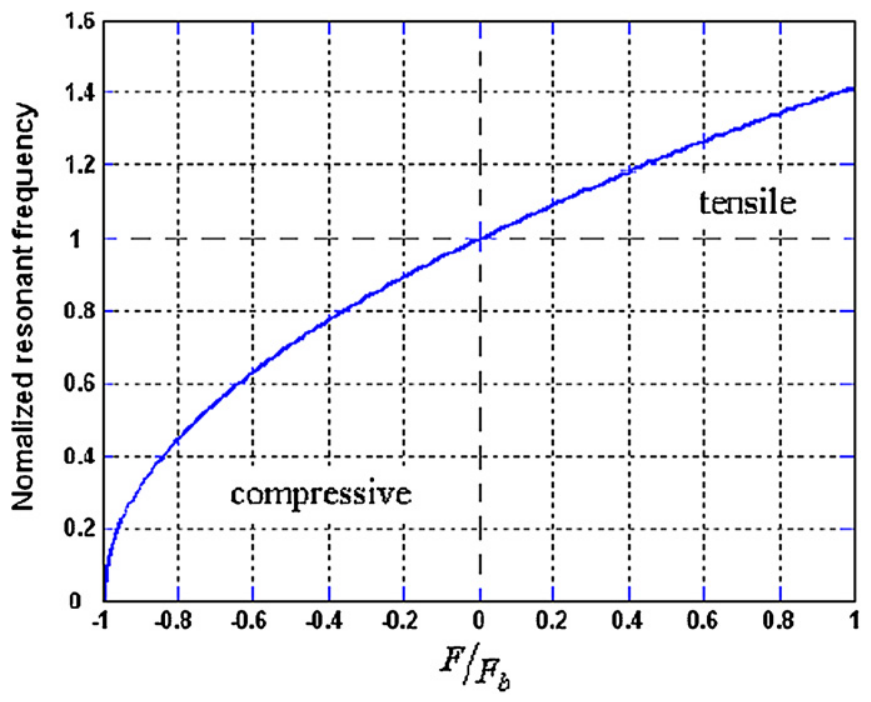

Figure 32. Normalized resonant frequency with variation of axial loads.

The torsion bar converted the vertical tuning motion into an axial force along the resonator. Upward rotation induces a compressive stress in the resonator while downward rotation induces a tensile stress. The tuning range was $16 \mathrm{~Hz}$ based on a centre frequency of $518 \mathrm{~Hz}$ (figure 34) with driving voltage from 0 to $16 \mathrm{~V}$. The dimensions of the resonator are $1000 \mu \mathrm{m}$ $\times 200 \mu \mathrm{m} \times 3 \mu \mathrm{m}$ and the dimensions of the movable support are $12.5 \mathrm{~mm}^{2} \times 0.3 \mathrm{~mm}$. This is an example of continuous tuning.

Leland and Wright [29] successfully tuned the resonant frequency of a vibration-based piezoelectric generator by manually applying an axial compressive preload directly on the cantilever using a micrometer (figures 35 and 36). The tuning range was from 200 to $250 \mathrm{~Hz}$. This device generated $300-400 \mu \mathrm{W}$ of power at an acceleration of $9.8 \mathrm{~m} \mathrm{~s}^{-2}$. It was determined that a compressive axial preload could reduce the resonance frequency of a vibration energy scavenger by up to $24 \%$, but it also increased the total damping (figure 37 ). The piezoelectric bimorph has dimensions of $31.7 \times \mathrm{mm} \times$ $12.7 \mathrm{~mm} \times 0.509 \mathrm{~mm}$ and the weight of the proof mass is $7.1 \mathrm{~g}$. This is an example of intermittent tuning, but it is not automated and has to be done manually. 


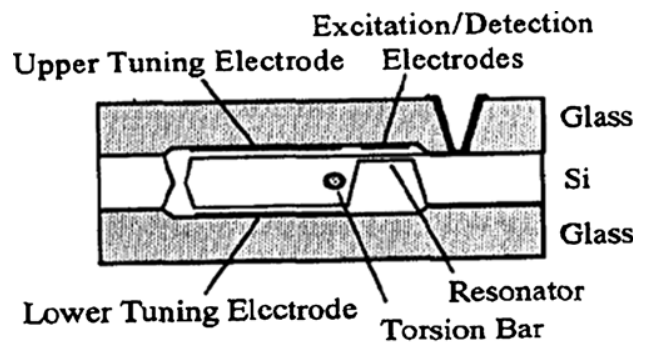

(a) Structure

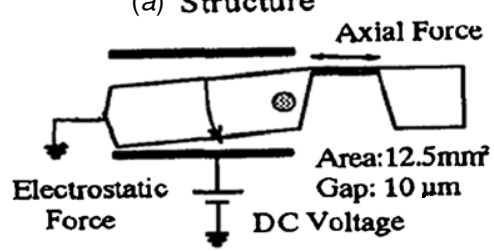

(b) Working Principle

Figure 33. Structure for fine resonance frequency tuning at a device level by an electrostatically induced axial force [28].

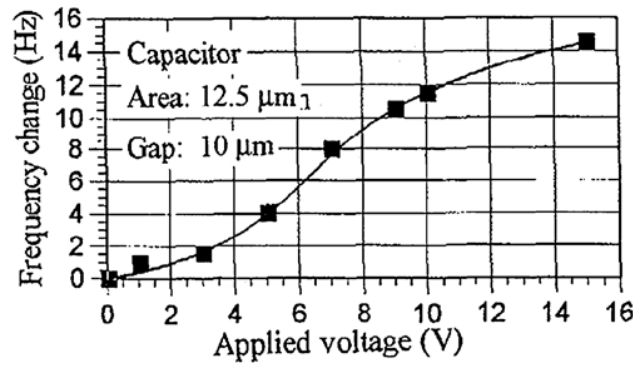

Figure 34. Resonant frequency change versus applied voltage [28].

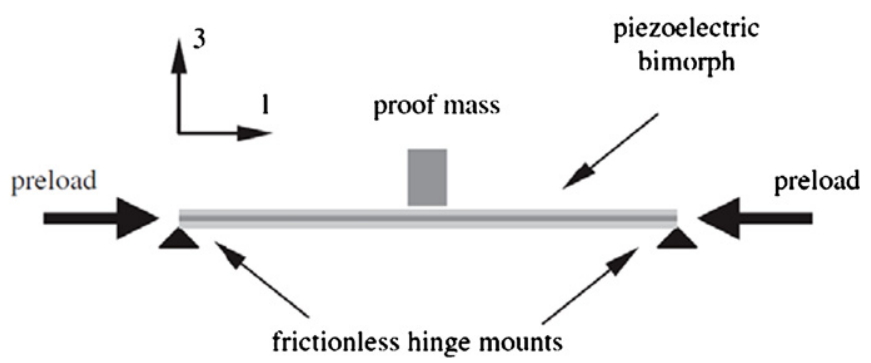

Figure 35. Schematic of a simply supported piezoelectric bimorph vibration energy scavenger [29].

3.4.2. Cantilever structures. Mukherjee [30] patented the idea of applying an axial force to a vibrating cantilever beamsensing element using the electrostatic force. The resonator consisted of two sets of comb-like structures (figure 38). The set closer to the anchor was used for sensing while the other set was used for frequency tuning. A voltage was applied between the two fixed tuning electrodes and the structure at the free end to apply an axial tensile or compressive end load to the cantilever. The resonant frequency of the beam was approximately $15.5 \mathrm{kHz}$. This is an example of continuous tuning which achieved a tuning range of $-0.6 \%$ to $3.3 \%$ of its untuned resonant frequency, i.e. about $600 \mathrm{~Hz}$. The cantilever buckled when $50 \mathrm{~V}_{\mathrm{dc}}$ was applied to provide a compressive force. This is an example of continuous tuning.

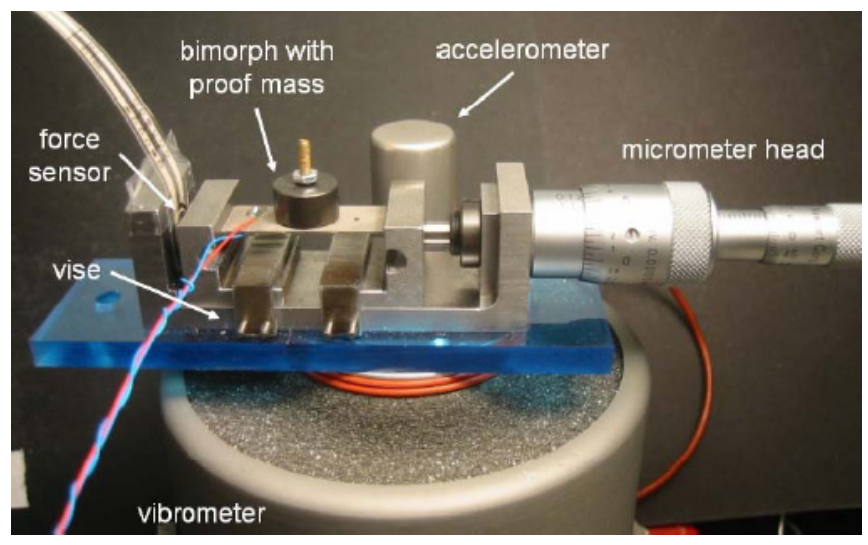

Figure 36. Experimental apparatus [29].

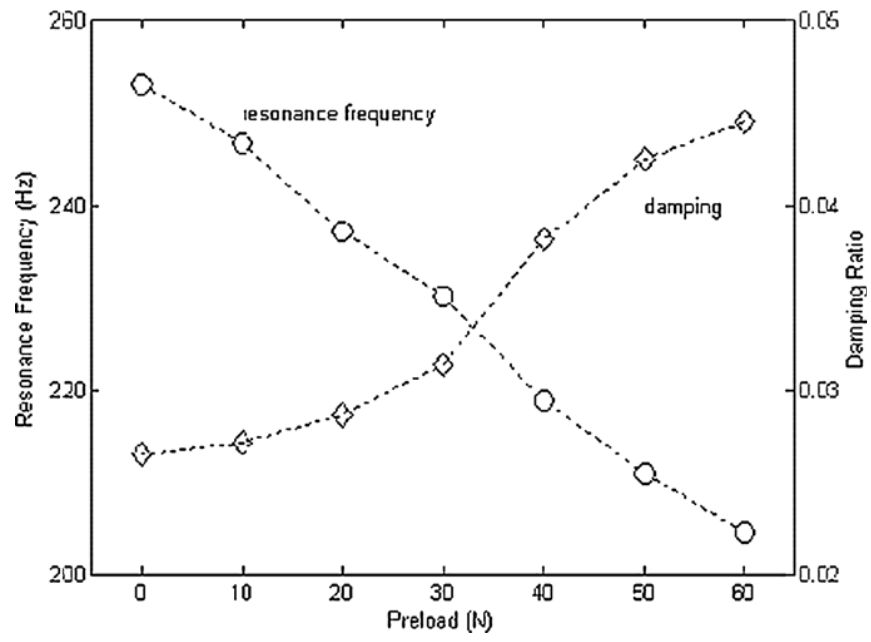

Figure 37. Resonance frequency and damping versus preload [29].

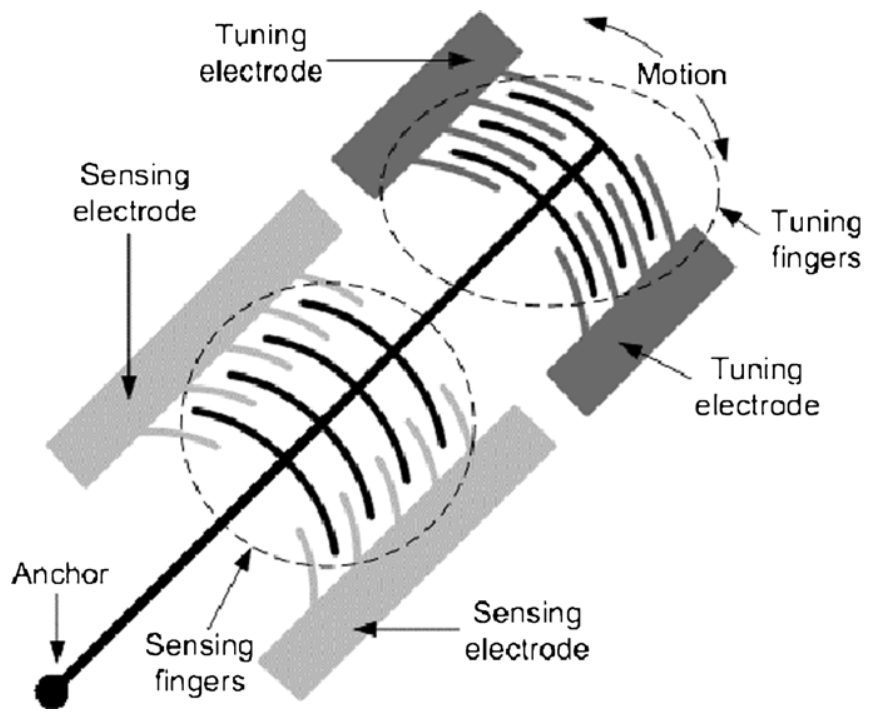

Figure 38. Resonator with actuator at the free end.

Hu et al [31] theoretically investigated an axial preloading technique to adjust the behaviour of a piezoelectric bimorph. Computational results show that resonance occurred when the natural frequency of the bimorph was adjusted to be 


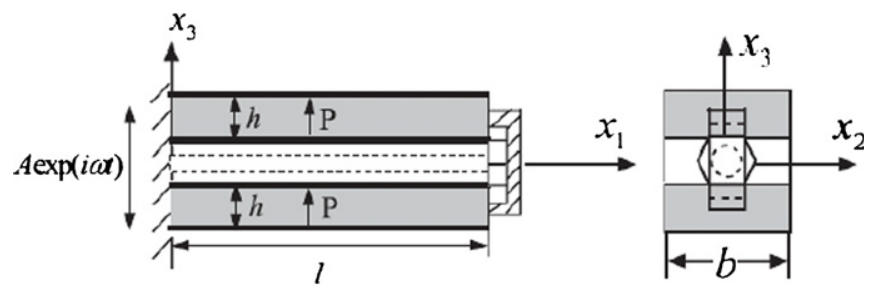

Figure 39. A method to apply axial preload to a piezoelectric bimorph [31].

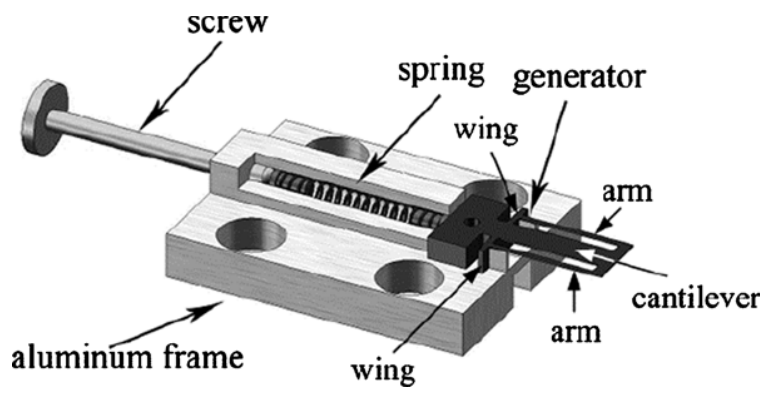

Figure 40. Schematic diagram of the test device [32].

adjacent to the external driving frequency by preloading. The mechanism for an axial preload to improve the bimorph performance at varying-frequency vibrations was examined in detail. A method for applying an axial preload to a piezoelectric bimorph was suggested and is shown in figure 39 . It comprises a mechanical bolt running through the central metal layer and fixed at the left-hand side edge wall. A capped stiff metal plate was attached to the bolt at the free end of the cantilever. A clockwise torsion of the bolt can produce a compressive preload to the bimorph, and conversely, an anticlockwise torsion of the bolt produces a force to pull the capping plate to move towards the right-hand side, which can generate a tensile preload to the bimorph. This is an example of manual intermittent tuning.

This principle was demonstrated by Eichhorn et al [32]. Figure 40 shows a schematic diagram of the test device. The piezoelectric generator consisted of a piezo-polymercomposite cantilever beam with arms on both sides to enable the application of an axial force to the free end of the beam. The arms were connected to the base with two wings. These wings were used to transmit the force to the arms, which in turn apply the load to the free end of the beam. The tuning force was applied by a screw and a steel spring. The axial load depends linearly on the deflection of the spring, which in turn was proportional to the number of revolutions of the screw. The spring pushes the whole generator base against two blocks of which the counter pressure generates the pre-stress in the arms and the stabilizing wings. The screw, spring and generator were all mounted on the same aluminium frame. This is another example of manual intermittent tuning.

In tests only a compressive load was applied. Figure 41 shows the test results of this generator under vibration level of $63.7 \mathrm{~m} \mathrm{~s}^{-2}$. It was found that with the increase of a compressive load, the resonant frequency, output voltage and the Q-factor reduced. By cutting notches on the wings the tuning efficiency

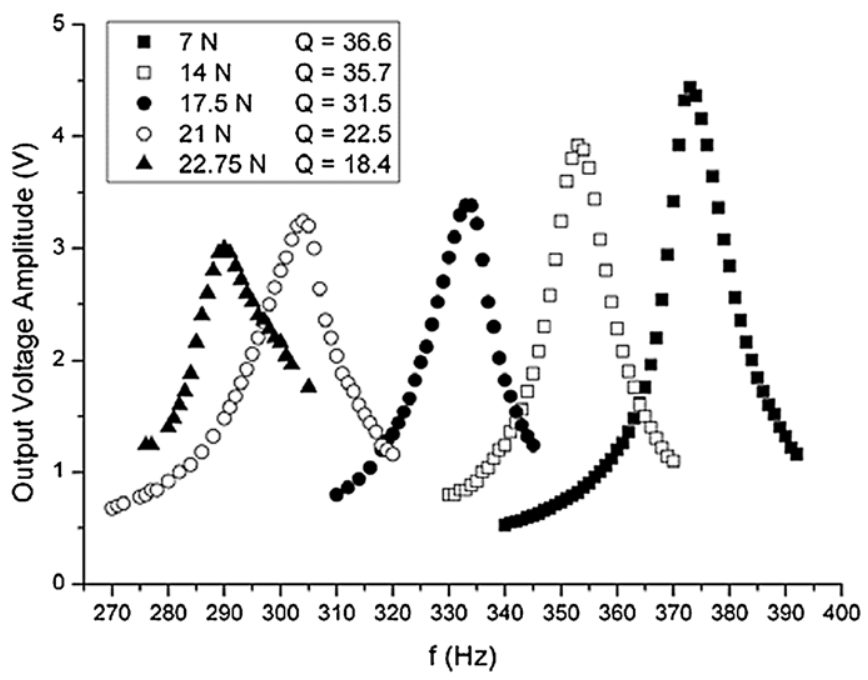

Figure 41. Test results under vibration of $63.7 \mathrm{~m} \mathrm{~s}^{-2}$ [32].

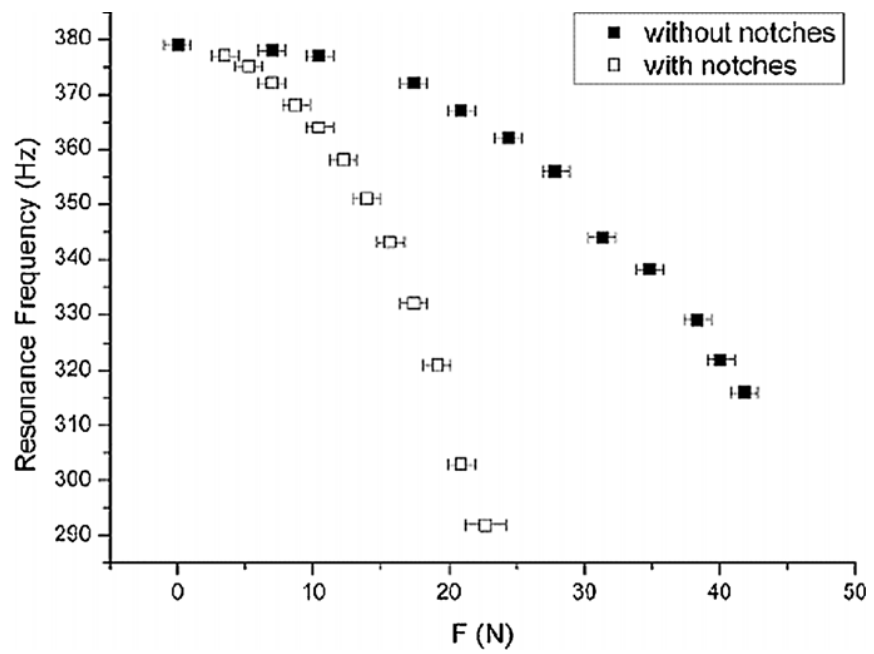

Figure 42. Comparison of tuning efficiency of wings with and without notches [32].

could be increased. With notches in the wings, a resonant frequency shift of more than $20 \%$ was achieved with a total force of $22.75 \mathrm{~N}$ (figure 42). The tuning range was from $290 \mathrm{~Hz}$ to $380 \mathrm{~Hz}$ with a compressive load up to $22.75 \mathrm{~N}$. The dimensions of the cantilever are $20 \mathrm{~mm} \times 5 \mathrm{~mm} \times 0.44 \mathrm{~mm}$ and the overall width of the device including arms is $13 \mathrm{~mm}$.

Another method of applying an axial load to a cantileverbased micro-generator is reported by Zhu et al [33] who presented a tunable electromagnetic vibration-based microgenerator with closed loop frequency tuning. Frequency tuning was realized by applying an axial tensile magnetic force to the micro-generator (figure 43).

The tuning force was provided by the attractive force between two tuning magnets with opposite poles facing each other. One magnet was fixed at the free end of a cantilever while the other was attached to an actuator and placed axially in line with the cantilever. The distance between the two tuning magnets was adjusted by the linear actuator. Thus, the axial load on the cantilever, and hence the resonant frequency, was changed. The areas where the two magnets face each 


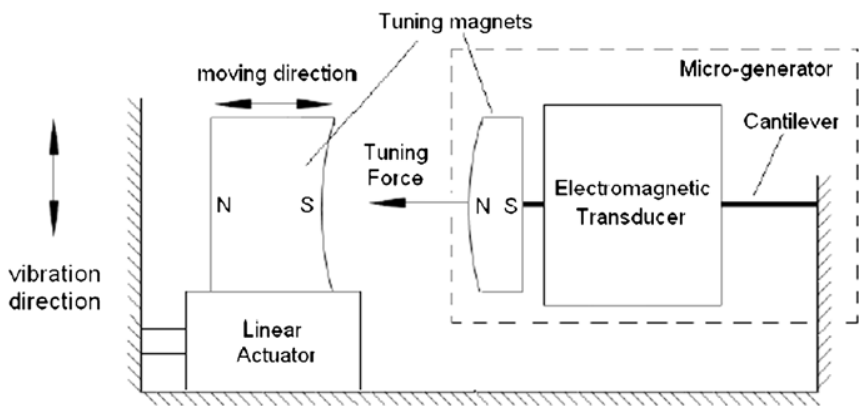

Figure 43. Schematic diagram of the tuning mechanism [33].

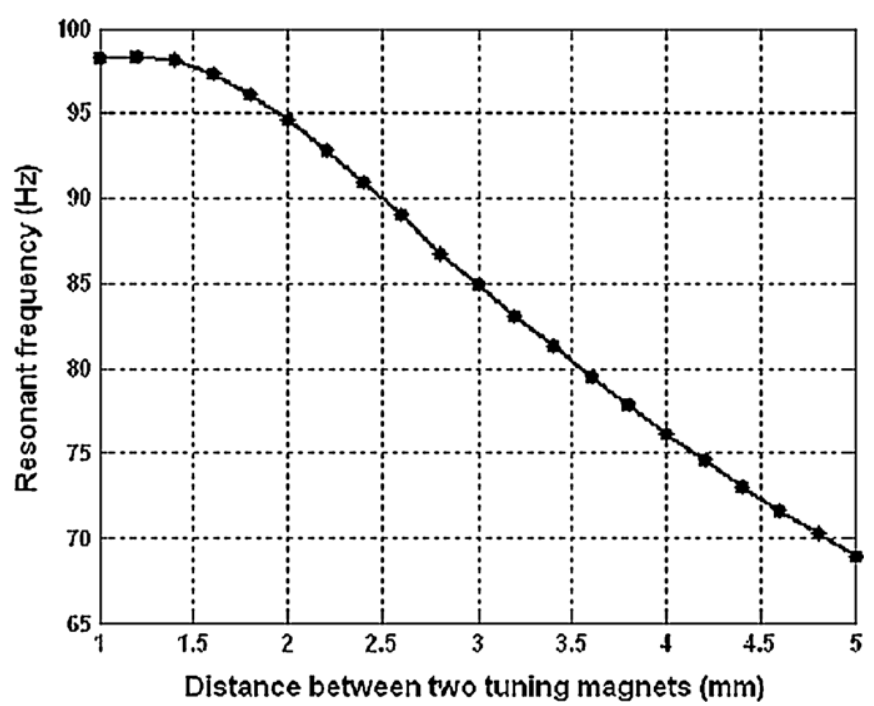

Figure 44. Resonant frequency with variance of distances between two tuning magnets [33].

other were curved to maintain a constant gap between them over the amplitude range of the generator. Figure 44 shows the test results of the resonant frequency variation of the distance between the two tuning magnets. The tuning range of the proposed micro-generator was from 67.6 to $98 \mathrm{~Hz}$ based on the original resonant frequency of $45 \mathrm{~Hz}$ by changing the distance between two tuning magnets from 5 to $1.2 \mathrm{~mm}$.

Experimentally, the generator produced a power of 61.6$156.6 \mu \mathrm{W}$ over the tuning range when it was excited at a constant vibration acceleration level of $0.588 \mathrm{~m} \mathrm{~s}^{-2}$. Furthermore, the tuning mechanism does not affect the damping of the micro-generator over most of the tuning range. However, when the tuning force became larger than the inertial force caused by vibration, total damping is increased and the output power is less than expected from theory (figure 45). This is the only example of automated intermittent tuning presented in the literature to date.

\section{Electrical tuning methods}

All the frequency tuning methods mentioned above are based on mechanical methods. The following sections describe methods to tune the resonant frequency of a vibration-based micro-generator electrically.

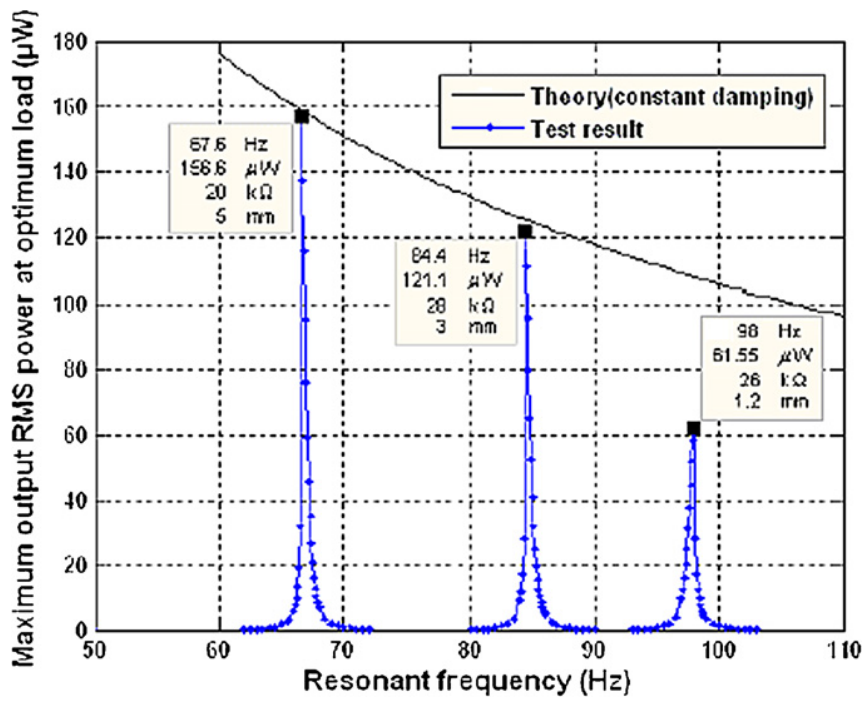

Figure 45. Power spectrum of the micro-generator (excited at $0.588 \mathrm{~m} \mathrm{~s}^{-2}$ ) [33].

Table 1. Coefficients of common piezoelectric materials [1, 34].

\begin{tabular}{lrrrr}
\hline & PZT-5 H & PZT-5 A & BaTiO $_{3}$ & PVDF \\
\hline$d_{31}\left(\times 10^{-12} \mathrm{C} \mathrm{N}^{-1}\right)$ & -274 & -171 & 78 & 23 \\
$\begin{array}{l}\text { Young's modulus }(\mathrm{GPa}) \\
\begin{array}{l}\text { Relative permittivity } \\
\left(\varepsilon / \varepsilon_{0}\right)\end{array}\end{array}$ & 50 & 50 & 67 & 2 \\
& & 1700 & 1700 & 12 \\
\hline
\end{tabular}

\subsection{Principle}

The basic principle of electrical tuning is to change the electrical damping by adjusting the load, which causes the power spectrum of the generator to shift. As all reported generators using electrical tuning are piezoelectric, in this section, only the piezoelectric micro-generator will be addressed. As resistive loads reduce the efficiency of power transfer and load inductances are difficult to be varied, it is most feasible to adjust capacitive loads to realize electrical tuning.

Figure 46 shows a schematic diagram of a bimorph piezoelectric generator with a mass, $m$, on the tip. $l_{b}$ and $l_{m}$ are the effective length of the cantilever and mass, respectively. $w$ is the width of the cantilever. $t_{p}$ and $t_{s}$ are the thickness of the piezoelectric layer and substrate layer, respectively, and $t_{g}$ is the distance from the centre of the substrate layer to the centre of the piezoelectric layer. Electrodes of the generator have been omitted in figure 47 . The resonant frequency of such a generator can be tuned by varying the capacitive load. Detailed theoretical analysis is shown in the appendix.

Figure 47 compares the resonant frequencies and power output of electrically tunable piezoelectric generators of different piezoelectric materials with variation of load capacitances. These generators are identical except for the piezoelectric material. The coefficients used in the simulation are listed in table 1.

The resonant frequency as well as the output power reduces with increasing load capacitance. It was found that PZT-5 $\mathrm{H}$ is the best of these four piezoelectric materials 


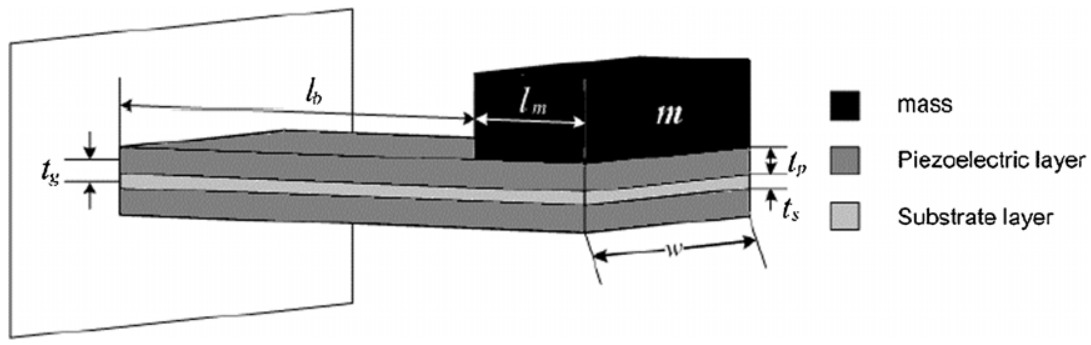

Figure 46. Piezoelectric bimorph generator.

for an electrically tunable piezoelectric generator. Important considerations relating to the tunability of the piezoelectric generator are as follows.

- The material of the substrate layer and mass does not affect the tunability.

- A piezoelectric material with higher Young's modulus, strain coefficient and smaller permittivity provides a larger tuning range.

- The ratio of the thickness of the piezoelectric layer to the thickness of the substrate layer should be small to increase the tuning range.

- The capacitance of the piezoelectric layer should be minimized to increase the tuning range.

- If both piezoelectric layers are used for tuning, connection of these two layers in parallel gives a larger tuning range than connection in series.

- The total damping should be kept low to increase the tuning range.

\subsection{Examples of electrically tunable micro-generators}

Wu et al [35] used this method to tune the resonant frequency of a generator composed of a piezoelectric bimorph cantilever. The upper piezoelectric layer was used for frequency tuning while the lower layer was used for energy harvesting. The tunable bandwidth of this generator was $3 \mathrm{~Hz}$ between $91.5 \mathrm{~Hz}$ and $94.5 \mathrm{~Hz}$. The charging time of the generator was compared with and without the tuning system. Experimentally, it was found that, when the device was excited under random frequencies from $80 \mathrm{~Hz}$ to $115 \mathrm{~Hz}$, the average harvesting output power of the generator with tuning was about $27.4 \%$ higher than that without tuning and the charging time was shortened by using the tuning system. These results showed a significant improvement of the average harvested power output by using an electrical tuning method.

Charnegie [36] presented another piezoelectric microgenerator based on a bimorph structure and adjusted its load capacitance. Again, one piezoelectric layer was designed for energy harvesting while the other is used for frequency tuning (figure 48).

The test results showed that if only one layer was used for frequency tuning (figure 49(a)), the resonant frequency can be tuned an average of $4 \mathrm{~Hz}$ with respect to the untuned frequency of $350 \mathrm{~Hz}$, i.e. $1.14 \%$ tuning by adjusting the load capacitance from 0 to $10 \mathrm{mF}$ (figure $50(a)$ ). If both layers were used for frequency tuning (figure $49(b)$ ), the tuning range was an average of $6.5 \mathrm{~Hz}$, i.e. $1.86 \%$ of tuning by adjusting the same amount of the load capacitance (figure 51(a)). It was found that if one layer was used for tuning and the other for energy harvesting (figure 49(a)), the output power did not reduce with the increase of the load capacitance (figure 50(b)). However, if both frequency tuning and energy harvesting were achieved using the same layer (figure 49(b)), the output power decreased when the load capacitance became larger (figure 51(b)).

\section{Strategies to widen bandwidth}

The other commonly used solution to increase the operational frequency range of a vibration energy-harvesting generator is to widen the bandwidth. To date, strategies to widen the bandwidth include using a generator array consisting of small generators with different resonant frequencies, introducing an amplitude limiter to the device, using coupled oscillators, employing nonlinear and bi-stable structures and designing a large generator with a large inertial mass and high degree of damping. In this section, details of generator array, amplitude limiter and nonlinear and bi-stable structures will be covered. The strategy of employing a single large generator will not be detailed as it can be simply described using equation (1) while it will be considered in the comparison of different strategies later in this review.

\subsection{Generator array}

In this method, the bandwidth is widened by designing a generator consisting of an array of small generators, each of which has different dimensions and mass and hence different resonant frequencies (figure 52). Thus, the assembled generator has a wide operational frequency range whilst the Qfactor does not decrease. Figure 53 shows the power spectrum of a generator array which is a combination of the power spectra of each small generator. The frequency band of the generator is thus essentially increased. The drawback of this approach is the added complexity of fabricating an array of generators and the increased total volume of the device depending upon the number of devices in the array.

Shahruz [37] developed a device which consisted of a set of cantilever beams with proof masses at their tips. The dimensions of each beam and mass were different and were chosen appropriately to make the whole device work as a band-pass filter. Each beam is an individual generator. Since these beams had different resonant frequencies, the 

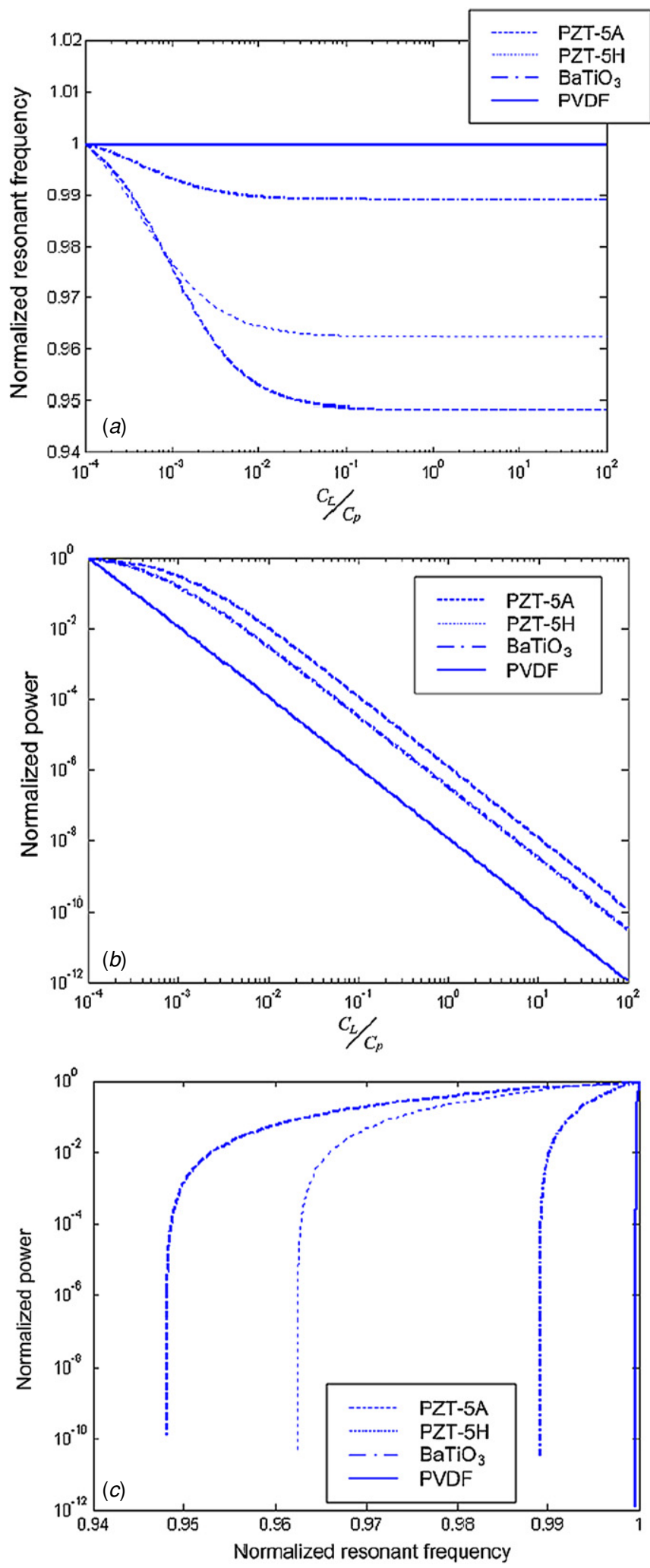

Figure 47. Performance of a piezoelectric generator with different piezoelectric materials: $(a)$ resonant frequency, $(b)$ output power, (c) output power versus resonant frequency.

filter could automatically 'select' one beam to resonate at one particular frequency based on the driving vibration frequency. A generator based on such a filter will work at

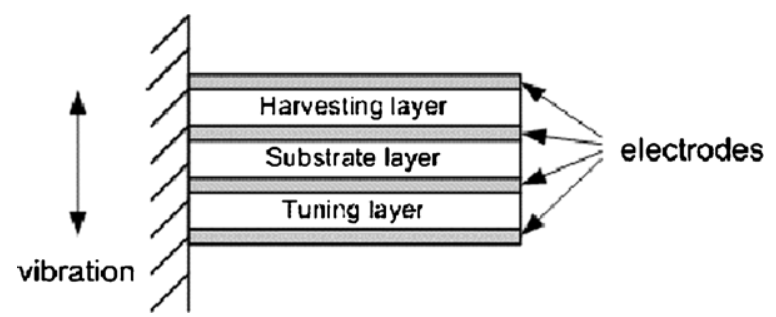

Figure 48. Piezoelectric bimorph used for electrical frequency tuning.

various frequencies, i.e. effectively widening the operational frequency range.

Xue et al [38] presented an approach for designing broadband piezoelectric harvesters by integrating multiple piezoelectric bimorphs with different aspect ratios into a system, primarily with different thicknesses of piezoelectric layers, $h$. Figure 54 shows a schematic diagram of the array.

The effect of connecting piezoelectric bimorphs in series and in parallel on improving energy-harvesting performance was discussed. It was found that the bandwidth of a generator can be widened by connecting multiple piezoelectric bimorphs with different aspect ratios in series. In addition, the bandwidth of the generator can be shifted to the dominant frequency domain of the ambient vibrations by increasing or decreasing the number of piezoelectric bimorphs in parallel. Numerical results showed that the bandwidth of the piezoelectric energyharvesting devices can be tailored by the connection patterns (i.e. in series and in parallel) among piezoelectric bimorphs (figures 55 and 56).

Feng et al [39] presented a micromachined piezoelectric generator with a wide vibration bandwidth. The device was designed to achieve an optimal figure of merit (FOM) which

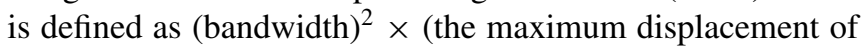
cantilever structures under a given acceleration under static conditions). The dimensions of the generator are $3 \mathrm{~mm} \times$ $3 \mathrm{~mm} \times 5 \mathrm{~mm}$ and it consisted of four cantilever structures connected in parallel, which were fabricated to achieve a flexible membrane with the minimum residual stress capable of a large displacement. Each cantilever had a different mass or centre of gravity and so a different resonant frequency (figure 57). The designed generator was targeted at producing microwatts to milliwatts in a wide mechanical vibration range from 300 to $800 \mathrm{~Hz}$ (figure 58) but no test results were reported to date.

A multifrequency piezoelectric generator intended for powering autonomous sensors from background vibrations was presented by Ferrari et al [40]. The generator consisted of multiple bimorph cantilevers with different natural frequencies of which the rectified outputs were fed to a single storage capacitor. A generator with three commercially available piezoelectric bimorph cantilevers was examined. Each cantilever has the same dimensions of $15 \mathrm{~mm} \times 1.5 \mathrm{~mm} \times$ $0.6 \mathrm{~mm}$ and different masses, $1.4 \mathrm{~g}, 0.7 \mathrm{~g}$ and $0.6 \mathrm{~g}$, respectively. The generator was used to power a batteryless sensor module that intermittently read the signal from a passive sensor and sent the measurement information via RF 


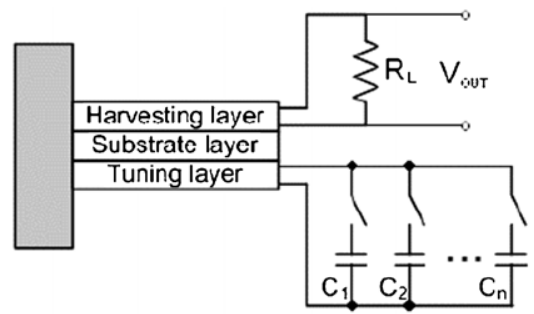

Capacitor array

(a)

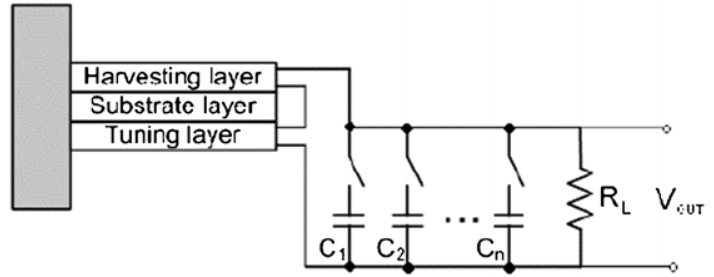

Capacitor array

b)

Figure 49. Frequency tuning and energy harvesting using $(a)$ the same layer and $(b)$ different layers.

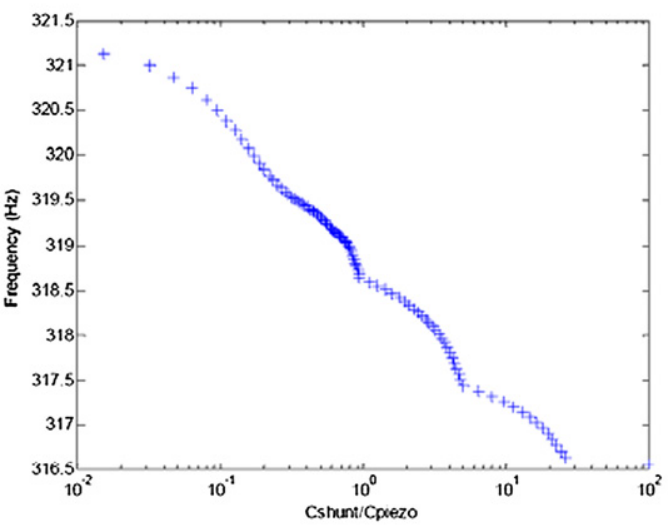

(a)

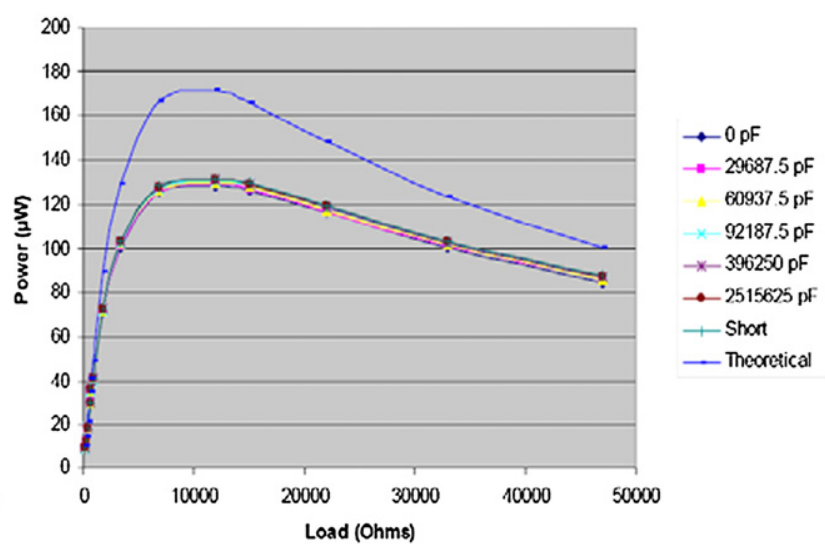

(b)

Figure 50. Resonant frequency $(a)$ and output power $(b)$ versus load capacitance while tuning and energy harvesting in different layers [36].

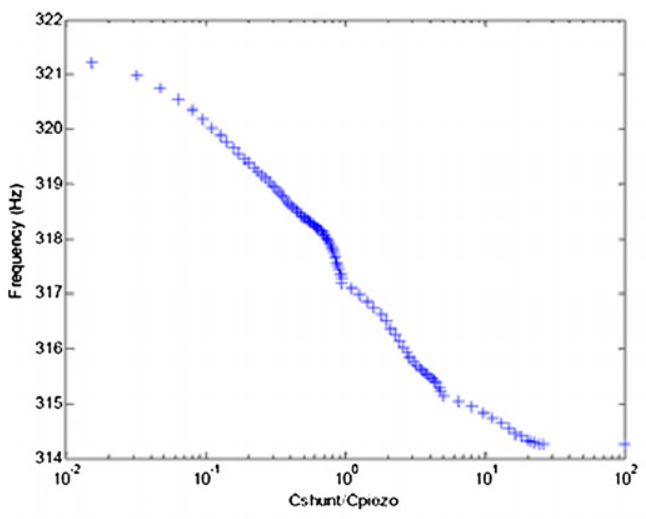

(a)

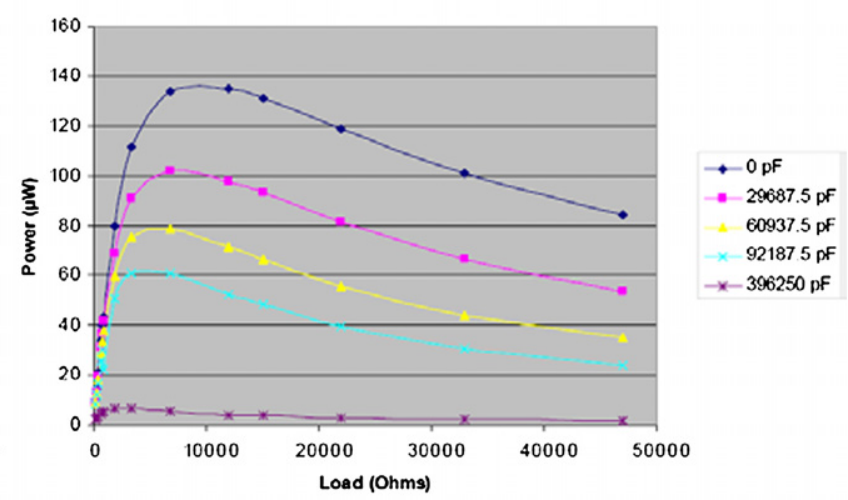

(b)

Figure 51. Resonant frequency $(a)$ and output power $(b)$ versus load capacitance while tuning and energy harvesting in the same layers [36].

transmission, forming an autonomous sensor system. It was found that the sensor module could be sufficiently powered, thereby triggering the transmission, even for an excitation frequency that was off resonance for all of the cantilevers. At resonance none of the cantilevers used alone was able to provide enough energy to operate the sensor module. Experimentally, it showed that a generator array operating with wideband frequency vibrations provides improved overall energy conversion over a single generator at the expense of larger volume.

Sari et al [41] reported a micromachined electromagnetic generator with a wide bandwidth. The generator consists of a series of cantilevers with various lengths and hence resonant frequencies (figure 59). These cantilevers are distributed in a $12.5 \mathrm{~mm} \times 14 \mathrm{~mm}$ area. The length of the cantilevers increased gradually so that the cantilevers have overlapping frequency spectra with the peak powers at similar but different frequencies. This resulted in a widened bandwidth as well as an increase in the overall output power.

The generator used an electromagnetic transducer to transduce power. A large magnet was fixed in the middle of the cantilever array and coils were fabricated by sputtering and patterning metal on top of the cantilevers. The coils moved with ambient vibration relatively to the static magnet 


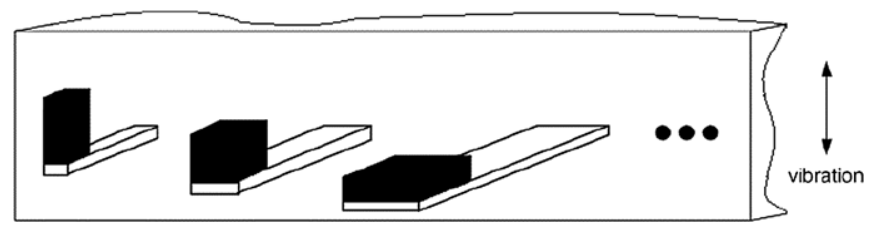

Figure 52. A mechanical band-pass filter with a set of cantilever beams.

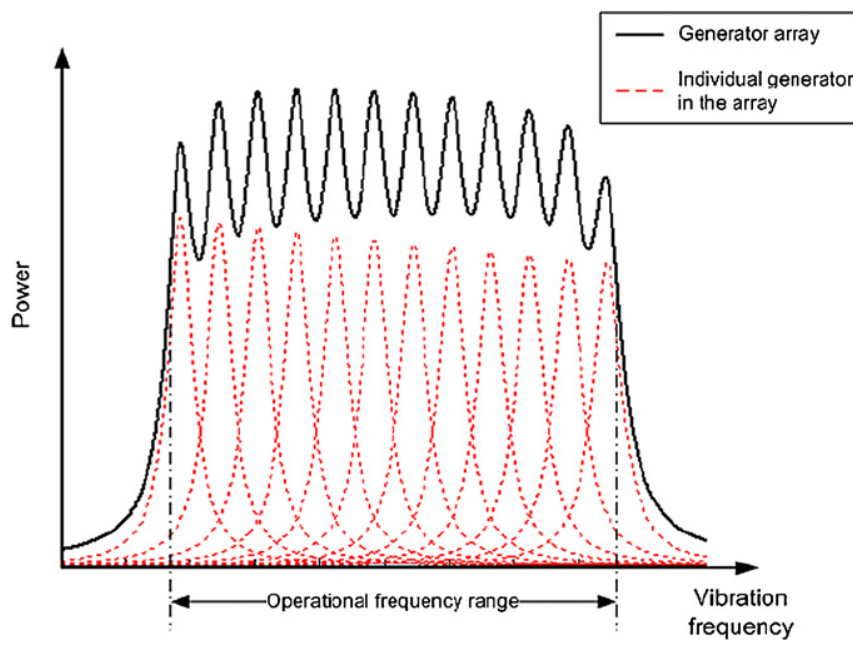

Figure 53. Power spectrum of a generator array.

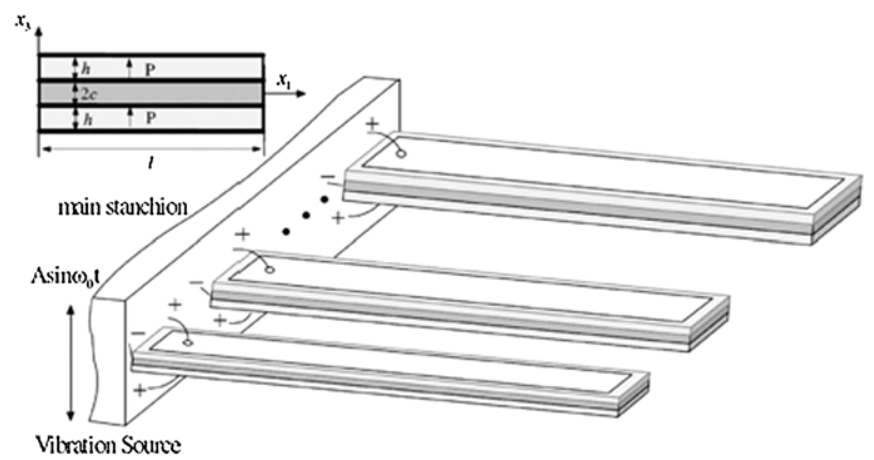

Figure 54. Schematic illustration of a piezoelectric bimorphs harvesting system [38].

and they were connected in series. Experimentally, the device generated $0.5 \mu \mathrm{W}$ continuous power at $20 \mathrm{mV}$ voltage between 3.3 and $3.6 \mathrm{kHz}$ of ambient vibration. Figure 60 shows the power spectrum of this generator.

\subsection{Amplitude limiter}

Another method of increasing the bandwidth of a vibrationbased micro-generator was reported by Soliman et al [42]. The bandwidth of the device was increased by using a mechanical stopper (amplitude limiter) to limit the amplitude of the resonator (figures 61 and 62). The theory behind this method is complex and details can be found in [42]. It was found that this method increases the bandwidth of the generator during an up-sweep, i.e. when the excitation frequency was gradually increased. The bandwidth remained the same in a down-sweep, i.e. when the excitation frequency was gradually

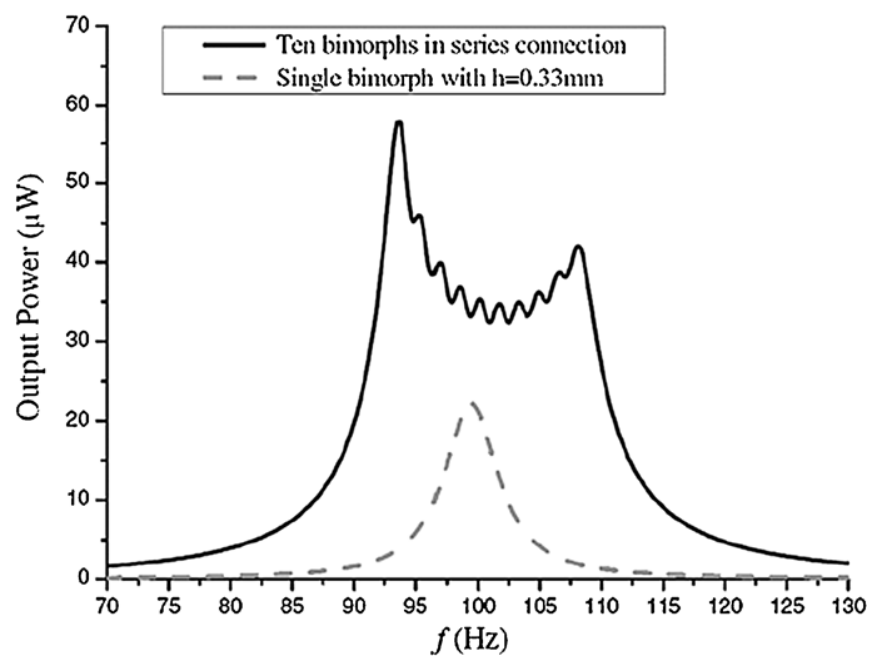

Figure 55. Comparison of a power spectrum for a single piezoelectric bimorph and ten piezoelectric bimorphs in series with various thicknesses of piezoelectric layer, $h$ [38].

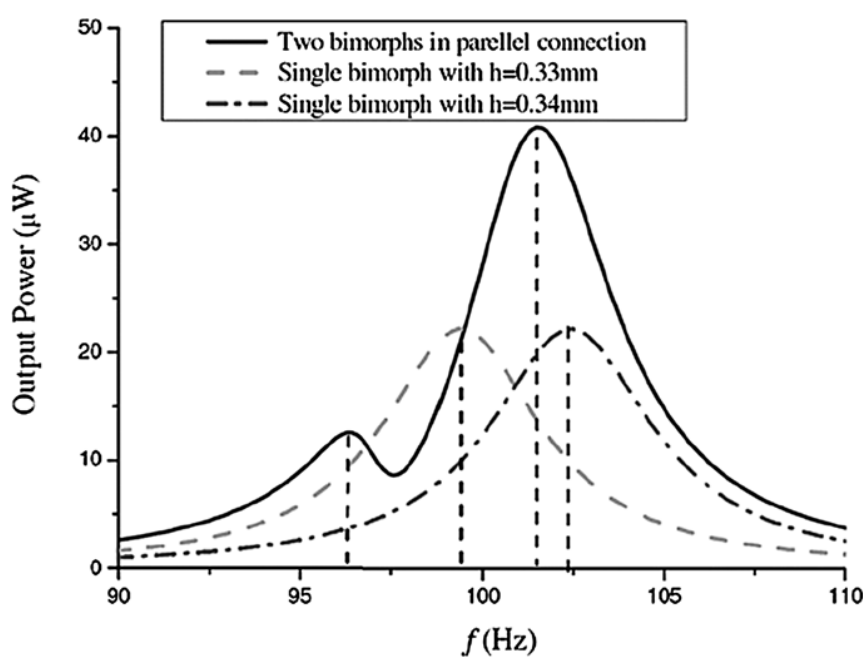

Figure 56. Effect of piezoelectric bimorphs in parallel on harvester performance [38].

reduced. Experimental measurements showed that the upsweep bandwidth was $240 \%$ wider than that of the architecture without a stopper at the half-power level, but the maximum output voltage was $30 \%$ less (figure 63 ). The dimensions of the cantilever are $45.3 \mathrm{~mm} \times 10 \mathrm{~mm} \times 1.02 \mathrm{~mm}$ and the mass is extrapolated to be $2.92 \mathrm{~g}$.

\subsection{Coupled oscillators}

The method of widening the operational bandwidth of the MEMS generator was reported by Petropoulos et al [43]. The proposed generator employs a pair of coupled oscillators that consist of two springs, two masses and two dampers. The first spring connects the inertial frame and the first mass while the second spring connects the two masses while each mass has a damper to the frame as shown in figure 64. The analytic model shows that this type of generators has flat response for power generation over a wider frequency range. However, 

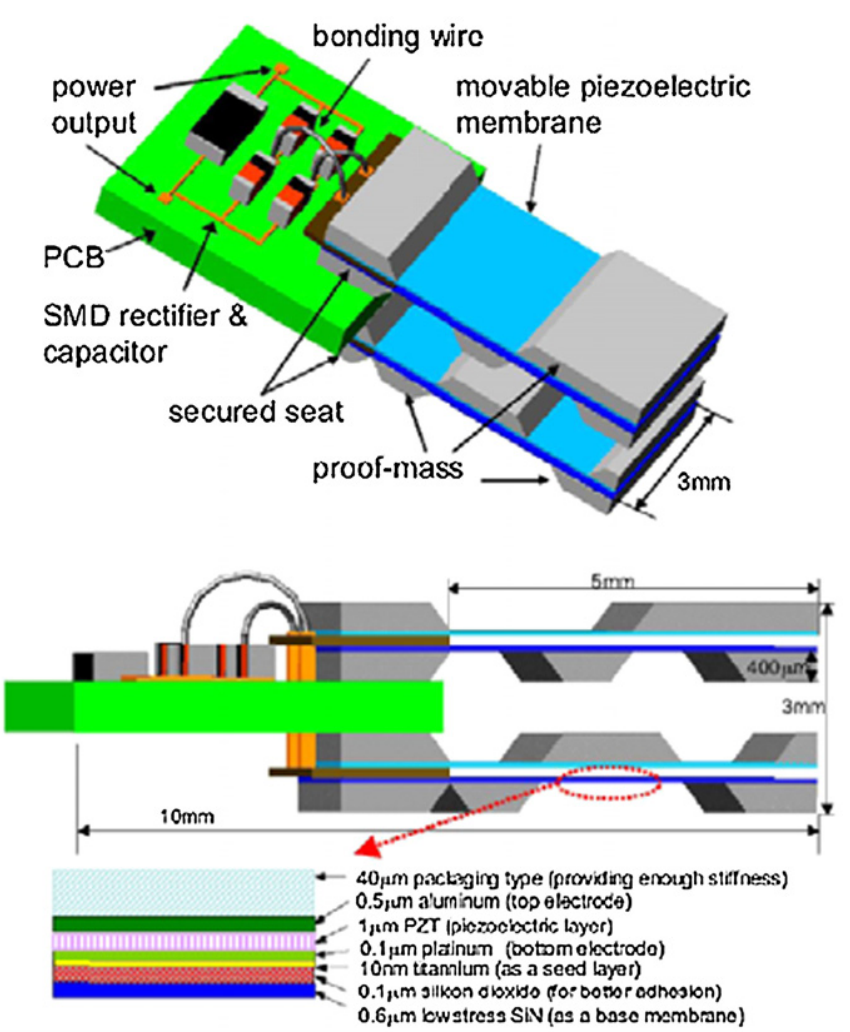

Figure 57. Conceptual diagram of the piezoelectric wide-bandwidth micro-generator [39].

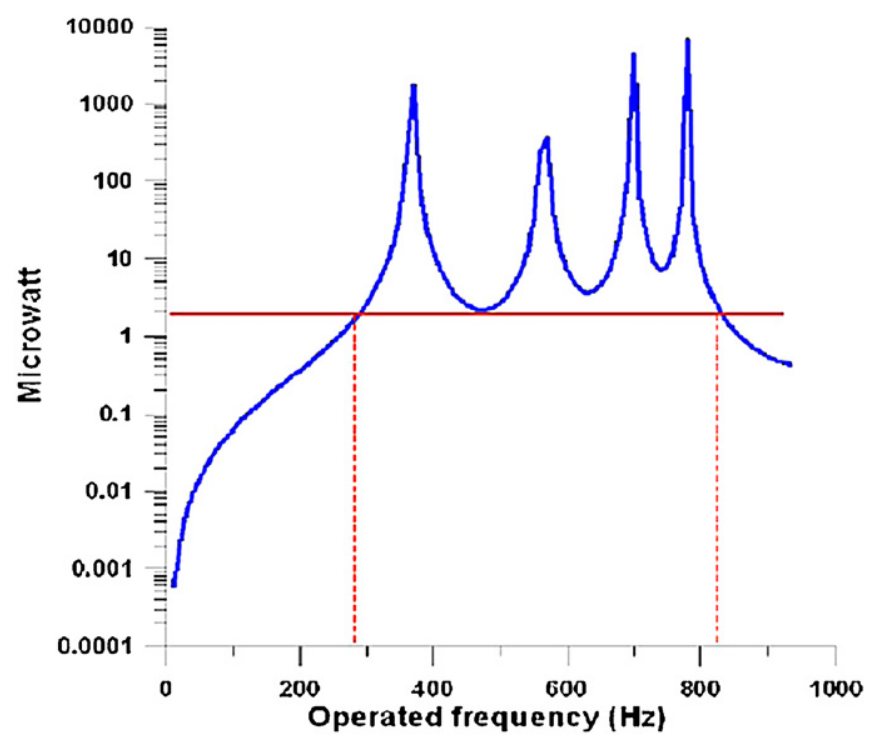

Figure 58. Estimated power generation with the power range of $\mu \mathrm{W}$ to $\mathrm{mW}$ in a wide bandwidth [39].

the maximum output power of the generator is significantly decreased than the generator with one mass (figure 65).

\subsection{Nonlinear generators}

The theory of vibration energy harvesting using nonlinear generators was investigated by Ramlan et al [44]. Instead

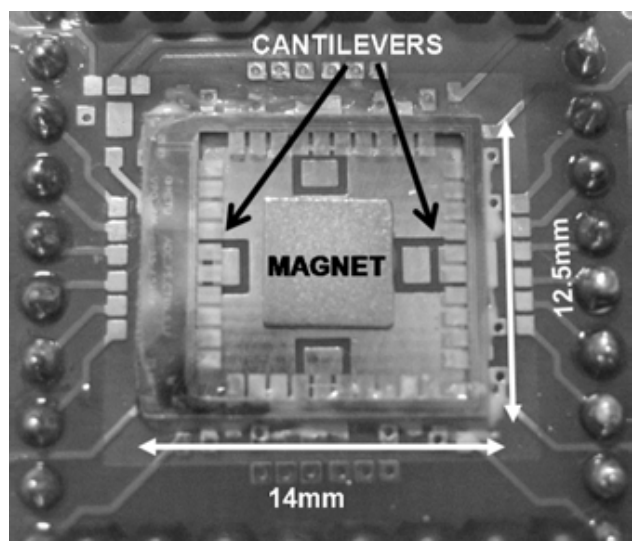

Figure 59. Photograph of a wide-band electromagnetic generator [41].

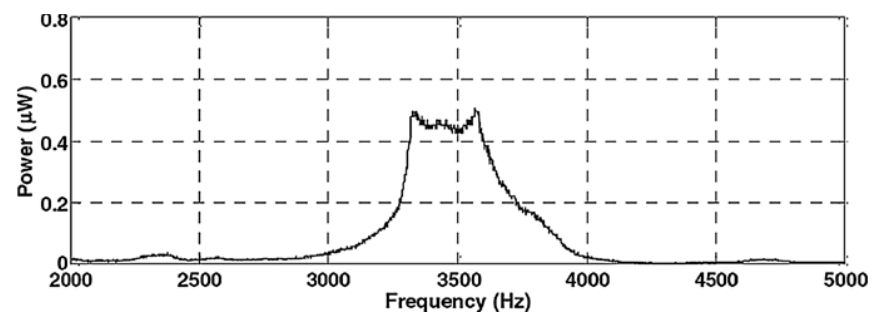

Figure 60. Power spectrum of Sari's generator [41].

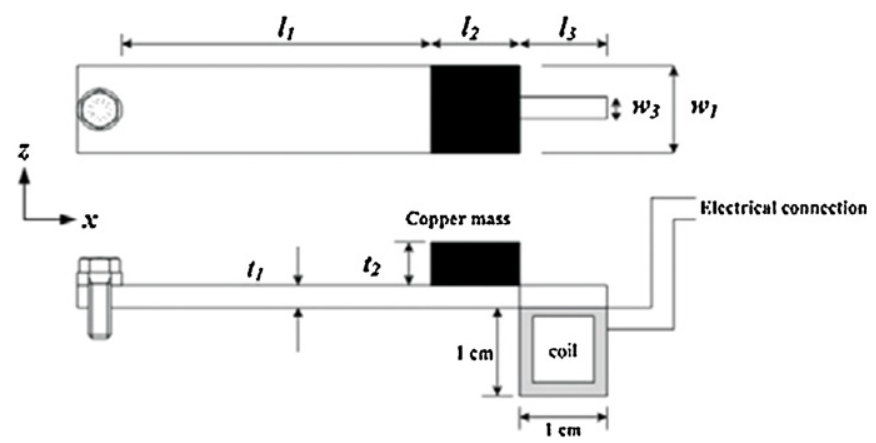

Figure 61. Top and side views of the device [42].

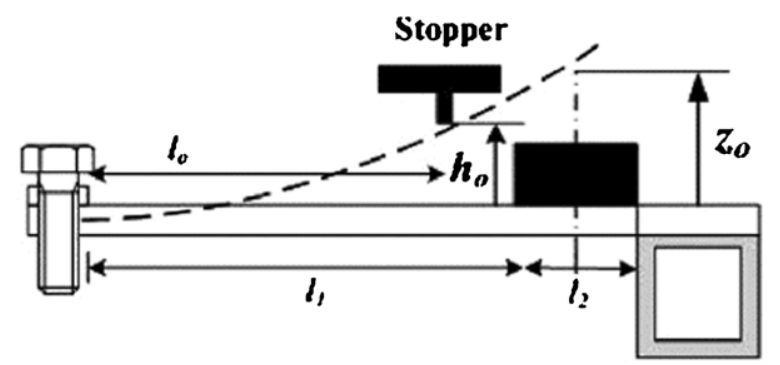

Figure 62. Increase the bandwidth using a stopper [42].

of using a conventional second-order model as (1), nonlinear generators were modelled using Duffing's equation as follows:

$$
m \frac{\mathrm{d}^{2} z(t)}{\mathrm{d} t^{2}}+b \frac{\mathrm{d} z(t)}{\mathrm{d} t}+k z(t)+k_{n}[z(t)]^{3}=-m \frac{\mathrm{d}^{2} y(t)}{\mathrm{d} t^{2}}
$$




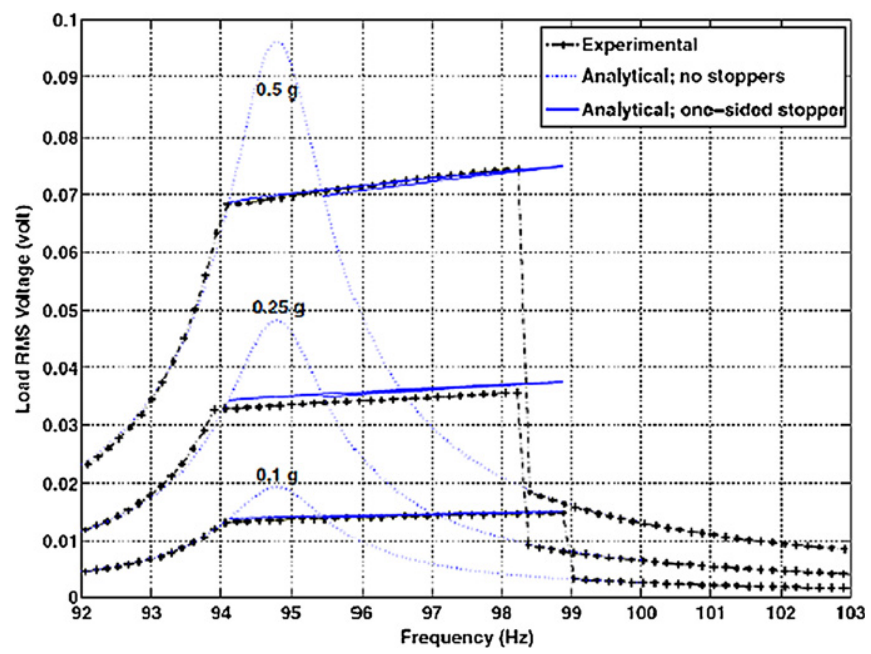

Figure 63. Voltage on load versus excitation frequency [42].

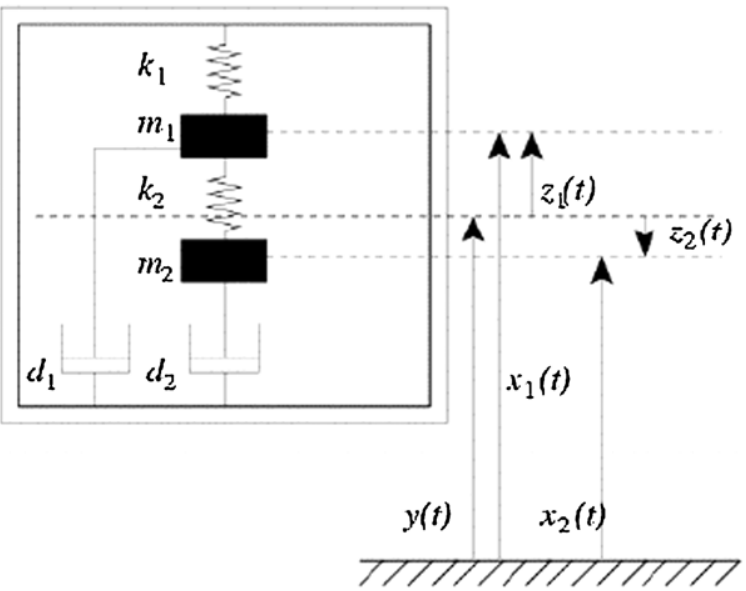

Figure 64. Schematic model of a coupled oscillator [43].

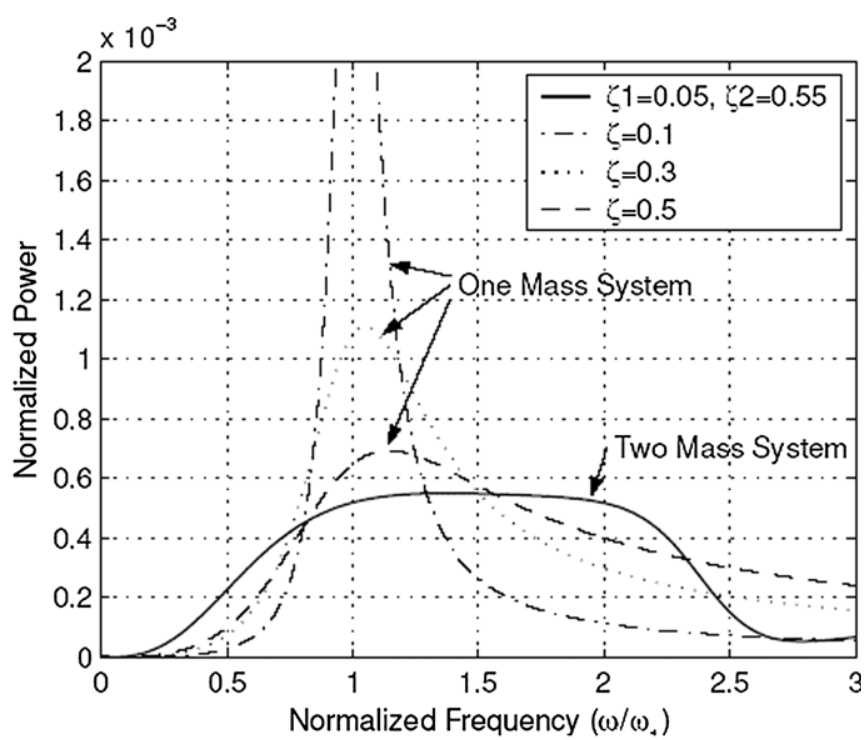

Figure 65. Power spectrum for an optimized two-mass system and for an equivalent one-mass system with various $\zeta$ values [43].
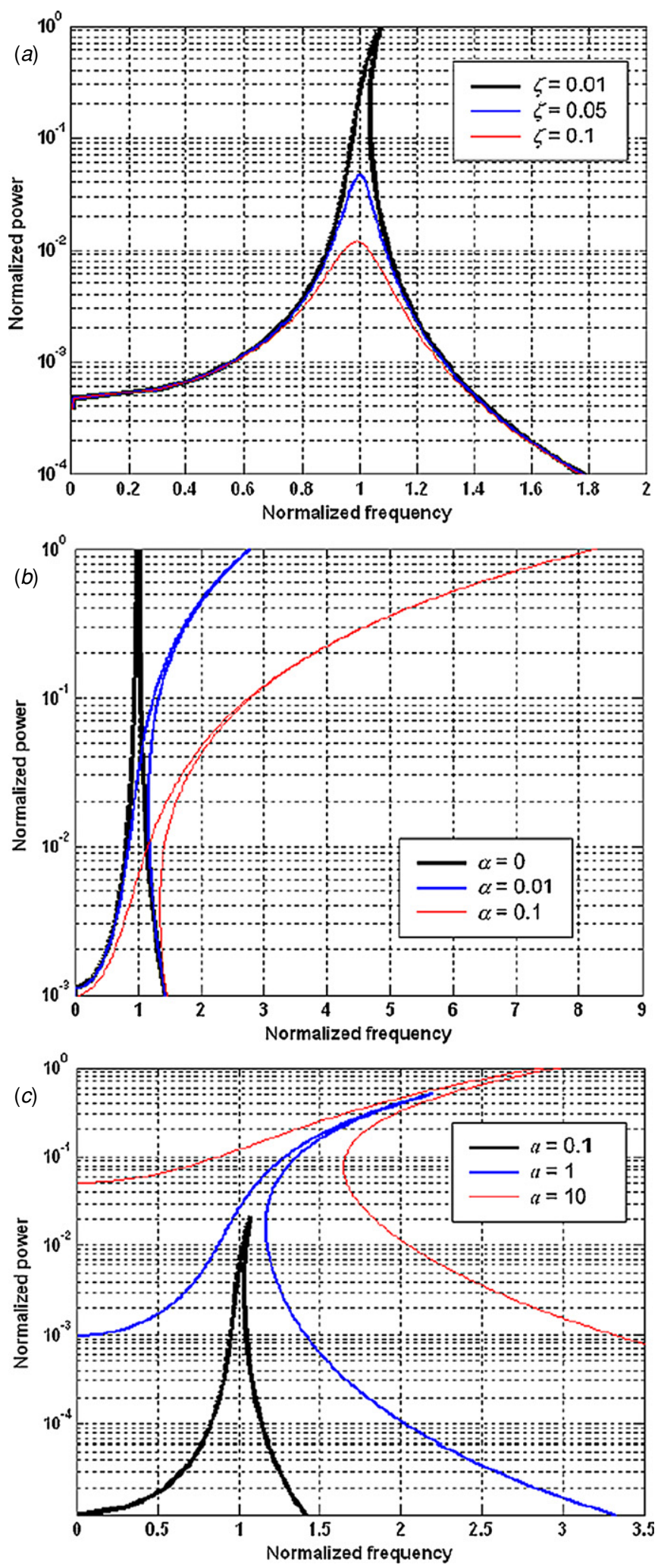

Figure 66. Power spectrum of nonlinear generators: $(a)$ various damping ratios, $(b)$ various nonlinearities, $(c)$ various input accelerations.

where the spring force is the combination of the linear force, $k z(t)$, and the nonlinear force, $k_{n}[z(t)]^{3}$. Solving (24) gives the frequency-amplitude relationship as 


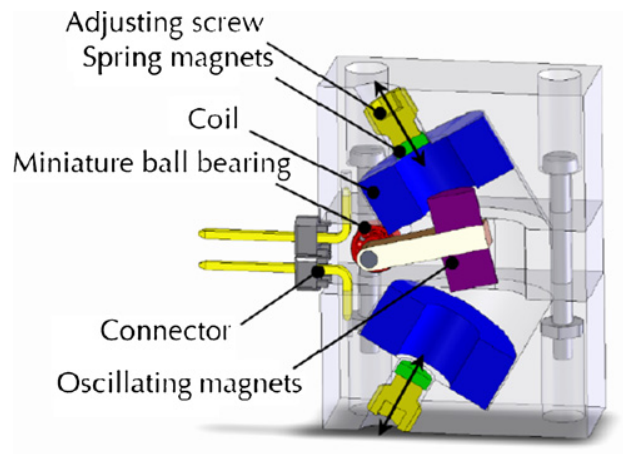

Figure 67. Half-section of the device [45].

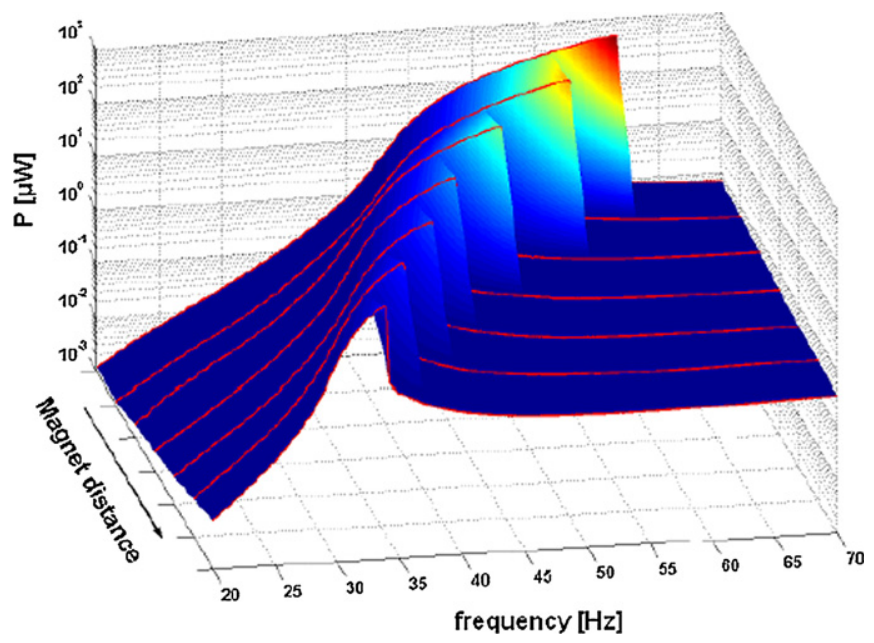

Figure 68. Measured output power [45].

$\Omega_{a}=\left(\frac{3}{4} \alpha Z^{2}+\left(1-2 \zeta^{2}\right)-\frac{\left(a^{2}-3 \alpha \zeta^{2} Z^{4}+4 \zeta^{2} Z^{2}\left(\zeta^{2}-1\right)\right)^{\frac{1}{2}}}{Z}\right)^{\frac{1}{2}}$

$\Omega_{b}=\left(\frac{3}{4} \alpha Z^{2}+\left(1-2 \zeta^{2}\right)+\frac{\left(a^{2}-3 \alpha \zeta^{2} Z^{4}+4 \zeta^{2} Z^{2}\left(\zeta^{2}-1\right)\right)^{\frac{1}{2}}}{Z}\right)^{\frac{1}{2}}$

where $\alpha$ is proportional to the nonlinear spring factor, $k_{n}, Z$ is the amplitude of the proof mass, $\zeta$ is the damping factor and $a$ is the normalized excitation acceleration.

Such devices have a hardening spring which has the effect of shifting the resonant frequency. Numerical and analytical studies showed that a device with a hardening spring has a larger bandwidth over which power can be harvested due to the shift in the resonance frequency. Their analysis also showed that the bandwidth of the hardening system depends on the damping ratio, the nonlinearity and the input acceleration (figure 66). Ideally, the maximum amount of power harvested by a system with a hardening stiffness is the same as the maximum power harvested by a linear system, irrespective of the nonlinearity, although this possibly occurs at a different frequency depending on the nonlinearity. It

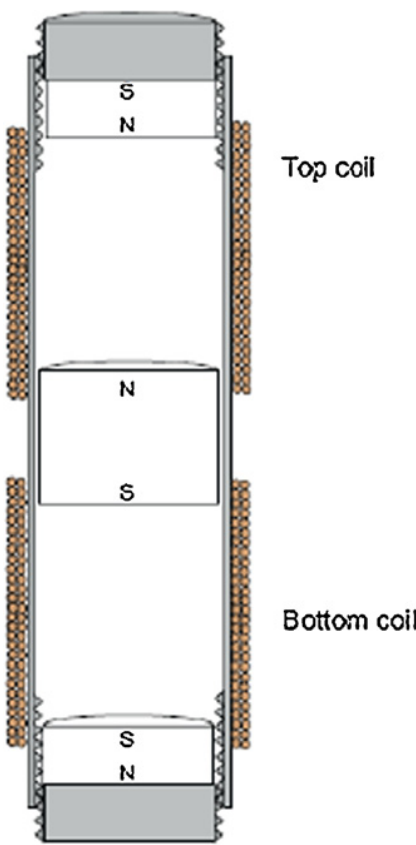

Figure 69. Schematic diagram of a magnetically levitated generator [46].

should be pointed out that the output power and bandwidth of the nonlinear generators depend on the direction of approach of the vibration frequency to the resonant frequency. For a hard nonlinearity, this approach will only produce an improvement when approaching the device resonant frequency from a lower frequency. For a soft nonlinearity, this approach will only produce an improvement when approaching the device resonant frequency from a higher frequency. It is unlikely that these conditions can be guaranteed in real application.

Nonlinear generators can be conveniently realized by using a magnetic spring instead of a conventional spring. Spreemann et al [45] reported a tunable electromagnetic vibration energy harvester with a magnetic spring, which combined a tuning mechanism with the nonlinear structure. Instead of using a linear suspension, this device was implemented using a rotary suspension (figure 67). The use of magnetic spring magnets resulted in a nonlinear restoring force. The nonlinear restoring force provides low resonance frequencies within a small generator volume and small changes in the spring magnet's position cause a significant change in the spring characteristic. As shown experimentally in figure 68 , the resonant frequency shifted by about $30 \mathrm{~Hz}$ for a displacement of $1.5 \mathrm{~mm}$ of each spring magnet. The maximum output decreased with the increase of the magnet spacing, i.e. as the resonant frequency decreased. Also the bandwidth of the device increased as the space between magnets became smaller, i.e. nonlinearity increased. This agrees with the analysis result shown in figure 66 . The generator has a volume of approximately $2.5 \mathrm{~cm}^{3}$.

In addition, the design and analysis of an energyharvesting device with magnetic restoring forces to levitate an oscillating centre magnet was presented by Mann et al [46]. Figure 69 shows the schematic diagram of the device. 


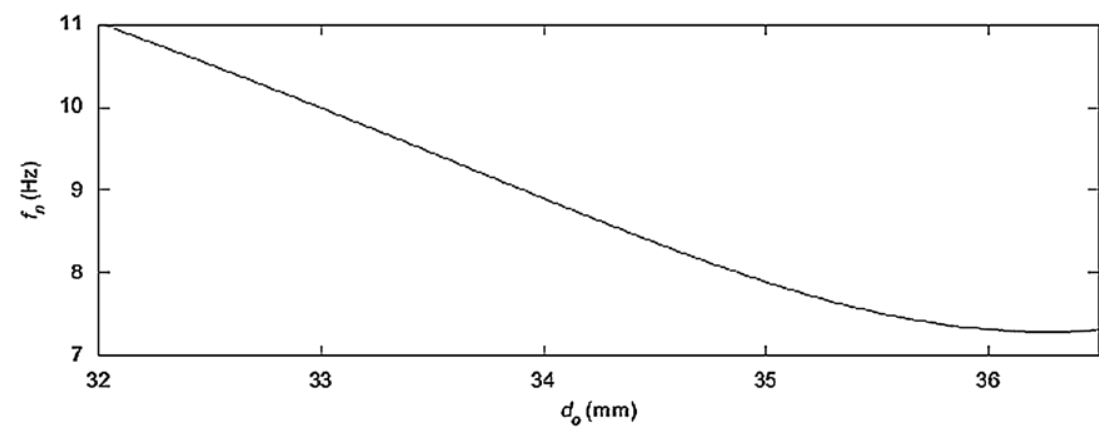

Figure 70. Change in the linear resonances as a function of the magnet spacing [46].

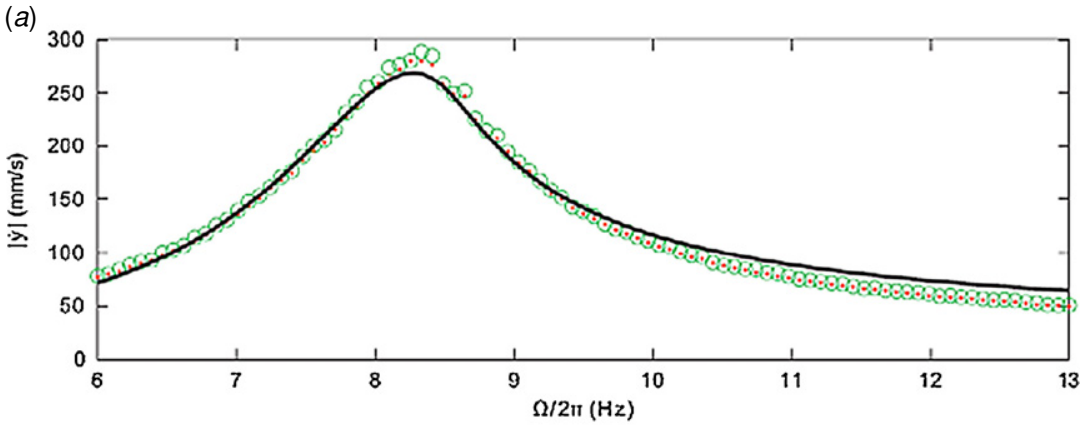

(b)

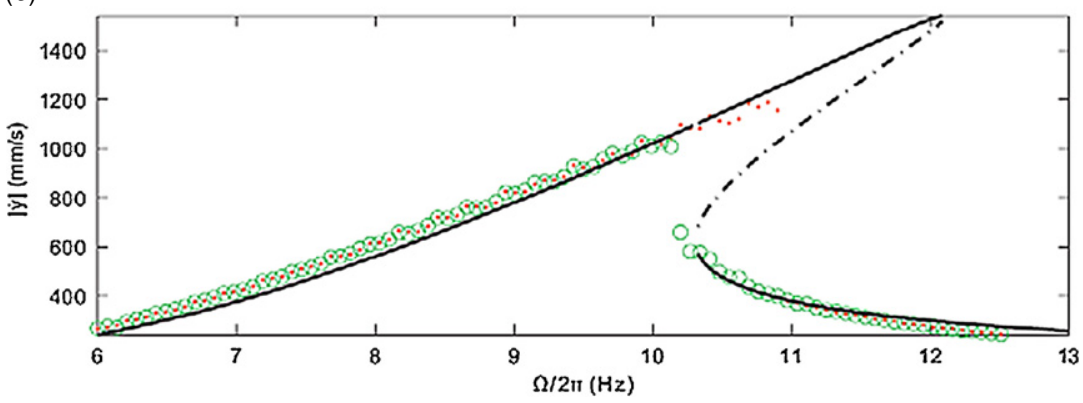

Figure 71. Experimental velocity response amplitudes from forward (dots) and reverse frequency sweeps (circles) are compared with theory. Theoretical predictions are separated into stable solutions (solid line) and unstable solutions (dashed line): (a) excitation level of $2.1 \mathrm{~m} \mathrm{~s}^{-2}$ and (b) excitation level of $8.4 \mathrm{~m} \mathrm{~s}^{-2}$ [46].

The device used two outer magnets that were mechanically attached to a threaded support. A centre magnet was placed between the two outer magnets and the magnetic poles were oriented to repel the centre magnet, thus suspending the centre magnet with a nonlinear restoring force. The nonlinearity allows the linear resonance to be tuned by simply changing the spacing between outer and centre magnets (figure 70).

The mathematical model for the energy-harvesting device was derived and examined for the case of harmonic base excitation. It was found theoretically and experimentally that the response for both linear and nonlinear systems scales almost linearly within some regimes of excitation amplitudes (figure 71(a)). However, once the nonlinearities have been sufficiently engaged, as shown in figure $71(b)$, the peak response of the nonlinear system no longer scales linearly and is relocated away from linear resonance. Thus, the ability to tune the restoring forces is an essential consideration for applications with a fixed frequency harmonic excitation so that the best performance can be obtained. Additionally, in the frequency response for the nonlinear system, relatively large amplitudes persist over a much larger range of frequencies, which could prove beneficial for applications with either fixed or varying excitation inputs. Furthermore, the maximum output power of such devices is delivered to the electrical load at a frequency away from linear resonance.

Burrow et al $[47,48]$ reported another nonlinear generator. It consisted of a linear spring with the nonlinearity caused by the addition of magnetic reluctance forces. Figure 72 shows the schematic diagram of the nonlinear generator. The flux concentrator guides the magnetic flux through the coil. The vibration of the magnets causes a change in direction of the magnetic flux, which induces a voltage across the coil. The reluctance force between the magnets and the flux concentrator resulted in the nonlinearity. Theoretically, it was found that, for a given displacement and mass, there is more energy stored in a nonlinear system than in a linear system as the force on the spring at a given peak displacement will be higher than in the linear case and the mass velocity will be higher when it passes 
Table 2. Summary of continuous tuning methods.

\begin{tabular}{llllll}
\hline Reference & $\begin{array}{l}\text { Untuned resonant } \\
\text { frequency }(\mathrm{Hz})\end{array}$ & $\begin{array}{l}\text { Tuning } \\
\text { range }(\mathrm{Hz})\end{array}$ & Size & $\begin{array}{l}\text { Tuning } \\
\text { voltage }(\mathrm{V})\end{array}$ & $\begin{array}{l}\text { Sensitivity } \\
(\mathrm{Hz} \mathrm{V}\end{array}$ \\
\hline Scheibner [15] & Multiple & $1-10 \mathrm{k}$ & $7 \times 10 \mathrm{~mm}^{2}$ & $0-35$ & 257 \\
Adams [16] & $25 \mathrm{k}$ & $1.9-36.5 \mathrm{k}$ & $500 \times 10 \mu \mathrm{m}^{2}$ & $0-50$ & 692 \\
Lee [17] & $19 \mathrm{k}$ & $5.55-19 \mathrm{k}$ & $460 \times 840 \mu \mathrm{m}^{2}$ & $0-150$ & 89.67 \\
Piazza [18] & $719 \mathrm{k}$ & $713-716 \mathrm{k}$ & $200 \times 20 \times 4.2 \mu \mathrm{m}^{3}$ & $0-20$ & 300 \\
Yao [19] & $960 \mathrm{k}$ & $900-960 \mathrm{k}$ & $\mathrm{N} / \mathrm{A}$ & $0-35$ & 1714 \\
Yao [19] & $149.5 \mathrm{k}$ & $139.5-149.5 \mathrm{k}$ & $\mathrm{N} / \mathrm{A}$ & $0-30$ & 333 \\
Peters [21] & 78 & $66-89$ & $\mathrm{~N} / \mathrm{A}$ & $-5-+5$ & 2.3 \\
Remtema [23] & $31 \mathrm{k}$ & $31-33 \mathrm{k}$ & $500 \times 700 \mu \mathrm{m}^{2}$ & $0-25 \mathrm{~mW}$ & $80 \mathrm{~Hz} \mathrm{~mW}^{-1}$ \\
Syms [24] & $\mathrm{N} / \mathrm{A}$ & $-25 \%-50 \%$ & $3000 \times 5000 \mu \mathrm{m}^{2}$ & $1.5-10 \mathrm{~mW}$ & $8.8 \% \mathrm{~mW}^{-1}$ \\
Cabuz [28] & 518 & $518-534$ & $1000 \times 200 \times 3 \mu \mathrm{m}^{3}$ & $0-16$ & 1 \\
Mukherjee [30] & $15.5 \mathrm{k}$ & $15.4-16$ & $\mathrm{~N} / \mathrm{A}$ & $0-50$ & 12 \\
\hline
\end{tabular}

Table 3. Summary of intermittent tuning methods used in vibration energy harvesting.

\begin{tabular}{|c|c|c|c|c|c|c|c|c|c|}
\hline Reference & $\begin{array}{l}\text { Untuned } \\
\text { resonant } \\
\text { frequency } \\
(\mathrm{Hz})\end{array}$ & $\begin{array}{l}\text { Tuning } \\
\text { range } \\
(\mathrm{Hz})\end{array}$ & $\begin{array}{l}\text { Mass } \\
(\mathrm{g})\end{array}$ & Size $\left(\mathrm{mm}^{3}\right)$ & $\begin{array}{l}\text { Tuning } \\
\text { force } \\
\text { (N) }\end{array}$ & $\begin{array}{l}\text { Excitation } \\
\text { level } \\
\left(\mathrm{m} \mathrm{s}^{-2}\right)\end{array}$ & $\begin{array}{l}\text { Output } \\
\text { power } \\
(\mu \mathrm{W})\end{array}$ & Sensitivity & $\begin{array}{l}\text { Affect } \\
\text { on } \\
\text { damping }\end{array}$ \\
\hline $\mathrm{Wu}$ [13] & $\mathrm{N} / \mathrm{A}$ & $130-180$ & $\mathrm{~N} / \mathrm{A}$ & $10 \times 12 \times 38^{a}$ & $\mathrm{~N} / \mathrm{A}^{\mathrm{b}}$ & 1.96 & $5-11 V_{\mathrm{oc}}$ & $1.67 \mathrm{~Hz} \mathrm{~mm}^{-1}$ & $\mathrm{~N} / \mathrm{A}$ \\
\hline Challa [22] & 26 & $22-32$ & 45.8 & $34 \times 20 \times 0.92$ & $\mathrm{~N} / \mathrm{A}^{\mathrm{c}}$ & 0.8 & $240-280$ & $0.33 \mathrm{~Hz} \mathrm{~mm}^{-1}$ & $\uparrow \mathrm{e}$ \\
\hline Leland [29] & 250 & $200-250$ & 7.1 & $\begin{array}{l}31.7 \times 12.7 \times \\
0.509\end{array}$ & $0-60$ & 9.8 & $300-400$ & $0.83 \mathrm{~Hz} \mathrm{~N}^{-1}$ & $\uparrow \mathrm{e}$ \\
\hline $\begin{array}{l}\text { Eichhorn } \\
\text { [32] }\end{array}$ & 380 & $290-380$ & $\mathrm{~N} / \mathrm{A}$ & $20 \times 5 \times 0.44$ & $0-22.75$ & 63.7 & $3-4.5 V_{\mathrm{oc}}$ & $3.96 \mathrm{~Hz} \mathrm{~N}^{-1}$ & $\uparrow \mathrm{e}$ \\
\hline Zhu [33] & 45 & 67.6-98 & $\mathrm{N} / \mathrm{A}$ & $\mathrm{N} / \mathrm{A}$ & $\mathrm{N} / \mathrm{A}^{\mathrm{d}}$ & 0.588 & $61.6-156.6$ & $8 \mathrm{~Hz} \mathrm{~mm}^{-1}$ & $-{ }^{\mathrm{f}}$ \\
\hline
\end{tabular}

a Size of the mass.

${ }^{\mathrm{b}}$ Change in position of centre of gravity: $30 \mathrm{~mm}$.

c Tuning distance: $30 \mathrm{~mm}$.

d Tuning distance: $3.8 \mathrm{~mm}$.

e Damping increased with tuning mechanism.

${ }^{\mathrm{f}}$ Damping stayed constant for most of the tuning range and increased when the tuning force became large.

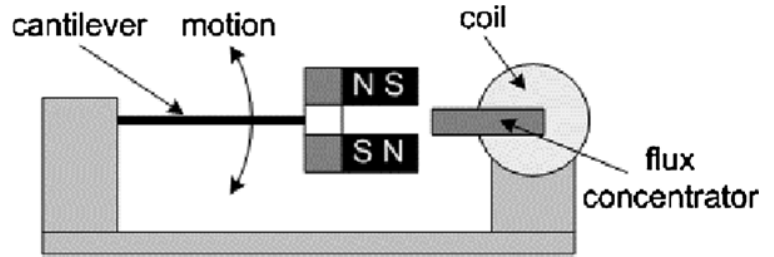

Figure 72. Schematic diagram of a nonlinear generator (after [48]).

zero displacement. In addition, the generator experimentally showed a wider bandwidth during an up-sweep, i.e. when the excitation frequency was gradually increased while the bandwidth was much narrower during a down-sweep, i.e. when the excitation frequency was gradually decreased.

\subsection{Bi-stable structures for vibration energy harvesting}

Ramlan et al [44] studied a bi-stable structure for energy harvesting (also termed the snap-through mechanism). These structures employ a negative stiffness which has the effect of steepening the displacement response of the resonator as a function of time resulting in a higher velocity for a given input excitation. Analysis revealed that the amount of power harvested by a nonlinear device is $4 / \pi$ greater than that by the
Table 4. Summary of generator arrays.

\begin{tabular}{lcll}
\hline Reference & $\begin{array}{l}\text { Tuning } \\
\text { range }(\mathrm{Hz})\end{array}$ & $\begin{array}{l}\text { Number of } \\
\text { cantilever }\end{array}$ & $\begin{array}{l}\text { Power level } \\
(\mu \mathrm{W})\end{array}$ \\
\hline Xue [39] & $92-110$ & 10 & $>30^{\mathrm{a}}$ \\
Feng [40] & $300-800$ & 4 & $>2^{\mathrm{a}}$ \\
Sari [42] & $3300-3600$ & 40 & $>0.5$ \\
\hline
\end{tabular}

${ }^{\text {a }}$ Simulation results.

tuned linear device provided the device produces a square wave output for a given sinusoidal input. Numerical results also showed that more power is harvested by the mechanism if the excitation frequency is much less than the generator's resonant frequency. Although the bi-stable mechanism cannot produce a square wave-like response under all operating conditions, it offers better performance than the linear mechanism at lower frequencies than the resonant frequency of the linear device. Bi-stable devices also have the potential to cope with the mismatch between the resonant frequency and the vibration frequency.

Dogheche et al [49] have reported a piezoelectric micromachined ultrasonic transducer (pMUT) used as a mechanical to electrical energy scavenger. The resonator was a silicon membrane of which the thickness varied from 1 to $5 \mu \mathrm{m}$ and of four different diameters, 132, 200, 400 and $600 \mu \mathrm{m}$. 
Table 5. List of specifications in figure 73.

\begin{tabular}{|c|c|c|c|c|c|c|c|}
\hline Figure & $\begin{array}{l}\text { Operational } \\
\text { frequency } \\
\text { range }\end{array}$ & $\begin{array}{l}\text { Q-factor } \\
\text { of G1 }\end{array}$ & $\begin{array}{l}\text { Q-factor } \\
\text { of } \mathrm{G} 2\end{array}$ & $\begin{array}{l}\text { Number of } \\
\text { individual } \\
\text { generator in } \\
\mathrm{G} 2, n\end{array}$ & $\begin{array}{l}\text { Resonant } \\
\text { frequencies of } \\
\text { individual } \\
\text { generators in G2 }\end{array}$ & $\begin{array}{l}\text { Q-factor } \\
\text { of G3 }\end{array}$ & $\begin{array}{l}\text { Mass } \\
\text { ratio } \\
(\mathrm{G} 1: \mathrm{G} 2: \mathrm{G} 3)\end{array}$ \\
\hline $73(a)$ & $94-106 \%$ & 10 & 100 & 9 & $91.5 \%+n \times 1.8 \%$ & 200 & $30: 15: 1$ \\
\hline $73(b)$ & $94-106 \%$ & 10 & 200 & 12 & $92.5 \%+n \times 1.2 \%$ & 200 & $30: 10: 1$ \\
\hline $73(c)$ & $55-183 \%$ & 1 & 10 & 31 & $47 \%+n \times 5 \%$ & 200 & 200:100:1 \\
\hline $73(d)$ & $55-183 \%$ & 1 & 100 & 45 & $47 \%+n \times 3 \%$ & 200 & 200:90:1 \\
\hline
\end{tabular}

Table 6. Comparisons of different strategies.

\begin{tabular}{|c|c|c|}
\hline Strategies & Advantages & Disadvantages \\
\hline Mechanical tuning & • High efficiency & $\begin{array}{l}\text { - Extra system and energy are required } \\
\text { - Responds to only one frequency at a time } \\
\text { - Slow response to a change in a vibration } \\
\text { frequency }\end{array}$ \\
\hline - Change dimension & - Does not affect damping & $\begin{array}{l}\text { - Difficult to implement } \\
\text { - Not suitable for tuning in situ }\end{array}$ \\
\hline - Change centre of gravity & - Does not affect damping & - Not suitable for tuning in situ \\
\hline $\begin{array}{l}\text { - Change spring stiffness } \\
\text { continuously }\end{array}$ & - Suitable for in situ tuning & $\begin{array}{l}\text { - Consumes energy when generators work } \\
\text { at resonance }\end{array}$ \\
\hline $\begin{array}{l}\text { - Apply axial load (change spring } \\
\text { stiffness intermittently) }\end{array}$ & $\begin{array}{l}\text { - Easy to implement } \\
\text { - Suitable for in situ tuning } \\
\text { - No energy is required when generators work at } \\
\text { resonance } \\
\text { - Damping is not affected when the tensile load is } \\
\text { applied }\end{array}$ & $\begin{array}{l}\text { - Increased damping when the } \\
\text { compressive load is applied }\end{array}$ \\
\hline Electrical tuning & $\begin{array}{l}\text { - Easy to implement } \\
\text { - No energy is required when generators work at } \\
\text { resonance } \\
\text { - Suitable for in situ tuning }\end{array}$ & - Low tuning efficiency \\
\hline Widen bandwidth & $\begin{array}{l}\text { - No tuning mechanism required } \\
\text { - Respond to different frequencies at the same } \\
\text { time } \\
\text { - Immediate response to a change in vibration } \\
\text { frequency }\end{array}$ & - Complexity in design \\
\hline - Generator array & - Damping is not affected & $\begin{array}{l}\text { - Complexity in design } \\
\text { - Low volume efficiency }\end{array}$ \\
\hline - Use mechanical stopper & - Easy to implement & $\begin{array}{l}\text { - Fatigue problem } \\
\text { - Decrease in the maximum output power }\end{array}$ \\
\hline - Coupled oscillators & - Easy to implement & - Decrease in the maximum output power \\
\hline - Nonlinear generators & $\begin{array}{l}\text { - Better performance at excitation frequencies } \\
\text { higher than resonant frequency }\end{array}$ & $\begin{array}{l}\text { - Complexity in design } \\
\text { - Hysteresis }\end{array}$ \\
\hline - Bi-stable structure & $\begin{array}{l}\text { - Better performance at excitation frequencies } \\
\text { much lower than resonant frequency }\end{array}$ & - Complexity in design \\
\hline
\end{tabular}

a Tuning while the generator is mounted on the vibration source and working.

Different pMUT devices have been tested using a hand shaking excitation ranging from 4.9 to $19.6 \mathrm{~m} \mathrm{~s}^{-2}$. The experimental results showed that the pMUT device can generate power in both linear (elastic) and nonlinear (bi-stable) mechanical behaviours.

\section{Summary}

Tables 2 and 3 summarize continuous and intermittent tuning methods. The continuous tuning devices relate to resonator devices and have not yet been applied to vibration energy harvesting. The intermittent tuning summary table contains information from actual vibration energy harvesters. Table 4 summarizes generator arrays.

\section{Comparisons of different strategies}

It has been proven theoretically and experimentally that both tuning resonant frequency and widening the bandwidth 


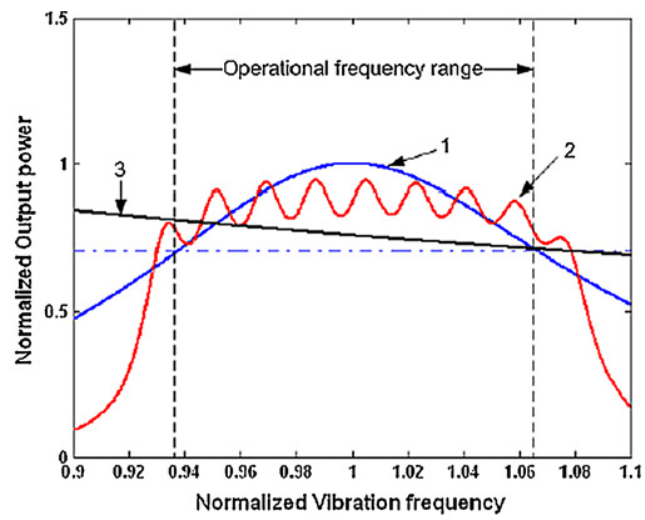

(a)

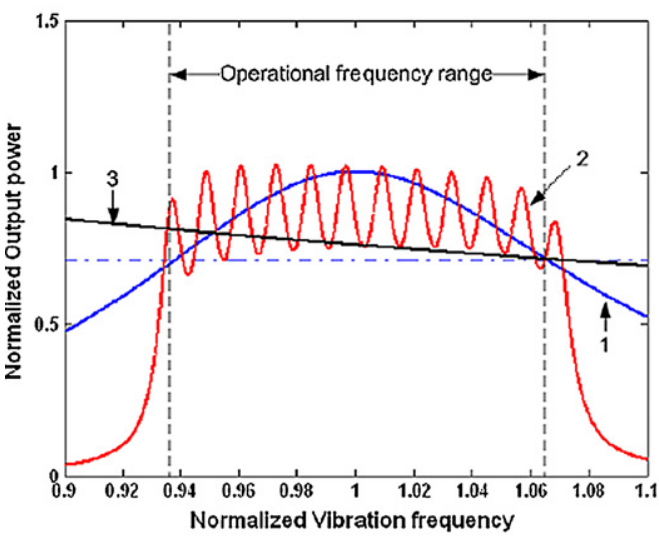

(b)

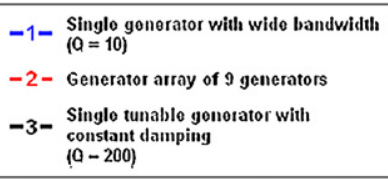

$$
\begin{aligned}
& -1-\begin{array}{l}
\text { Single generator vith wide bandwidth } \\
(Q-10)
\end{array} \\
& -2-\begin{array}{l}
\text { Generator array of } 12 \text { generators } \\
-3-
\end{array} \\
& \begin{array}{l}
\text { Single tunable generator vith } \\
\text { constant damping } \\
(Q=200)
\end{array}
\end{aligned}
$$

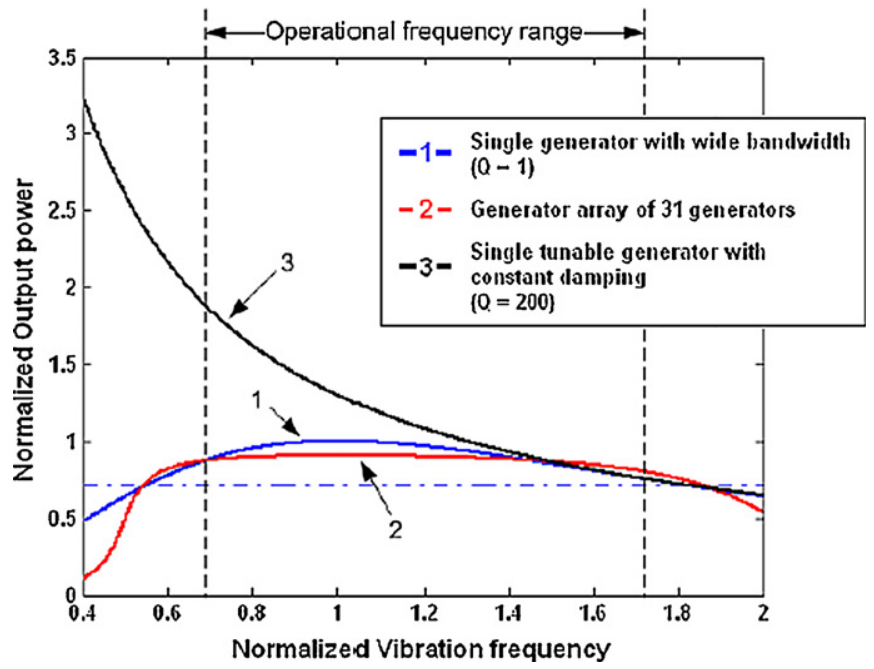

(c)

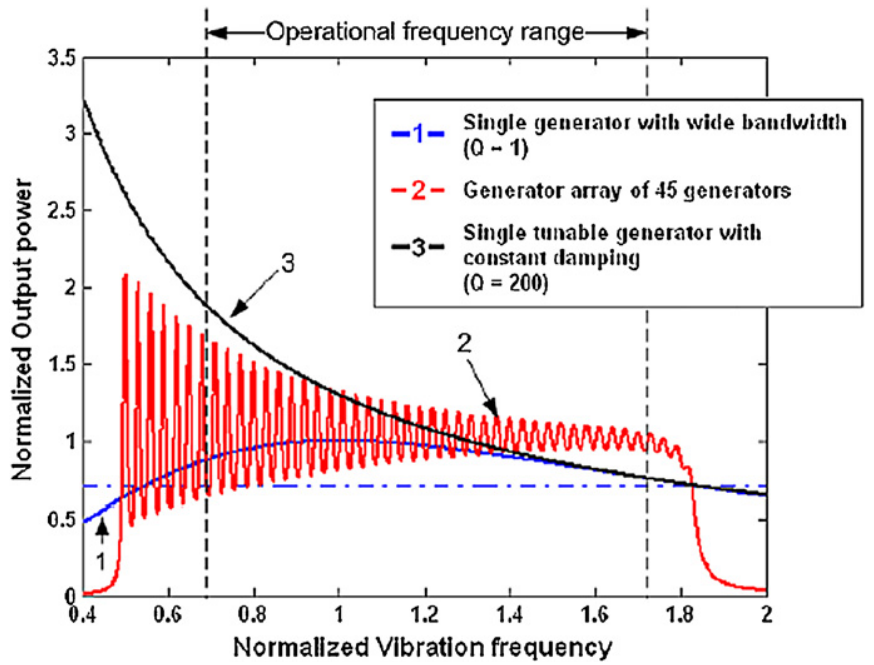

$(d)$

Figure 73. Comparison of a single generator with a wide bandwidth, a generator array and a single tunable generator with constant damping. (a) Curves 1 and 3 are both single generators. Curve 2 consists of a generator array of nine generators of a Q-factor of 100 . (b) Curves 1 and 3 are identical to $(a)$. Curve 2 now consists of a generator array of 12 generators of a Q-factor of 200. (c) For curve 1, the Q-factor has been reduced to 1 . Curve 2 shows a generator array of 31 generators of a Q-factor of 10 . Curve 3 has the same Q-factor as in $(a)$ and $(b)$ but with higher mass. $(d)$ Curves 1 and 3 are identical to $(c)$. Curve 2 now consists of a generator array of 45 generators of a Q-factor of 100 .

of vibration-based micro-generators can increase their operational frequency range.

To compare the performance of a single generator with a wide bandwidth, a generator array and a single tunable generator with constant damping, typical specifications of these three types of generators have been chosen. G1, G2 and
G3, listed in table 5, represent a single generator with a wide bandwidth, a generator array and a single tunable generator with constant damping, respectively. Figure 73 shows the comparison of power spectra of these three types of generator.

When the Q-factor of a single generator decreases, its bandwidth increases. To generate the same amount of output 
power as the original bandwidth, a single generator has to be larger as the bandwidth increases.

If a generator array is used to widen the operational frequency range, one can design a few larger individual generators with low Q-factor with a large resonant frequency gap between generators or many smaller individual generators with high Q-factor but a small resonant frequency gap between generators. By contrast, it is much easier to design small tunable generators with constant damping to cover the same amount of operational frequency range at the cost of extra energy to power the frequency tuning mechanisms.

Table 6 compares the advantages and disadvantage of different strategies to increase the operational frequency range of vibration-based micro-generators.

\section{Conclusions}

As most practical applications for vibration-based microgenerators exhibit frequency variations over time, it is not possible to guarantee that fixed frequency generators will always work at resonance and produce the maximum output power. Mechanisms have to be employed to increase the operational frequency range of vibration-based microgenerators. There are two possible solutions, i.e. tuning the resonant frequency of a single generator and widening the bandwidth of the generator. Practical realizations of each solution and their characteristics are summarized in this section.

\subsection{Tuning the resonant frequency of a single generator}

The first solution, i.e. tuning the resonant frequency of a single generator, requires a certain mechanism to periodically adjust the resonant frequency so that it matches the frequency of ambient vibration at all times; maximum power can then be generated at various frequencies without reducing the Q-factor and with high efficiency per unit volume. Intermittent tuning has advantages over continuous tuning as it is more efficient because the tuning mechanism is turned off when the generator is at the right frequency thereby consuming negligible energy, which makes producing a net output power more probable.

Among mechanical methods of frequency tuning, changing the dimensions of the structure and the position of the centre of gravity is potentially suitable for intermittent tuning. However, it is less suitable for continuous tuning since it is problematic to change and maintain the new dimensions of the structure or the centre of gravity of the proof mass during operation. The most suitable approach to changing the dimensions of the structure is to change its length. This requires the structure clamp is removed, the length adjusted and then the structure re-clamped. It is important that in each tuning procedure the structure is clamped properly; otherwise the performance of the generator will be severely affected by introducing damping effects through the supports. Therefore, these two methods are not suitable for in situ tuning (tuning while the generator is mounted on the vibration source and working) or tuning with automatic control.

Alternatively the frequency can be tuned by changing the spring stiffness intermittently or continuously. They are both suitable for in situ tuning, but intermittently changing the spring stiffness is always preferred for efficiency reasons. However, extra systems and energy are required to realize tuning using mechanical methods.

It is important to mention that the efficiency of mechanical tuning methods also depends on the size of the structure. The smaller the resonator, the higher the efficiency of the tuning mechanism.

Resonant frequency tuning by adjusting the electrical load has been practically shown to be feasible. This method consumes little energy as it does not involve any change in mechanical properties. The only energy consumed is in the electronic switches and control unit, which is typically far less than that consumed in mechanical tuning methods. In addition, it is much easier to implement than mechanical methods. However, the tuning efficiency to date is quite low and this method cannot achieve a large tuning range. An extra closed loop system also has to be introduced to control the tuning process.

\subsection{Widening the bandwidth of the generator}

For the second solution, i.e. to widen the bandwidth, an obvious problem is that there is a trade-off between the system bandwidth and the Q-factor. A wider bandwidth means, for a single resonator, a lower Q-factor, which reduces the maximum output power. A bandwidth can also be effectively widened by designing a generator consisting of an array of small generators, each of which works at a different frequency. Thus, the assembled generator has a wide operational frequency range whilst the Q-factor does not decrease. However, this assembled generator must be carefully designed so that each individual generator does not affect the others. This makes it more complex to design and fabricate. Additionally, at a particular source frequency, only a single or a few individual generators contribute to the power output so the approach is volume inefficient.

Another method used to increase the bandwidth is to use an amplitude limiter to limit the amplitude of the resonator. The drawbacks are that this method causes the maximum output power to drop by limiting the vibration amplitude and the repeating mechanical contact between the cantilever and the mechanical stopper may result in earlier fatigue-induced failure in the cantilever beam.

Using coupled oscillators can also increase the operational bandwidth of the generator. It can achieve flat response over a wide frequency range. However, the maximum output power of a coupled oscillator generator is significantly lower than that of a generator with a single mass.

Furthermore, nonlinear generators and generators with bi-stable structures are two further potential solutions to increase the operational frequency range of vibration-based micro-generators. They can improve the performance of the generator at higher and lower frequency bands relative to its resonant frequency, respectively. However, the mathematical modelling of these generators is more complicated than that of linear generators, which increases the complexity in design and implementation. Besides, there is hysteresis in nonlinear 


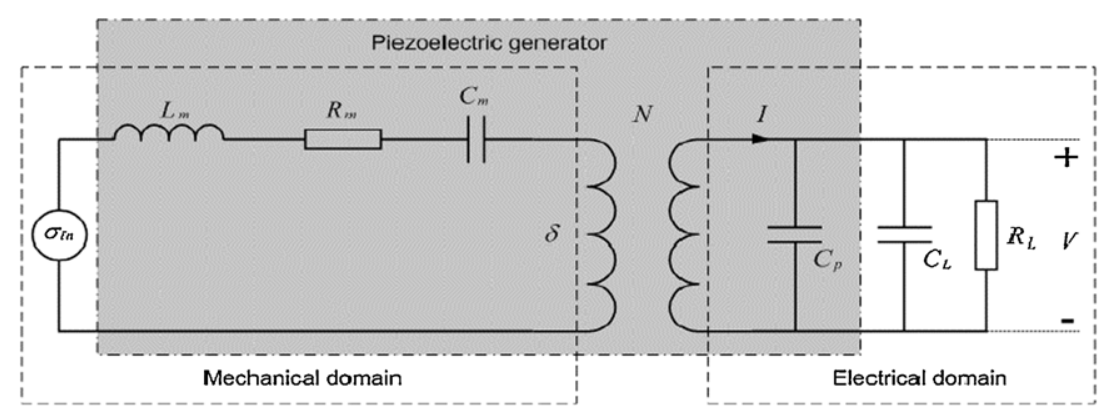

Figure A1. Equivalent circuit of a piezoelectric generator with capacitive and resistive loads.

generators. Performance during a down-sweep (or an upsweep) can be worse than that during an up-sweep (or a downsweep) or worse than the linear region depending on the sweep direction as explained in section 5.3.

In conclusion, for vibration energy harvesting, possible strategies to increase the operation frequency range include the following:

- changing spring stiffness intermittently (preferred) or continuously,

- straining the structure intermittently (preferred) or continuously,

- adjusting capacitive load,

- using generator array,

- employing nonlinear and bi-stable structures.

To realize these strategies properly, the following issues have to be considered. For intermittent mechanical tuning, the tuning system has to be designed to consume as little energy as possible and not to affect the damping so as to make the generator harvest the maximum power. In addition, currently commercially available linear actuators are still large in size compared to mm-scale micro-generator. To keep tunable generators of reasonable size, it is important to use miniature actuators. Generators using electrical tuning must be well designed in order to achieve sufficient coupling between the mechanical and electrical domains to enable larger tuning ranges. Moreover, nonlinear generators and generators with bi-stable structures have not been sufficiently developed and further attention should be paid to practically implement them.

The last decade has seen great improvement of vibrationbased micro-generators in powering wireless sensor networks by continuous effort of research groups and companies all around the world. The development of strategies to increase the operational frequency range of vibration-based microgenerators will bring these energy sources to much wider application.

\section{Appendix}

The bimorph piezoelectric generators as shown in figure 46 can be represented using an equivalent circuit as shown in figure A1. $L_{m}, R_{m}$ and $C_{m}$ represent the mass, damping and spring in the mechanical part, respectively. $C_{p}$ is the capacitance of the piezoelectric layer, and $C_{L}$ and $R_{L}$ are the capacitive and resistive load, respectively. $V$ is the RMS voltage across the resistive load.
The transformer relates the mechanical domain to the electrical domain according to the model of the piezoelectric effect. Specifically, it relates the stress $(\sigma)$ to the electric field $(E)$ at zero strain, or the electrical displacement $(D)$ to the strain $(\delta)$ at zero electric field. The equations for the piezoelectric effect are

$$
\begin{aligned}
& \delta=\frac{\sigma}{Y_{p}}+d E \\
& D=\varepsilon E+d \sigma
\end{aligned}
$$

where $d$ is the piezoelectric strain coefficient, which is $d_{31}$ for a piezoelectric bimorph as it works in 31 mode, $\varepsilon$ is the dielectric constant of the piezoelectric material and $Y_{p}$ is Young's modulus of the piezoelectric material. Rewriting (A.1) and (A.2) leads to the equations for the transformer as

$$
D=-d_{31} Y_{p} \delta \text {. }
$$

Hence, the transform ratio $N$ is given by

$$
N=-d_{31} Y \text {. }
$$

Equation (A.5) can be derived to present the mechanical dynamics of the system with electrical coupling. Detailed derivation of this model can be found in [50]:

$$
\Delta\left(s^{2}+2 \zeta \omega_{n} s+\omega_{n}^{2}\right)=\frac{\omega_{n}^{2} d_{31} a}{2 t_{c}} V+b^{*} A_{\text {in }}
$$

where $\Delta$ is the Laplace transform of strain, $\delta, A_{\text {in }}$ is the vibration acceleration, $\zeta$ is the damping factor, $\omega_{n}$ is the untuned resonant frequency, $a=1$ if the two piezoelectric layers are connected in series and $a=2$ if they are connected in parallel and $s$ is the Laplace variable. $b^{*}$ is given by

$$
b^{*}=\frac{3 t_{g}}{l_{b}^{2}} \cdot \frac{\left(2 l_{b}+l_{m}-l_{e}\right)}{\left(2 l_{b}+\frac{3}{2} l_{m}\right)}
$$

where $l_{e}$ is the length of the electrodes.

Furthermore, analysis in the electrical domain gives the following equation:

$$
\Delta=\left(s+\frac{1}{R_{L} C_{p L}}\right) \cdot \frac{V \cdot C_{p L}}{s \cdot \Sigma}
$$

where $C_{p L}=C_{p}+C_{L}$ and $\Sigma=-a \cdot d_{31} \cdot Y_{c} \cdot l_{e} \cdot w . l_{e}$ is the length of the electrodes.

Combining equations (A.5) and (A.7) gives the transfer function of the system as

$$
\frac{V}{A_{\text {in }}}=\frac{s \cdot \Sigma \cdot b^{*}}{s^{3}+\left(\frac{1}{R_{L} C_{p L}}+2 \zeta \omega_{n}\right) s^{2}+\left(\omega_{n}^{2}+\frac{2 \zeta \omega_{n}}{R_{L} C_{p L}}-\frac{a \Sigma \omega_{n}^{2} d_{31}}{2 t_{c}}\right) s+\frac{\omega_{n}^{2}}{R_{L} C_{p L}}}
$$


which leads to the expression of the voltage across the resistive load given by

$V(\omega)=\frac{\mathrm{j} \omega \cdot \Sigma \cdot b^{*} \cdot A_{\text {in }}}{\left[\frac{\omega_{n}^{2}}{R_{L}}-\left(\frac{1}{R_{L}}+2 \zeta \omega_{n} C_{p L}\right) \omega^{2}\right]+\mathrm{j} \omega\left(\left(\omega_{n}^{2}-\omega^{2}\right) C_{p L}+\frac{2 \zeta \omega_{n}}{R_{L}}-\frac{a \Sigma \cdot \omega_{n}^{2} d_{31}}{2 t_{c}}\right)}$.

The power in the resistive load is given by

$$
\begin{aligned}
& P(\omega)=\frac{V(\omega)^{2}}{R_{L}} \\
& =\frac{1}{R_{L}} \cdot \frac{\left(\omega \cdot \Sigma \cdot b^{*} \cdot A_{\text {in }}\right)^{2}}{\left[\frac{\omega_{n}^{2}}{R_{L}}-\left(\frac{1}{R_{L}}+2 \zeta \omega_{n} C_{p L}\right) \omega^{2}\right]^{2}+\omega^{2}\left(\left(\omega_{n}^{2}-\omega^{2}\right) C_{p L}+\frac{2 \zeta \omega_{n}}{R_{L}}-\frac{a \Sigma \cdot \omega_{n}^{2} d_{31}}{2 t_{C}}\right)^{2}} .
\end{aligned}
$$

It is known that $f(x, y)=x^{2}+y^{2} \geqslant 2 x y$ and that $f(x, y)$ becomes a minimum only if $x=y$ (i.e. $\frac{1}{f(x, y)}$ is maximum only if $x=y$ ). Therefore, (A.10) reaches the maximum when

$$
\begin{aligned}
& \frac{\omega_{n}^{2}}{R_{L}}-\left(\frac{1}{R_{L}}+2 \zeta \omega_{n} C_{p L}\right) \omega^{2} \\
& =\omega\left(\left(\omega_{n}^{2}-\omega^{2}\right) C_{p L}+\frac{2 \zeta \omega_{n}}{R_{L}}-\frac{a \Sigma \cdot \omega_{n}^{2} d_{31}}{2 t_{c}}\right) .
\end{aligned}
$$

Rearranging (A.11) leads to a cubic function of the form

$$
\omega^{3}+X \omega^{2}+Y \omega+Z=0
$$

where

$$
\begin{gathered}
X=-\left(\frac{1}{R_{L} C_{p L}}+2 \zeta \omega_{n}\right) \\
Y=-\left(\omega_{n}^{2}+\frac{2 \zeta \omega_{n}}{R_{L} C_{p L}}-\frac{a \Sigma \cdot \omega_{n}^{2} d_{31}}{2 t_{c} C_{p L}}\right) \\
Z=\frac{\omega_{n}^{2}}{R_{L} C_{p L}} .
\end{gathered}
$$

The real solution of (A.12) gives the function of a resonant frequency with respect to the load capacitance as

$$
\omega\left(C_{L}\right)=\frac{\sqrt[3]{\Omega+12 \sqrt{\Psi}}}{6}-\frac{2\left(Y-\frac{X^{2}}{3}\right)}{\sqrt[3]{\Omega+12 \sqrt{\Psi}}}-\frac{X}{3}
$$

where

$$
\begin{gathered}
\Omega=36 X Y-108 Z-8 X^{3} \\
\Psi=12 Y^{3}-3 X^{2} Y^{2}-54 X Y Z+81 Z^{2}+12 X^{3} Z .
\end{gathered}
$$

Equations (A.10) and (A.13) indicate that output power and the resonant frequency of a bimorph piezoelectric generator vary with variations of the load capacitance.

\section{References}

[1] Beeby S P, Tudor M J and White N M 2006 Energy harvesting vibration sources for microsystems applications Meas. Sci. Technol. 17 R175-95

[2] Shenck N S and Paradiso J A 2001 Energy scavenging with shoe-mounted piezoelectrics IEEE Micro 21 30-42

[3] Donelan J M et al 2008 Biomechanical energy harvesting: generating electricity during walking with minimal user effort Science 319 807-10

[4] Feenstra J, Granstrom J and Sodano H 2008 Energy harvesting through a backpack employing a mechanically amplified piezoelectric stack Mech. Syst. Signal Process. 22 721-34
[5] Roundy S and Zhang Y 2005 Toward self-tuning adaptive vibration based micro-generators Proc. SPIE 5649 373-84

[6] Williams C B and Yates R B 1996 Analysis of a micro-electric generator for microsystems Sensors Actuators A 52 8-11

[7] Arnold D P 2007 Review of microscale magnetic power generation IEEE Trans. Magn. 43 3940-51

[8] Sodano H A, Inman D J and Park G 2004 A review of power harvesting from vibration using piezoelectric materials Shock Vib. Dig. 36 197-205

[9] Roundy S, Wright P and Pister K 2002 Micro-electrostatic vibration-to-electricity converters Proc. IMECE 2002 pp 1-10

[10] Blevins R D 2001 Formulas for Natural Frequency and Mode Shape (Malabar, FL: Krieger) p 158

[11] Gieras J F, Oh J-H, Huzmezan M and Sane H S 2007 Electromechanical energy harvesting system Patent Publication Number: WO2007070022(A2), WO2007070022(A3)

[12] Roylance L and Angell J B 1979 A batch fabricated silicon accelerometer IEEE Trans. Electron Devices 26 1911-7

[13] Wu X, Lin J, Kato S, Zhang K, Ren T and Liu L 2008 A frequency adjustable vibration energy harvester Proc. PowerMEMS 2008+ microEMS2008 (9-12 November 2008, Sendai, Japan) pp 245-8

[14] Scheibner D, Mehner J, Reuter D, Kotarsky U, Gessner T and Dötzel W 2004 Characterization and self-test of electrostatically tunable resonators for frequency selective vibration measurements Sensors Actuators A 111 93-9

[15] Scheibner D, Mehner J, Reuter D, Gessner T and Dötzel W 2005 A spectral vibration detection system based on tunable micromechanical resonators Sensors Actuators A 123-124 $63-72$

[16] Adams S G, Bertscht F M, Shawt K A, Hartwell P G, MacDonald N C and Moon F C 1995 Capacitance based tunable micromechanical resonators 8 th Int. Conf. on Solid-State Sensors and Actuators, and Eurosensors IX (Stockholm, Sweden) pp 438-41

[17] Lee K B, Lin L and Cho Y H 2008 A closed-form approach for frequency tunable comb resonators with curved finger contour Sensors Actuators A 141 523-9

[18] Piazza G, Abdolvand R, Ho G K and Ayazi F 2004 Voltage-tunable piezoelectrically transduced single-crystal silicon micromechanical resonators Sensors Actuators A $11171-8$

[19] Yao J J and MacDonald N C 1995 A micromachined, single-crystal silicon, tunable resonator J. Micromech. Microeng. 5 257-64

[20] Thiesen J and O'Brian G P 2006 Energy harvester with adjustable resonant frequency Patent Publication Number: WO2006046937(A1), EP1803170(A1), US2008129147(A1), EP1803170(A0), CN101002343(A)

[21] Peters C, Maurath D, Schock W and Manoli Y 2008 Novel electrically tunable mechanical resonator for energy harvesting Proc. PowerMEMS 2008+ microEMS2008, (Sendai, Japan, 9-12 November 2008) pp 253-6

[22] Challa V R, Prasad M G, Shi Y and Fisher F T 2008 A vibration energy harvesting device with bidirectional resonance frequency tunability Smart Mater. Struct. 17015035

[23] Remtema T and Lin L 2001 Active frequency tuning for micro resonators by localized thermal stressing effects Sensors Actuators A 91 326-32

[24] Syms R R A 1998 Electrothermal frequency tuning of folded and coupled vibrating micromechanical resonators $J$. Microelectromech. Syst. 7 164-71

[25] Blevins R D 2001 Formulas for Natural Frequency and Mode Shape (Malabar, FL: Krieger) p 144

[26] Blevins R D 2001 Formulas for Natural Frequency and Mode Shape (Malabar, FL: Krieger) p 109 
[27] Blevins R D 2001 Formulas for Natural Frequency and Mode Shape (Malabar, FL: Krieger) p 149

[28] Cabuz C, Fukatsu K, Hashimoto H, Shoji S, Kurabayashi T, Minami K and Esashi M 1994 Fine frequency tuning in resonant sensors Proc. IEEE Workshop on Micro Electro Mechanical Systems (Oisa, Japan, 25-28 January 1994) pp 245-50

[29] Leland E S and Wright P K 2006 Resonance tuning of piezoelectric vibration energy scavenging generators using compressive axial preload Smart Mater. Struct. 15 1413-20

[30] Mukherjee R 2007 MEMS resonator using frequency tuning Patent Publication Number: US20070214890A1

[31] $\mathrm{Hu}$ Y, Xue $\mathrm{H}$ and $\mathrm{Hu} \mathrm{H} 2007$ A piezoelectric power harvester with adjustable frequency through axial preloads Smart Mater. Struct. 16 1961-6

[32] Eichhorn C, Goldschmidtboeing F and Woias P 2008 A frequency piezoelectric energy convert based on a cantilever beam Proc. PowerMEMS 2008+ microEMS2008 (9-12 November 2008, Sendai, Japan) pp 309-12

[33] Zhu D, Roberts S, Tudor M J and Beeby S P 2008 Closed loop frequency tuning of a vibration-based micro-generator Proc. PowerMEMS 2008+ microEMS2008 (Sendai, Japan, 9-12 November 2008) pp 229-32

[34] http://www.memsnet.org/material/

[35] Wu W-J, Chen Y-Y, Lee B-S, He J-J and Peng Y-T 2006 Tunable resonant frequency power harvesting devices Proc. SPIE $616955-62$

[36] Charnegie D 2007 Frequency tuning concepts for piezoelectric cantilever beams and plates for energy harvesting $M S C$ Dissertation School of Engineering, University of Pittsburgh, USA

[37] Shahruz S M 2006 Design of mechanical band-pass filters for energy scavenging $J$. Sound Vib. 292 987-98

[38] Xue H, Hu Y and Wang Q-M 2008 Broadband piezoelectric energy harvesting devices using multiple bimorphs with different operating frequencies IEEE Trans. Ultrason., Ferroelectr. Freq. Control $552104-8$

[39] Feng G-H and Hung J-C 2007 Optimal FOM designed piezoelectric microgenerator with energy harvesting in a wide vibration bandwidth Proc. 2nd IEEE Int. Conf. on
Nano/Micro Engineered and Molecular Systems (16-19 January 2007, Bangkok, Thailand) pp 511-4

[40] Ferrari M, Ferrari V, Guizzetti M, Marioli D and Taroni A 2008 Piezoelectric multifrequency energy converter for power harvesting in autonomous microsystems Sensors Actuators A 142 329-35

[41] Sari I, Balkan T and Kulah H 2007 A wideband electromagnetic micro power generator for wireless microsystems Int. Solid-State Sensors, Actuators and Microsystems Conf. (Lyon, France) pp 275-8

[42] Soliman M S M, Abdel-Rahman E M, El-Saadany E E and Mansour R R 2008 A wideband vibration-based energy harvester J. Micromech. Microeng. 18115021

[43] Petropoulos T, Yeatman E M and Mitcheson P D 2004 MEMS coupled resonators for power generation and sensing Micromechanics Europe (5-7 September 2004, Leuven, Belgium)

[44] Ramlan R, Brennan M J, Mace B R and Kovacic I 2008 An investigation into the benefits of using a nonlinear stiffness in an energy harvesting device (submitted)

[45] Spreemann D, Folkmer B, Maurath D and Manoli Y 2006 Tunable transducer for low frequency vibrational energy scavenging Proc. EurosensorsXX (Göteborg, Sweden)

[46] Mann B P and Sims N D 2009 Energy harvesting from the nonlinear oscillations of magnetic levitation J. Sound Vib. $319515-30$

[47] Burrow S G and Clare L R 2007 A resonant generator with non-linear compliance for energy harvesting in high vibrational environments IEEE Int. Electric Machines \& Drives Conference, IEMDC '07 vol 1 pp 715-20

[48] Burrow S G, Clare L R, Carrella A and Barton D 2008 Vibration energy harvesters with non-linear compliance Proc. SPIE 6928692807

[49] Dogheche K, Cavallier B, Delobelle P, Hirsinger L, Cattan E, Rèmiens D, Marzencki M, Charlot B, Basrour S and Ballandras S $2006 \mathrm{~A}$ bi-stable micro-machined piezoelectric transducer for mechanical to electrical energy transformation Integr. Ferroelectr. 80 305-15

[50] Roundy S, Wright P K and Rabaey J M 2004 Energy Scavenging for Wireless Sensor Networks with Special Focus on Vibrations (Dordrecht: Kluwer) pp 181-8 